\title{
ON THE EXISTENCE, UNIQUENESS, AND FINITE ELEMENT APPROXIMATION OF SOLUTIONS OF THE EQUATIONS OF STATIONARY, INCOMPRESSIBLE MAGNETOHYDRODYNAMICS
}

\author{
MAX D. GUNZBURGER, AMNON J. MEIR, AND JANET S. PETERSON
}

\begin{abstract}
We consider the equations of stationary, incompressible magnetohydrodynamics posed in a bounded domain in three dimensions and treat the full, coupled system of equations with inhomogeneous boundary conditions. Under certain conditions on the data, we show that the existence and uniqueness of the solution of a weak formulation of the equations can be guaranteed. We discuss a finite element discretization of the equations and prove an optimal estimate for the error of the approximate solution.
\end{abstract}

\section{INTRODUCTION}

In this work we study the equations of stationary, incompressible magnetchydrodynamics which describe the steady state flow of a viscous, incompressible, electrically conducting fluid. There is interest in these equations since they have applications in fusion technology and novel submarine propulsion devices; they also model the flow of liquid metals in magnetic pumps that are used to cool nuclear reactors (see $[10,15,18-21])$. The majority of the work done on these equations has been for the time-dependent problem with homogeneous boundary conditions. Those studies done for the steady state problem usually treat homogeneous boundary conditions, except in the few cases where simplified and unrealistic nonhomogeneous boundary conditions are treated; see [2]. In addition, many previous studies only consider one- or two-dimensional domains, where many effects unique to physical, three-dimensional space are lost; see [21]. Also, many studies assume other simplifications to the equations, such as vanishing magnetic Reynolds number, which allows the fluid and magnetic equations to uncouple; see [14].

In this work we treat the full, coupled system of equations in a three-dimensional domain. These equations are specified in $\S 2$ along with the definition of various function spaces that will be needed. In $\S 3$ we give a weak formulation

Received August 7, 1989; revised May 25, 1990.

1980 Mathematics Subject Classification (1985 Revision). Primary 76W05; Secondary 35Q20, $65 \mathrm{~N} 15,65 \mathrm{~N} 30$.

The research of the first and second authors was partially supported by the Air Force Office of Scientific Research under Grant AFOSR-88-0197. This work was partially performed under the auspices of the U.S. Department of Energy while the authors were at Los Alamos National Laboratory. 
for these equations and in $\S 4$ we prove an existence and uniqueness result. An alternate choice of boundary conditions is discussed in $\S 5$. Section 6 deals with various aspects of discretizing the equations by finite element methods. We prove an existence and uniqueness result for the solution of the discrete equations and derive error estimates for the discrete solution. In $\S 7$ we describe three possible iterative schemes for solving the nonlinear algebraic equations which result from discretization. Our work is in the spirit of some of the wellknown analyses done for the Navier-Stokes equations; e.g., see $[6,7,9,17]$.

\section{EQUATIONS AND FUNCTION SPACES}

2.1. Equations and boundary conditions. We consider the equations of stationary, incompressible magnetohydrodynamics in a bounded domain in threedimensional space. Let $\Omega$ be a simply-connected, bounded domain in $\mathbb{R}^{3}$ which is either convex or has a $C^{1,1}$ boundary $\partial \Omega$; see [8]. We assume these restrictions on the domain $\Omega$ throughout this discussion. Consider the following equations which hold in $\Omega$ :

$$
\begin{gathered}
-\frac{1}{M^{2}} \Delta \mathbf{u}+\frac{1}{N} \mathbf{u} \cdot \nabla \mathbf{u}+\nabla p-\mathbf{j} \times \mathbf{B}=\mathbf{f}, \\
\nabla \cdot \mathbf{u}=0, \\
\mathbf{j}=\mathbf{E}+\mathbf{u} \times \mathbf{B}, \\
\nabla \cdot \mathbf{j}=0, \\
\nabla \times \mathbf{B}=R_{m} \mathbf{j}, \\
\nabla \cdot \mathbf{B}=0, \\
\nabla \times \mathbf{E}=0 .
\end{gathered}
$$

Here $M, N$, and $R_{m}$ are the Hartman number, interaction parameter, and magnetic Reynolds number, respectively, and are given by

$$
M=\bar{B} \bar{L}\left(\frac{\sigma}{\eta}\right)^{1 / 2}, \quad N=\sigma \bar{B}^{2} \frac{\bar{L}}{\rho \bar{u}}, \quad R_{m}=\mu \sigma \bar{u} \bar{L},
$$

where $\bar{u}, \bar{B}$, and $\bar{L}$ are a characteristic velocity, magnetic field, and length, respectively; see $[18,19]$. The unknowns have been nondimensionalized as follows: the velocity $\mathbf{u}$ by $\bar{u}$, the pressure $p$ by $\sigma \bar{u} \bar{B}^{2} \bar{L}$, the electric current density $\mathbf{j}$ by $\sigma \bar{u} \bar{B}$, the magnetic field $\mathbf{B}$ by $\bar{B}$, the electric field $\mathbf{E}$ by $\bar{u} \bar{B}$, and the body force $f$ by $\sigma \bar{u} \bar{B}^{2}$. The other parameters appearing above are the density $\rho$, the electrical conductivity $\sigma$, the viscosity $\eta$, and the magnetic permeability $\mu$. We assume that the scalars $\rho, \sigma, \eta$, and $\mu$ remain constant in the fluid and that these, as well as the vector $\mathbf{f}$, are given. The unknown variables are the vectors $\mathbf{u}, \mathbf{j}, \mathbf{B}, \mathbf{E}$ and the scalar $p$.

We require the solution of $(2.1)-(2.7)$ to satisfy the following boundary conditions. For the velocity we specify

$$
\left.\mathbf{u}\right|_{\partial \Omega}=\mathbf{g}
$$


where $\mathbf{g}=\mathbf{0}$ if there is no flow through the boundaries and a no-slip condition is satisfied at the boundaries. For the magnetic field we specify

$$
\left.(\mathbf{B} \cdot \mathbf{n})\right|_{\partial \Omega}=q,
$$

where $q=0$ for a perfect conducting boundary and $\mathbf{n}$ is the outward-pointing normal to $\Omega$. Lastly, we impose

$$
\left.(\mathbf{E} \times \mathbf{n})\right|_{\partial \Omega}=\mathbf{k},
$$

where $\mathbf{k}=-\left(\mathbf{u}_{b} \times \mathbf{B}\right) \times\left.\mathbf{n}\right|_{\partial \Omega}$ for a perfectly conducting boundary, where $\mathbf{u}_{b}$ denotes the velocity of the boundary; if $\mathbf{u}_{b}=0$, the boundary is fixed, and then $\mathbf{k}=0$ for a perfectly conducting boundary.

Instead of the boundary conditions (2.9) and (2.10), we can also consider the problem with the alternative boundary conditions

$$
\left.(\mathbf{B} \times \mathbf{n})\right|_{\partial \Omega}=\mathbf{q}
$$

and

$$
\left.(\mathbf{E} \cdot \mathbf{n})\right|_{\partial \Omega}=k,
$$

where we need impose condition (2.12) only when solving for $\mathbf{E}$. A derivation of these equations and boundary conditions can be found in [10, 11, 15].

2.2. Function spaces. In this subsection we introduce some function spaces and their associated norms, along with some related notation; see $[1,4,7,8]$. We define the Sobolev spaces $H^{m}(\Omega)$ for nonnegative integers $m$ by

$$
H^{m}(\Omega):=\left\{w \in L^{2}(\Omega): \mathbf{D}^{\gamma} w \in L^{2}(\Omega) \forall \gamma:|\gamma| \leq m\right\},
$$

where $\gamma=\left(\gamma_{1}, \gamma_{2}, \gamma_{3}\right)$ is a multi-index and $|\gamma|=\gamma_{1}+\gamma_{2}+\gamma_{3}$. Clearly, $H^{0}(\Omega)=$ $L^{2}(\Omega)$. The norm associated with $H^{m}(\Omega)$ that we will use is

$$
\|w\|_{m}:=\left(\sum_{|\gamma|=0}^{m}\left\|\mathbf{D}^{\gamma} w\right\|_{0}^{2}\right)^{1 / 2} .
$$

For vector-valued functions we define the Sobolev space $\mathbf{H}^{m}(\Omega)$ by

$$
\mathbf{H}^{m}(\Omega):=\left\{\mathbf{w}: w_{i} \in H^{m}(\Omega), i=1,2,3\right\},
$$

where $\mathbf{w}=\left(w_{1}, w_{2}, w_{3}\right)$, and its associated norm $\|\cdot\|_{m}$ by

$$
\|\mathbf{w}\|_{m}=\left(\sum_{i=1}^{3}\left\|w_{i}\right\|_{m}^{2}\right)^{1 / 2} .
$$

Three particular subspaces of $\mathbf{H}^{1}(\Omega)$ that satisfy specific boundary conditions are needed; they are

$$
\begin{aligned}
& \mathbf{H}_{0}^{1}(\Omega):=\left\{\mathbf{w} \in \mathbf{H}^{1}(\Omega):\left.\mathbf{w}\right|_{\partial \Omega}=0\right\} \\
& \mathbf{H}_{\tau}^{1}(\Omega):=\left\{\mathbf{w} \in \mathbf{H}^{1}(\Omega):\left.(\mathbf{w} \times \mathbf{n})\right|_{\partial \Omega}=0\right\} \\
& \mathbf{H}_{n}^{1}(\Omega):=\left\{\mathbf{w} \in \mathbf{H}^{1}(\Omega):\left.(\mathbf{w} \cdot \mathbf{n})\right|_{\partial \Omega}=0\right\}
\end{aligned}
$$


For $\mathbf{w} \in \mathbf{H}_{0}^{1}(\Omega), \mathbf{H}_{\tau}^{1}(\Omega)$, or $\mathbf{H}_{n}^{1}(\Omega)$, we will use the norm

$$
\|\mathbf{w}\|_{1}=\left(\|\mathbf{w}\|_{0}^{2}+\|\nabla \mathbf{w}\|_{0}^{2}\right)^{1 / 2}
$$

We will also make use of the product spaces

$$
\begin{aligned}
\mathscr{W}(\Omega) & :=\mathbf{H}^{1}(\Omega) \times \mathbf{H}^{1}(\Omega), \\
\mathscr{W}_{0 n}(\Omega) & :=\mathbf{H}_{0}^{1}(\Omega) \times \mathbf{H}_{n}^{1}(\Omega), \\
\mathscr{W}_{0 \tau}(\Omega) & :=\mathbf{H}_{0}^{1}(\Omega) \times \mathbf{H}_{\tau}^{1}(\Omega),
\end{aligned}
$$

all of which we will equip with the usual graph norm; i.e., if $(\mathbf{w}, \boldsymbol{\Phi}) \in \mathscr{W}(\Omega)$, $\mathscr{W}_{0 n}(\Omega)$, or $\mathscr{W}_{0 \tau}(\Omega)$, then

$$
\|(\mathbf{w}, \boldsymbol{\Phi})\|_{\mathscr{W}}:=\left(\|\mathbf{w}\|_{1}^{2}+\|\boldsymbol{\Phi}\|_{1}^{2}\right)^{1 / 2} .
$$

Next, we define

$$
\mathbf{Z}(\Omega):=\left\{\mathbf{w} \in \mathbf{H}_{0}^{1}(\Omega): \nabla \cdot \mathbf{w}=0\right\},
$$

which is a subspace of (weakly) divergence-free functions, and the subspace of $L^{2}(\Omega)$,

$$
L_{0}^{2}(\Omega):=\left\{q \in L^{2}(\Omega): \int_{\Omega} q d \mathbf{x}=0\right\},
$$

which consists of $L^{2}(\Omega)$ functions with zero mean over $\Omega$; the subspaces have norms inherited from their parent spaces. The only Sobolev space that we will need with a negative integer is $\mathbf{H}^{-1}(\Omega)$, which is defined as the dual of $\mathbf{H}_{0}^{1}(\Omega)$. The norm of a function in $\mathbf{H}^{-1}(\Omega)$ is given by

$$
\|\mathbf{f}\|_{-1}:=\sup _{\substack{\mathbf{w} \in \mathbf{H}_{0}^{1}(\Omega) \\ \mathbf{w} \neq 0}} \frac{\langle\mathbf{f}, \mathbf{w}\rangle_{\Omega}}{\|\mathbf{w}\|_{1}} .
$$

Here, $\langle\cdot, \cdot\rangle_{\Omega}$ denotes the duality pairing between the function space $\mathbf{H}_{0}^{1}(\Omega)$ and its dual. In general, $\langle\cdot, \cdot\rangle_{\Omega}$ denotes the duality pairing between a function space $V(\Omega)$ defined on the domain $\Omega$ and its dual $(V(\Omega))^{*}$, and $\langle\cdot, \cdot\rangle_{\partial \Omega}$ denotes the duality pairing between a function space $W(\partial \Omega)$ defined on the boundary $\partial \Omega$ and its dual $(W(\partial \Omega))^{*}$. The spaces $V(\Omega)$ and $W(\partial \Omega)$ will be different function spaces depending on the specific situation.

Certain trace spaces will also be needed. In particular,

$$
\begin{aligned}
H^{1 / 2}(\partial \Omega) & :=\left\{\left.w\right|_{\partial \Omega}: w \in H^{1}(\Omega)\right\}, \\
\mathbf{H}^{1 / 2}(\partial \Omega) & :=\left\{\left.\mathbf{w}\right|_{\partial \Omega}: w_{i} \in H^{1 / 2}(\partial \Omega)\right\}, \\
\mathbf{H}^{-1 / 2}(\partial \Omega) & :=\left(\mathbf{H}^{1 / 2}(\partial \Omega)\right)^{*},
\end{aligned}
$$


which are equipped with the norms

$$
\begin{aligned}
\|q\|_{1 / 2, \partial \Omega} & :=\inf _{\substack{\left.w \in H^{1}(\Omega) \\
w\right|_{\partial \Omega}=q}}\|w\|_{1}, \\
\|g\|_{1 / 2, \partial \Omega} & :=\inf _{\substack{\left.\mathbf{w} \in \mathbf{H}^{1}(\Omega) \\
\mathbf{w}\right|_{\partial \Omega}=\mathbf{g}}}\|\mathbf{w}\|_{1}, \\
\|\mathbf{k}\|_{-1 / 2, \partial \Omega} & :=\sup _{\substack{\mathbf{g} \in \mathbf{H}^{1 / 2}(\partial \Omega) \\
\mathbf{g} \neq \mathbf{0}}} \frac{\langle\mathbf{k}, \mathbf{g}\rangle_{\partial \Omega}}{\|\mathbf{g}\|_{1 / 2, \partial \Omega}},
\end{aligned}
$$

respectively. We refer the reader to [1] for further details on these spaces and their norms.

We will make use of the well-known space $L^{4}(\Omega)$, equipped with the norm $\|\cdot\|_{L^{4}}$. We also make frequent use of the following formulas of vector analysis:

$$
(\mathbf{a} \times \mathbf{b}) \times \mathbf{c} \cdot \mathbf{d}=(\mathbf{a} \times \mathbf{b}) \cdot(\mathbf{c} \times \mathbf{d})=-(\mathbf{a} \times \mathbf{b}) \cdot(\mathbf{d} \times \mathbf{c})
$$

and

$$
\int_{\Omega}(\nabla \times \boldsymbol{\Phi}) \cdot \boldsymbol{\Psi} d \mathbf{x}=-\int_{\partial \Omega}(\boldsymbol{\Phi} \times \mathbf{n}) \cdot \Psi d \mathbf{x}+\int_{\Omega} \boldsymbol{\Phi} \cdot(\nabla \times \Psi) d \mathbf{x} .
$$

2.3. Elimination of the electric field and current density. For the present, we consider the equations with the first set of boundary conditions, namely (2.8)(2.10). We require the data to have the following regularity and compatibility properties:

$$
\begin{gathered}
\mathbf{f} \in \mathbf{H}^{-1}(\Omega), \\
\mathbf{g} \in \mathbf{H}^{1 / 2}(\partial \Omega) \quad \text { with } \int_{\partial \Omega} \mathbf{g} \cdot \mathbf{n} d \mathbf{x}=0, \\
q \in H^{1 / 2}(\partial \Omega) \quad \text { with } \int_{\partial \Omega} q d \mathbf{x}=0, \\
\mathbf{k} \in \mathbf{H}^{-1 / 2}(\partial \Omega) \quad \text { with } \mathbf{k} \cdot \mathbf{n}=0,\langle\mathbf{k}, 1\rangle_{\partial \Omega}=0 \\
\text { and }\langle\mathbf{k}, \nabla \phi\rangle_{\partial \Omega}=0 \forall \phi \in H^{2}(\Omega) .
\end{gathered}
$$

In (2.14) and (2.15) the compatibility conditions on the data, i.e., $\int_{\partial \Omega} \mathbf{g} \cdot \mathbf{n} d \mathbf{x}=$ $0, \int_{\partial \Omega} q d \mathbf{x}=0$, are a result of the fact that $\nabla \cdot \mathbf{u}=0$ and $\nabla \cdot \mathbf{B}=0$ in $\Omega$; the compatibility conditions of $(2.16),\langle\mathbf{k}, 1\rangle_{\partial \Omega}=0,\langle\mathbf{k}, \nabla \phi\rangle_{\partial \Omega}=0$ for all $\phi \in H^{2}(\Omega)$, result from the equation $\nabla \times \mathbf{E}=0$ in $\Omega$ and the identities

$$
\int_{\Omega} \nabla \times \mathbf{E} d \mathbf{x}=-\left\langle\left.(\mathbf{E} \times \mathbf{n})\right|_{\partial \Omega}, 1\right\rangle_{\partial \Omega}
$$

and

$$
\int_{\Omega} \nabla \times \mathbf{E} \cdot \nabla \phi d \mathbf{x}=-\left\langle\left.(\mathbf{E} \times \mathbf{n})\right|_{\partial \Omega}, \nabla \phi\right\rangle_{\partial \Omega},
$$

and $\mathbf{k} \cdot \mathbf{n}=0$ from the fact that $(\mathbf{E} \times \mathbf{n}) \cdot \mathbf{n}=0$. 
With the aid of (2.3) and (2.5) we may replace the boundary condition (2.10) by

$$
\left.\frac{1}{R_{m}}[(\nabla \times \mathbf{B}) \times \mathbf{n}]\right|_{\partial \Omega}-\left.[(\mathbf{u} \times \mathbf{B}) \times \mathbf{n}]\right|_{\partial \Omega}=\mathbf{k} .
$$

This implies that Ohm's law, (2.3), as well as (2.5), holds up to the boundary. The substitution of (2.5) into (2.1) and (2.3) yields

$$
-\frac{1}{M^{2}} \Delta \mathbf{u}+\frac{1}{N} \mathbf{u} \cdot \nabla \mathbf{u}+\nabla p-\frac{1}{R_{m}}(\nabla \times \mathbf{B}) \times \mathbf{B}=\mathbf{f}
$$

and

$$
\nabla \times \mathbf{B}-R_{m} \mathbf{E}-R_{m}(\mathbf{u} \times \mathbf{B})=0 .
$$

Finally, $\mathbf{E}$ may be eliminated from this last equation through the use of (2.7). ing:

In summary, the system of equations that we consider is given by the follow-

along with the boundary conditions

$$
\begin{gathered}
\left.\mathbf{u}\right|_{\partial \Omega}=\mathbf{g} \quad \text { with } \int_{\partial \Omega} \mathbf{g} \cdot \mathbf{n} d \mathbf{x}=0 \\
\left.(\mathbf{B} \cdot \mathbf{n})\right|_{\partial \Omega}=q \text { with } \int_{\partial \Omega} q d \mathbf{x}=0 \\
\left.\frac{1}{R_{m}}[(\nabla \times \mathbf{B}) \times \mathbf{n}]\right|_{\partial \Omega}-\left.[(\mathbf{u} \times \mathbf{B}) \times \mathbf{n}]\right|_{\partial \Omega}=\mathbf{k} \\
\text { with } \mathbf{k} \cdot \mathbf{n}=0,\langle\mathbf{k}, 1\rangle_{\partial \Omega}=0 \\
\text { and }\langle\mathbf{k}, \nabla \phi\rangle_{\partial \Omega}=0 \forall \phi \in H^{2}(\Omega) .
\end{gathered}
$$

\section{WEAK FORMULATION}

In this section we present a new problem and show that its solutions are weak solutions of the system of differential equations and boundary conditions (2.17)-(2.23), i.e., it is a weak formulation of the original problem. Before introducing the new problem, we define the following forms. For $(\mathbf{u}, \mathbf{B}),(\mathbf{v}, \Psi)$, $(\mathbf{w}, \boldsymbol{\Phi}) \in \mathscr{W}(\Omega)$ and $\chi \in L_{0}^{2}(\Omega)$ let

$$
\begin{aligned}
a_{0}((\mathbf{u}, \mathbf{B}),(\mathbf{v}, \Psi)):=\int_{\Omega}\left\{\frac{1}{M^{2}} \nabla \mathbf{u}: \nabla \mathbf{v}+\frac{1}{R_{m}^{2}}\right. & {[(\nabla \times \mathbf{B}) \cdot(\nabla \times \Psi)} \\
& +(\nabla \cdot \mathbf{B})(\nabla \cdot \Psi)]\} d \mathbf{x},
\end{aligned}
$$




$$
\begin{aligned}
& a_{1}((\mathbf{u}, \mathbf{B}),(\mathbf{v}, \Psi),(\mathbf{w}, \boldsymbol{\Phi})) \\
& :=\int_{\Omega} \frac{1}{N} \mathbf{u} \cdot \nabla \mathbf{v} \cdot \mathbf{w} d \mathbf{x} \\
& -\int_{\Omega} \frac{1}{R_{m}}[(\nabla \times \Psi) \times \mathbf{B} \cdot \mathbf{w}-(\nabla \times \boldsymbol{\Phi}) \times \mathbf{B} \cdot \mathbf{v}] d \mathbf{x}, \\
& b((\mathbf{v}, \Psi), \chi):=-\int_{\Omega} \chi \nabla \cdot \mathbf{v} d \mathbf{x}, \\
& \quad F((\mathbf{v}, \Psi)):=\langle\mathbf{f}, \mathbf{v}\rangle_{\Omega}+\frac{1}{R_{m}}\left\langle\mathbf{k},\left.\Psi\right|_{\partial \Omega}\right\rangle_{\partial \Omega},
\end{aligned}
$$

where : denotes the scalar product from $\mathbb{R}^{3 \times 3} \times \mathbb{R}^{3 \times 3} \mapsto \mathbb{R}$, denotes the scalar product on $\mathbb{R}^{3}$, and $\times$ denotes the vector product on $\mathbb{R}^{3}$. Furthermore, let

$$
\begin{aligned}
a((\mathbf{u}, \mathbf{B}),(\mathbf{v}, \Psi),(\mathbf{w}, \boldsymbol{\Phi})):= & a_{0}((\mathbf{v}, \Psi),(\mathbf{w}, \boldsymbol{\Phi})) \\
& +a_{1}((\mathbf{u}, \mathbf{B}),(\mathbf{v}, \Psi),(\mathbf{w}, \boldsymbol{\Phi})) .
\end{aligned}
$$

Consider the following problem: find

$$
\begin{gathered}
(\mathbf{u}, \mathbf{B}) \in\left\{\mathbf{w} \in \mathbf{H}^{1}(\Omega):\left.\mathbf{w}\right|_{\partial \Omega}=\mathbf{g}\right\} \times\left\{\boldsymbol{\Phi} \in \mathbf{H}^{1}(\Omega):\left.(\boldsymbol{\Phi} \cdot \mathbf{n})\right|_{\partial \Omega}=q\right\} \\
\text { and } p \in L_{0}^{2}(\Omega)
\end{gathered}
$$

such that

$$
a((\mathbf{u}, \mathbf{B}),(\mathbf{u}, \mathbf{B}),(\mathbf{v}, \Psi))+b((\mathbf{v}, \Psi), p)=F((\mathbf{v}, \Psi)) \quad \forall(\mathbf{v}, \Psi) \in \mathscr{W}_{0 n}(\boldsymbol{\Omega})
$$

and

$$
b((\mathbf{u}, \mathbf{B}), \chi)=0 \quad \forall \chi \in L_{0}^{2}(\Omega) .
$$

We now proceed to show that a solution of the problem (3.6)-(3.8) also satisfies (2.17)-(2.20) in a weak sense and the boundary conditions (2.21)(2.23).

Proposition 3.1. If there exists a ((u, B), p) satisfying the problem (3.6)-(3.8), then it is also a weak solution of the original system given by (2.17)-(2.20) and boundary conditions (2.21)-(2.23).

The proof of this proposition requires the following result.

Lemma 3.2. If $\mathbf{B} \in \mathbf{H}^{1}(\Omega)$, then there exists a scalar $b \in H^{2}(\Omega)$ such that

$$
\nabla \cdot \nabla b=\nabla \cdot \mathbf{B} \text { and }\left.(\nabla b \cdot \mathbf{n})\right|_{\partial \Omega}=0 .
$$

Moreover, $\nabla b \in \mathbf{H}_{n}^{1}(\Omega)$.

Proof. Since $\mathbf{B} \in \mathbf{H}^{1}(\Omega)$, we have that $\nabla \cdot \mathbf{B} \in L^{2}(\Omega)$. Then the result follows easily, since it is known (see [7] or [8]) that the problem (3.9) has a solution $b \in H^{2}(\Omega)$ provided $\Omega$ is bounded and of class $C^{1,1}$, or $\Omega$ is a bounded convex polyhedron, $\Omega \subset \mathbb{R}^{3}$. Clearly, $\nabla b \in \mathbf{H}_{n}^{1}(\Omega)$. 
Proof of Proposition 3.1. Taking $\Psi=0$ in (3.7), we have

$$
\begin{aligned}
& \int_{\Omega}\left\{\frac{1}{M^{2}} \nabla \mathbf{u}: \nabla \mathbf{v}+\frac{1}{N} \mathbf{u} \cdot \nabla \mathbf{u} \cdot \mathbf{v}-p \nabla \cdot \mathbf{v}-\frac{1}{R_{m}}(\nabla \times \mathbf{B}) \times \mathbf{B} \cdot \mathbf{v}\right\} d \mathbf{x} \\
& \quad=\langle\mathbf{f}, \mathbf{v}\rangle_{\Omega} \quad \forall \mathbf{v} \in \mathbf{H}_{0}^{1}(\Omega) ;
\end{aligned}
$$

or, upon integrating by parts, we obtain

$$
\begin{aligned}
& -\frac{1}{M^{2}}\langle\Delta \mathbf{u}, \mathbf{v}\rangle_{\Omega}+\int_{\Omega}\left\{\frac{1}{N} \mathbf{u} \cdot \nabla \mathbf{u} \cdot \mathbf{v}-\frac{1}{R_{m}}(\nabla \times \mathbf{B}) \times \mathbf{B} \cdot \mathbf{v}\right\} d \mathbf{x} \\
& \quad+\langle\nabla p, \mathbf{v}\rangle_{\Omega}=\langle\mathbf{f}, \mathbf{v}\rangle_{\Omega} \quad \forall \mathbf{v} \in \mathbf{H}_{0}^{1}(\Omega),
\end{aligned}
$$

so that $(2.17)$ is satisfied in a weak sense, i.e., in $\mathbf{H}^{-1}(\Omega)$. Recall that (3.8) states that

$$
\int_{\Omega} \nabla \cdot \mathbf{u} \chi d \mathbf{x}=0 \quad \forall \chi \in L_{0}^{2}(\Omega)
$$

and since

$$
\int_{\Omega} \nabla \cdot \mathbf{u} d \mathbf{x}=\left.\int_{\partial \Omega}(\mathbf{u} \cdot \mathbf{n})\right|_{\partial \Omega} d \mathbf{x}=\int_{\partial \Omega} \mathbf{g} \cdot \mathbf{n} d \mathbf{x}=0,
$$

(2.18) certainly holds in a weak sense. Setting $\mathbf{v}=\mathbf{0}$ in (3.7), we obtain

$$
\begin{gathered}
\int_{\Omega}\left\{\frac{1}{R_{m}^{2}}[(\nabla \times \mathbf{B}) \cdot(\nabla \times \Psi)+(\nabla \cdot \mathbf{B})(\nabla \cdot \Psi)]+\frac{1}{R_{m}}(\nabla \times \Psi) \times \mathbf{B} \cdot \mathbf{u}\right\} d \mathbf{x} \\
\quad=\frac{1}{R_{m}}\left\langle\mathbf{k},\left.\Psi\right|_{\partial \Omega}\right\rangle_{\partial \Omega} \quad \forall \Psi \in \mathbf{H}_{n}^{1}(\Omega) .
\end{gathered}
$$

Now, since there exists $b \in H^{2}(\Omega)$ with $\nabla b \in \mathbf{H}_{n}^{1}(\Omega)$ and $\nabla \cdot \nabla b=\nabla \cdot \mathbf{B}$ (see (3.9)), we set $\Psi=\nabla b$ in (3.12) and obtain

$$
\int_{\Omega}(\nabla \cdot \mathbf{B})(\nabla \cdot \nabla b) d \mathbf{x}=\int_{\Omega}(\nabla \cdot \mathbf{B})(\nabla \cdot \mathbf{B}) d \mathbf{x}=R_{m}\left\langle\mathbf{k},\left.\nabla b\right|_{\partial \Omega}\right\rangle_{\partial \Omega} .
$$

By the compatibility condition on $\mathbf{k}$ given in (2.16), we then have

$$
\int_{\Omega}(\nabla \cdot \mathbf{B})(\nabla \cdot \mathbf{B}) d \mathbf{x}=0
$$

so that $\nabla \cdot \mathbf{B}=0$ almost everywhere in $\Omega$; hence,

$$
\int_{\Omega}(\nabla \cdot \mathbf{B}) \kappa d \mathbf{x}=0 \quad \forall \kappa \in L^{2}(\Omega)
$$

and (2.20) is satisfied in a weak sense. The incorporation of (3.13) into (3.12) yields

$$
\begin{gathered}
\int_{\Omega}\left\{\frac{1}{R_{m}}(\nabla \times \mathbf{B}) \cdot(\nabla \times \Psi)+(\nabla \times \Psi) \times \mathbf{B} \cdot \mathbf{u}\right\} d \mathbf{x} \\
\quad=\left\langle\mathbf{k},\left.\Psi\right|_{\partial \Omega}\right\rangle_{\partial \Omega} \quad \forall \Psi \in \mathbf{H}_{n}^{1}(\Omega)
\end{gathered}
$$


or, upon integrating by parts and restricting $\Psi$ to belong to $\mathbf{H}_{0}^{1}(\Omega)$, we obtain

$$
\frac{1}{R_{m}}\langle\nabla \times(\nabla \times \mathbf{B}), \Psi\rangle_{\Omega}-\int_{\Omega} \nabla \times(\mathbf{u} \times \mathbf{B}) \cdot \Psi d \mathbf{x}=0 \quad \forall \boldsymbol{\Psi} \in \mathbf{H}_{0}^{1}(\Omega) .
$$

Thus,

$$
\frac{1}{R_{m}} \nabla \times(\nabla \times \mathbf{B})-\nabla \times(\mathbf{u} \times \mathbf{B})=0 \quad \text { in } \mathbf{H}^{-1}(\Omega),
$$

i.e., (2.19) is satisfied in a weak sense. In fact, (3.16) also holds in $\mathbf{L}^{2}(\Omega)$, so that from (3.14) it also follows that

$$
\begin{aligned}
\int_{\Omega} & \left\{\frac{1}{R_{m}}(\nabla \times \mathbf{B}) \cdot(\nabla \times \boldsymbol{\Psi})+(\nabla \times \boldsymbol{\Psi}) \times \mathbf{B} \cdot \mathbf{u}\right\} d \mathbf{x} \\
& -\int_{\Omega}\left\{\frac{1}{R_{m}} \nabla \times(\nabla \times \mathbf{B}) \cdot \Psi-\nabla \times(\mathbf{u} \times \mathbf{B}) \cdot \boldsymbol{\Psi}\right\} d \mathbf{x} \\
= & \left\langle\mathbf{k},\left.\Psi\right|_{\partial \Omega}\right\rangle_{\partial \Omega} \quad \forall \Psi \in \mathbf{H}_{n}^{1}(\Omega)
\end{aligned}
$$

and therefore

$$
\begin{aligned}
& \left\langle\left.\frac{1}{R_{m}}[(\nabla \times \mathbf{B}) \times \mathbf{n}]\right|_{\partial \Omega}-\left.[(\mathbf{u} \times \mathbf{B}) \times \mathbf{n}]\right|_{\partial \Omega},\left.\Psi\right|_{\partial \Omega}\right\rangle_{\partial \Omega} \\
& =\left\langle\mathbf{k},\left.\Psi\right|_{\partial \Omega}\right\rangle_{\partial \Omega} \quad \forall \Psi \in \mathbf{H}_{n}^{1}(\Omega) .
\end{aligned}
$$

Thus, equation (2.23) holds in $\mathbf{H}^{-1 / 2}(\partial \Omega)$.

The boundary conditions (2.21) and (2.22) are obviously satisfied because the solution satisfies (3.6). Also, we have shown that if $((\mathbf{u}, \mathbf{B}), p)$ satisfy (3.7)-(3.8), then they satisfy the system of equations $(3.10),(3.11),(3.13)$, and (3.15). By definition, this means that they are weak solutions of $(2.17)-(2.20)$. The boundary condition (2.23) is satisfied in a weak sense because of equation (3.17) and the compatibility conditions. Thus, Proposition 3.1 is verified.

It can also be shown that weak solutions of $(2.17)-(2.20)$ with boundary conditions $(2.21)-(2.23)$ are weak solutions of $(2.1)-(2.7)$ with boundary conditions (2.8)-(2.10).

\section{EXISTENCE AND UNIQUENESS RESULTS}

4.1. Reduction to homogeneous boundary conditions. We can split the velocity into the sum of a function that satisfies the given inhomogeneous boundary conditions and a function that satisfies homogeneous boundary conditions. Let $\mathbf{u}=\hat{\mathbf{u}}+\mathbf{u}_{0}$, where $\hat{\mathbf{u}} \in \mathbf{H}_{0}^{1}(\Omega)$ and

$$
\mathbf{u}_{0} \in \mathbf{H}^{1}(\Omega), \quad \nabla \cdot \mathbf{u}_{0}=0,\left.\quad \mathbf{u}_{0}\right|_{\partial \Omega}=\mathbf{g}, \quad\left\|\mathbf{u}_{0}\right\|_{1} \leq \gamma_{1}\|\mathbf{g}\|_{1 / 2, \partial \Omega}
$$

for some positive constant $\gamma_{1}<\infty$. The existence of such a $\mathbf{u}_{0}$ can be demonstrated as follows. First, given $\mathbf{g} \in \mathbf{H}^{1 / 2}(\partial \Omega)$, there exists $\mathbf{u}_{1} \in \mathbf{H}^{1}(\Omega)$ such that

$$
\left.\mathbf{u}_{1}\right|_{\partial \Omega}=\mathbf{g} \text { and }\left\|\mathbf{u}_{1}\right\|_{1} \leq C\|\mathbf{g}\|_{1 / 2, \partial \Omega}
$$


Let $\mathbf{u}_{2}$ satisfy

$$
\nabla \cdot \mathbf{u}_{2}=-\nabla \cdot \mathbf{u}_{1} \quad \text { with }\left.\mathbf{u}_{2}\right|_{\partial \Omega}=0
$$

Since $\nabla \cdot \mathbf{u}_{1} \in L_{0}^{2}(\Omega)$, it is well known [7] that there exists such a $\mathbf{u}_{2} \in \mathbf{H}_{0}^{1}(\Omega)$ that also satisfies

$$
\left\|\mathbf{u}_{2}\right\|_{1} \leq C\left\|\nabla \cdot \mathbf{u}_{1}\right\|_{0} \leq C\left\|\mathbf{u}_{1}\right\|_{1} .
$$

Then, $\mathbf{u}_{0}=\mathbf{u}_{1}+\mathbf{u}_{2}$ is easily seen to satisfy (4.1). Likewise, for the magnetic field, let $\mathbf{B}=\widehat{\mathbf{B}}+\mathbf{B}_{0}$, where $\widehat{\mathbf{B}} \in \mathbf{H}_{n}^{1}(\Omega)$ and

$$
\begin{aligned}
& \mathbf{B}_{0} \in \mathbf{H}^{1}(\mathbf{\Omega}), \quad \nabla \cdot \mathbf{B}_{0}=0, \quad \nabla \times \mathbf{B}_{0}=0, \\
& \left.\left(\mathbf{B}_{0} \cdot \mathbf{n}\right)\right|_{\partial \Omega}=q, \quad\left\|\mathbf{B}_{0}\right\|_{1} \leq \gamma_{2}\|q\|_{1 / 2, \partial \Omega}
\end{aligned}
$$

for some positive constant $\gamma_{2}<\infty$. If $\Omega$ is bounded and of class $C^{1,1}$, we can find a $\mathbf{B}_{0}$ satisfying (4.2) by solving the problem

$$
\begin{aligned}
-\Delta b=0 & \text { on } \Omega, \\
\left.(\nabla b \cdot \mathbf{n})\right|_{\partial \Omega}=q & \text { on } \partial \Omega .
\end{aligned}
$$

In this case, $b \in H^{2}(\Omega)$ and $\|b\|_{2} \leq C\|q\|_{1 / 2, \partial \Omega}$ (see [7]). With $\mathbf{B}_{0}=\nabla b$, (4.2) is clearly satisfied. If $\Omega$ is a convex polyhedron, $q$ will have to satisfy an additional compatibility condition in order to ensure the existence of a $\mathbf{B}_{0}$ satisfying (4.2). One such condition is that $q \mathbf{n} \in \mathbf{H}^{1 / 2}(\partial \Omega)$; this condition will be satisfied if $q$ vanishes along edges and at the vertices of the polyhedron. Assume this condition holds. Note that

$$
\int_{\partial \Omega} q \mathbf{n} \cdot \mathbf{n} d \mathbf{x}=\int_{\partial \Omega} q d \mathbf{x}=0
$$

where the first equality follows from the fact that $\mathbf{n}$ is a unit vector, and the second equality holds because of the compatibility condition (2.15) on $q$. Hence, there exists $\mathbf{B}_{1} \in \mathbf{H}^{1}(\Omega)$ with $\nabla \cdot \mathbf{B}_{1}=0,\left.\mathbf{B}_{1}\right|_{\partial \Omega}=q \mathbf{n}$, and

$$
\left\|\mathbf{B}_{1}\right\|_{1} \leq C\|q\|_{1 / 2, \partial \Omega}
$$

Note that

$$
\left.\left(\mathbf{B}_{1} \cdot \mathbf{n}\right)\right|_{\partial \Omega}=\left.(q \mathbf{n} \cdot \mathbf{n})\right|_{\partial \Omega}=q,
$$

since $\mathbf{n}$ is a unit vector. Now since $\nabla \cdot\left(\nabla \times \mathbf{B}_{1}\right)=0$, it is also known (see [7]) that there exists a vector potential $\mathbf{B}_{2} \in \mathbf{H}^{1}(\Omega)$ such that

$$
\begin{gathered}
\nabla \times \mathbf{B}_{2}=\nabla \times \mathbf{B}_{1}, \quad \nabla \cdot \mathbf{B}_{2}=0,\left.\quad\left(\mathbf{B}_{2} \cdot \mathbf{n}\right)\right|_{\partial \Omega}=0, \\
\left\|\mathbf{B}_{2}\right\|_{1} \leq C\left\|\nabla \times \mathbf{B}_{1}\right\|_{0} \leq C\left\|\mathbf{B}_{1}\right\|_{1} .
\end{gathered}
$$

With $\mathbf{B}_{0}=\mathbf{B}_{1}-\mathbf{B}_{2}$, again (4.2) is clearly satisfied. 
We now rewrite (3.7) and (3.8) using the above splittings for $\mathbf{u}$ and $\mathbf{B}$. We obtain

$$
\begin{aligned}
& a\left(\left(\hat{\mathbf{u}}+\mathbf{u}_{0}, \widehat{\mathbf{B}}+\mathbf{B}_{0}\right),\left(\hat{\mathbf{u}}+\mathbf{u}_{0}, \widehat{\mathbf{B}}+\mathbf{B}_{0}\right),(\mathbf{v}, \Psi)\right)+b((\mathbf{v}, \Psi), p) \\
& \quad=F(\mathbf{v}, \Psi) \quad \forall(\mathbf{v}, \Psi) \in \mathscr{W}_{0 n}(\Omega)
\end{aligned}
$$

and

$$
b\left(\left(\hat{\mathbf{u}}+\mathbf{u}_{0}, \widehat{\mathbf{B}}+\mathbf{B}_{0}\right), \chi\right)=0 \quad \forall \chi \in L_{0}^{2}(\mathbf{\Omega})
$$

or equivalently,

$$
\begin{aligned}
& a((\hat{\mathbf{u}}, \widehat{\mathbf{B}}),(\hat{\mathbf{u}}, \widehat{\mathbf{B}}),(\mathbf{v}, \Psi))+a_{1}\left((\hat{\mathbf{u}}, \widehat{\mathbf{B}}),\left(\mathbf{u}_{0}, \mathbf{B}_{0}\right),(\mathbf{v}, \Psi)\right) \\
& \quad+a_{1}\left(\left(\mathbf{u}_{0}, \mathbf{B}_{0}\right),(\hat{\mathbf{u}}, \widehat{\mathbf{B}}),(\mathbf{v}, \Psi)\right)+\mathbf{b}((\mathbf{v}, \Psi), p) \\
& =F((\mathbf{v}, \Psi))-a\left(\left(\mathbf{u}_{0}, \mathbf{B}_{0}\right),\left(\mathbf{u}_{0}, \mathbf{B}_{0}\right),(\mathbf{v}, \Psi)\right) \quad \forall(\mathbf{v}, \Psi) \in \mathscr{W}_{0 n}(\boldsymbol{\Omega})
\end{aligned}
$$

and

$$
b((\hat{\mathbf{u}}, \widehat{\mathbf{B}}), \chi)=-b\left(\left(\mathbf{u}_{0}, \mathbf{B}_{0}\right), \chi\right)=0 \quad \forall \chi \in L_{0}^{2}(\Omega),
$$

where the last equality follows from the fact that $\nabla \cdot \mathbf{u}_{0}=0$.

4.2. Continuity and coercivity properties. We now proceed to verify certain properties of the forms which will be needed to prove the existence and uniqueness results.

Lemma 4.1. The bilinear forms $a_{0}(\cdot, \cdot)$ and $b(\cdot, \cdot)$, the trilinear form $a_{1}(\cdot, \cdot, \cdot)$, and the linear functional $F(\cdot)$ are continuous on the indicated spaces.

Proof. The results follow from the following inequalities:

$$
\begin{aligned}
\left|a_{0}((\mathbf{u}, \mathbf{B}),(\mathbf{v}, \Psi))\right| \leq & \frac{1}{M^{2}} \int_{\Omega}|\nabla \mathbf{u}: \nabla \mathbf{v}| d \mathbf{x}+\frac{1}{R_{m}^{2}} \int_{\Omega}|(\nabla \times \mathbf{B}) \cdot(\nabla \times \Psi)| d \mathbf{x} \\
& +\frac{1}{R_{m}^{2}} \int_{\Omega}|(\nabla \cdot \mathbf{B})(\nabla \cdot \Psi)| d \mathbf{x} \\
\leq & \frac{1}{M^{2}}\|\mathbf{u}\|_{1}\|\mathbf{v}\|_{1}+\frac{2}{R_{m}^{2}}\|\mathbf{B}\|_{1}\|\Psi\|_{1}+\frac{3}{R_{m}^{2}}\|\mathbf{B}\|_{1}\|\Psi\|_{1} \\
\leq & \max \left\{\frac{1}{M^{2}}, \frac{5}{R_{m}^{2}}\right\}\|(\mathbf{u}, \mathbf{B})\|_{\mathscr{W}}\|(\mathbf{v}, \Psi)\|_{\mathscr{W}},
\end{aligned}
$$


which holds for all $(\mathbf{u}, \mathbf{B}),(\mathbf{v}, \Psi) \in \mathscr{W}(\Omega)$;

$$
\begin{aligned}
& \left|a_{1}((\mathbf{u}, \mathbf{B}),(\mathbf{v}, \Psi),(\mathbf{w}, \boldsymbol{\Phi}))\right| \\
& \leq \frac{1}{N} \int_{\Omega}|\mathbf{u} \cdot \nabla \mathbf{v} \cdot \mathbf{w}| d \mathbf{x} \\
& +\frac{1}{R_{m}} \int_{\Omega}\{|(\nabla \times \mathbf{\Psi}) \cdot(\mathbf{B} \times \mathbf{w})|+|(\nabla \times \mathbf{\Phi}) \cdot(\mathbf{B} \times \mathbf{v})|\} d \mathbf{x} \\
& \leq\left[\frac{1}{N}\|\mathbf{u}\|_{\mathbf{L}^{4}}\|\mathbf{v}\|_{1}\|\mathbf{w}\|_{\mathbf{L}^{4}}+\frac{\sqrt{2}}{R_{m}}\left(\|\boldsymbol{\Psi}\|_{1}\|\mathbf{B}\|_{\mathbf{L}^{4}}\|\mathbf{w}\|_{\mathbf{L}^{4}}+\|\boldsymbol{\Phi}\|_{1}\|\mathbf{B}\|_{\mathbf{L}^{4}}\|\mathbf{v}\|_{\mathbf{L}^{4}}\right)\right] \\
& \leq \frac{\gamma_{3}}{N}\|\mathbf{u}\|_{1}\|\mathbf{v}\|_{1}\|\mathbf{w}\|_{1} \\
& +\frac{\sqrt{2} \gamma_{3}}{R_{m}}\left[\|\boldsymbol{\Psi}\|_{1}\|\mathbf{B}\|_{1}\|\mathbf{w}\|_{1}+\|\mathbf{\Phi}\|_{1}\|\mathbf{B}\|_{1}\|\mathbf{v}\|_{1}\right] \\
& \leq \frac{\gamma_{3}}{N}\|\mathbf{u}\|_{1}\|\mathbf{v}\|_{1}\|\mathbf{w}\|_{1}+\frac{\sqrt{2} \gamma_{3}}{R_{m}}\|\mathbf{B}\|_{1}\|(\mathbf{v}, \boldsymbol{\Psi})\|_{\mathscr{W}}\|(\mathbf{w}, \boldsymbol{\Phi})\|_{\mathscr{W}} \\
& \leq \max \left\{\frac{\gamma_{3}}{N}, \frac{\sqrt{2} \gamma_{3}}{R_{m}}\right\}\|(\mathbf{u}, \mathbf{B})\|_{\mathscr{W}}\left[\|\mathbf{v}\|_{1}^{2}\|\mathbf{w}\|_{1}^{2}+\|(\mathbf{v}, \Psi)\|_{\mathscr{W}}^{2}\|(\mathbf{w}, \boldsymbol{\Phi})\|_{\mathscr{W}}^{2}\right]^{1 / 2} \\
& \leq \sqrt{2} \gamma_{3} \max \left\{\frac{1}{N}, \frac{\sqrt{2}}{R_{m}}\right\}\|(\mathbf{u}, \mathbf{B})\|_{\mathscr{W}}\|(\mathbf{v}, \Psi)\|_{\mathscr{W}}\|(\mathbf{w}, \boldsymbol{\Phi})\|_{\mathscr{W}},
\end{aligned}
$$

which holds for all $(\mathbf{u}, \mathbf{B}),(\mathbf{v}, \Psi),(\mathbf{w}, \mathbf{\Phi}) \in \mathscr{W}(\Omega)$;

$$
|b((\mathbf{v}, \Psi), \chi)| \leq \int_{\Omega}|\chi \nabla \cdot \mathbf{v}| d \mathbf{x} \leq \sqrt{3}\|\chi\|_{0}\|\mathbf{v}\|_{1} \leq \sqrt{3}\|\chi\|_{0}\|(\mathbf{v}, \Psi)\|_{\mathscr{W}}
$$

which holds for all $(\mathbf{v}, \Psi) \in \mathscr{W}(\Omega)$ and $\chi \in L^{2}(\Omega)$; and

$$
\begin{aligned}
|F(\mathbf{v}, \Psi)| & \leq\left|\langle\mathbf{f}, \mathbf{v}\rangle_{\Omega}\right|+\frac{1}{R_{m}}\left|\left\langle\mathbf{k},\left.\Psi\right|_{\partial \Omega}\right\rangle_{\partial \Omega}\right| \\
& \leq\|\mathbf{f}\|_{-1}\|\mathbf{v}\|_{1}+\frac{1}{R_{m}}\|\mathbf{k}\|_{-1 / 2, \partial \Omega}\left\|\left.\Psi\right|_{\partial \Omega}\right\|_{1 / 2, \partial \Omega} \\
& \leq\left\{\|\mathbf{f}\|_{-1}+\frac{1}{R_{m}}\|\mathbf{k}\|_{-1 / 2, \partial \Omega}\right\}\|(\mathbf{v}, \Psi)\|_{\mathscr{W}},
\end{aligned}
$$

which holds for all $(\mathbf{v}, \Psi) \in \mathbf{H}_{0}^{1}(\Omega) \times \mathbf{H}^{1}(\Omega)$. In the above formulas, $\gamma_{3}=\gamma_{4}^{2}$, where $\gamma_{4}$ is the imbedding constant of $\mathbf{H}^{1}(\Omega) \hookrightarrow \mathbf{L}^{4}(\Omega)$ (here $\hookrightarrow$ denotes continuous imbedding) and $\gamma_{4}$ is a positive number. We have also made use of the following inequalities, which hold for $\mathbf{u}, \mathbf{v} \in \mathbb{R}^{3}$ :

$$
\begin{aligned}
|\mathbf{u} \cdot \mathbf{v}| & \leq\|\mathbf{u}\|_{\mathbb{R}^{3}}\|\mathbf{v}\|_{\mathbb{R}^{3}}, \\
\|\mathbf{u} \times \mathbf{v}\|_{\mathbb{R}^{3}} & \leq\|\mathbf{u}\|_{\mathbb{R}^{3}}\|\mathbf{v}\|_{\mathbb{R}^{3}}, \\
\|\nabla \times \mathbf{u}\|_{\mathbb{R}^{3}} & \leq \sqrt{2}\|\nabla \mathbf{u}\|_{\mathbb{R}^{3 \times 3}}, \\
|\nabla \cdot \mathbf{u}| & \leq \sqrt{3}\|\nabla \mathbf{u}\|_{\mathbb{R}^{3 \times 3}},
\end{aligned}
$$


and, for $S \in \mathbb{R}^{3 \times 3}$,

$$
\|S \mathbf{u}\|_{\mathbb{R}^{3}} \leq\|S\|_{\mathbb{R}^{3 \times 3}}\|\mathbf{u}\|_{\mathbb{R}^{3}}
$$

In the following two lemmas, we will show that $a_{0}(\cdot, \cdot)$ is coercive, that $b(\cdot, \cdot)$ satisfies an inf-sup condition, and that under certain restrictions $a_{1}(\cdot, \cdot, \cdot)$ is antisymmetric in its last two arguments. These conditions will eventually enable us to show the existence and uniqueness of a solution to our problem provided the data satisfies some additional constraints.

Lemma 4.2. The bilinear form $a_{0}(\cdot, \cdot)$ is coercive on $\mathscr{W}_{0 n}(\Omega)$, and the bilinear form $b(\cdot, \cdot)$ satisfies the inf-sup condition

$$
\inf _{\chi \in L_{0}^{2}(\Omega)} \sup _{(\hat{\mathbf{u}}, \widehat{\mathbf{B}}) \in \mathscr{W}_{0 n}(\Omega)} \frac{b((\hat{\mathbf{u}}, \widehat{\mathbf{B}}), \chi)}{\|(\hat{\mathbf{u}}, \widehat{\mathbf{B}})\|_{\mathscr{W}}\|\chi\|_{0}} \geq \beta .
$$

Proof. Since $(\hat{\mathbf{u}}, \widehat{\mathbf{B}}) \in \mathscr{W}_{0 n}(\Omega)$, we have that

$$
\begin{aligned}
& a_{0}((\hat{\mathbf{u}}, \widehat{\mathbf{B}}),(\hat{\mathbf{u}}, \widehat{\mathbf{B}})) \\
& \quad=\int_{\Omega}\left\{\frac{1}{M^{2}} \nabla \hat{\mathbf{u}}: \nabla \hat{\mathbf{u}}+\frac{1}{R_{m}^{2}}[(\nabla \times \widehat{\mathbf{B}}) \cdot(\nabla \times \widehat{\mathbf{B}})+(\nabla \cdot \widehat{\mathbf{B}})(\nabla \cdot \widehat{\mathbf{B}})]\right\} d \mathbf{x} \\
& \quad=\frac{1}{M^{2}}\|\nabla \hat{\mathbf{u}}\|_{0}^{2}+\frac{1}{R_{m}^{2}}\left(\|\nabla \times \widehat{\mathbf{B}}\|_{0}^{2}+\|\nabla \cdot \widehat{\mathbf{B}}\|_{0}^{2}\right) \\
& \quad \geq \frac{k_{1}}{M^{2}}\|\hat{\mathbf{u}}\|_{1}^{2}+\frac{k_{2}}{R_{m}^{2}}\|\widehat{\mathbf{B}}\|_{1}^{2} \geq \min \left(\frac{k_{1}}{M^{2}}, \frac{k_{2}}{R_{m}^{2}}\right)\|(\hat{\mathbf{u}}, \widehat{\mathbf{B}})\|_{\mathscr{W}}^{2},
\end{aligned}
$$

where $k_{1}$ is the constant in the inequality

$$
\|\nabla \mathbf{w}\|_{0}^{2} \geq k_{1}\|\mathbf{w}\|_{1}^{2} \quad \forall \mathbf{w} \in \mathbf{H}_{0}^{1}(\Omega),
$$

which follows from Poincaré's inequality; see [7] for details. Also, $k_{2}$ is the constant in the inequality

$$
\|\nabla \times \mathbf{w}\|_{0}^{2}+\|\nabla \cdot \mathbf{w}\|_{0}^{2} \geq k_{2}\|\mathbf{w}\|_{1}^{2} \quad \forall \mathbf{w} \in \mathbf{H}_{n}^{1}(\Omega) .
$$

This is a result of the imbedding $\mathbf{H}_{n}^{1}(\Omega) \hookrightarrow \mathbf{H}^{1}(\Omega)$ and of the following inequality, which holds for domains of the type being considered here (see [7]):

$$
\|\mathbf{w}\|_{0} \leq C\left(\|\nabla \times \mathbf{w}\|_{0}+\|\nabla \cdot \mathbf{w}\|_{0}\right) \quad \forall \mathbf{w} \in \mathbf{H}_{n}^{1}(\Omega) .
$$

Thus, the coercivity of the form $a_{0}(\cdot, \cdot)$ on the indicated spaces is evident. Next, there exists $\beta>0$ such that

$$
\sup _{(\hat{\mathbf{u}}, \widehat{\mathbf{B}}) \in \mathscr{W}_{0 n}} \frac{b((\hat{\mathbf{u}}, \hat{\mathbf{B}}), \chi)}{\|(\hat{\mathbf{u}}, \hat{\mathbf{B}})\|_{\mathscr{W}}} \geq \beta\|\chi\|_{0} \quad \forall \chi \in L_{0}^{2}(\Omega),
$$

since, obviously,

$$
\sup _{(\hat{\mathbf{u}}, \widehat{\mathbf{B}}) \in \mathscr{W}_{0 n}} \frac{b((\hat{\mathbf{u}}, \widehat{\mathbf{B}}), \chi)}{\|(\hat{\mathbf{u}}, \widehat{\mathbf{B}})\|_{\mathscr{W}}} \geq \sup _{(\hat{\mathbf{u}}, \widehat{\mathbf{B}}) \in \mathbf{H}_{0}^{1}(\Omega) \times\{0\}} \frac{b((\hat{\mathbf{u}}, \widehat{\mathbf{B}}), \chi)}{\|(\hat{\mathbf{u}}, \widehat{\mathbf{B}})\|_{\mathscr{W}}} \geq \beta\|\chi\|_{0} \quad \forall \chi \in L_{0}^{2}(\Omega),
$$


where the last inequality is a standard result from Navier-Stokes theory; see [7]. Dividing by $\|\chi\|_{0}$ and taking the infimum over $\chi \in L_{0}^{2}(\Omega)$ then yields (4.9).

Lemma 4.3. Let $(\mathbf{u}, \mathbf{B}),(\mathbf{v}, \Psi),(\mathbf{w}, \Phi) \in \mathscr{W}(\Omega)$ with $\nabla \cdot \mathbf{u}=0$ and at least one of $\mathbf{u}, \mathbf{v}, \mathbf{w} \in \mathbf{H}_{0}^{1}(\Omega)$. Then the trilinear form $a_{1}(\cdot, \cdot, \cdot)$ is antisymmetric with respect to its last two arguments; i.e.,

$$
a_{1}((\mathbf{u}, \mathbf{B}),(\mathbf{v}, \Psi),(\mathbf{w}, \boldsymbol{\Phi}))=-a_{1}((\mathbf{u}, \mathbf{B}),(\mathbf{w}, \boldsymbol{\Phi}),(\mathbf{v}, \Psi)) .
$$

Proof. Recall that

$$
\begin{aligned}
& a_{1}((\mathbf{u}, \mathbf{B}),(\mathbf{v}, \boldsymbol{\Psi}),(\mathbf{w}, \boldsymbol{\Phi})) \\
& \quad=\int_{\Omega}\left\{\frac{1}{N} \mathbf{u} \cdot \nabla \mathbf{v} \cdot \mathbf{w}-\frac{1}{R_{m}}[(\nabla \times \Psi) \times \mathbf{B} \cdot \mathbf{w}-(\nabla \times \mathbf{\Phi}) \times \mathbf{B} \cdot \mathbf{v}]\right\} d \mathbf{x},
\end{aligned}
$$

so it is obvious that

$$
\begin{aligned}
\int_{\Omega}\{ & \left.-\frac{1}{R_{m}}[(\nabla \times \boldsymbol{\Psi}) \times \mathbf{B} \cdot \mathbf{w}-(\nabla \times \mathbf{\Phi}) \times \mathbf{B} \cdot \mathbf{v}]\right\} d \mathbf{x} \\
& =-\int_{\Omega}\left\{-\frac{1}{R_{m}}[(\nabla \times \mathbf{\Phi}) \times \mathbf{B} \cdot \mathbf{v}-(\nabla \times \mathbf{\Psi}) \times \mathbf{B} \cdot \mathbf{w}]\right\} d \mathbf{x} .
\end{aligned}
$$

Using the divergence theorem, we conclude that

$$
\int_{\Omega}(\mathbf{u} \cdot \nabla \mathbf{v} \cdot \mathbf{w}+\mathbf{u} \cdot \nabla \mathbf{w} \cdot \mathbf{v}+(\mathbf{v} \cdot \mathbf{w}) \nabla \cdot \mathbf{u}) d \mathbf{x}=\int_{\partial \Omega}(\mathbf{v} \cdot \mathbf{w})(\mathbf{u} \cdot \mathbf{n}) d \mathbf{x},
$$

so that

$$
\int_{\Omega} \frac{1}{N} \mathbf{u} \cdot \nabla \mathbf{v} \cdot \mathbf{w} d \mathbf{x}=-\int_{\Omega} \frac{1}{N} \mathbf{u} \cdot \nabla \mathbf{w} \cdot \mathbf{v} d \mathbf{x},
$$

whenever the hypotheses are satisfied. The combination of (4.12) and (4.13) yields (4.11). Note that (4.11) implies that

$$
a_{1}((\mathbf{u}, \mathbf{B}),(\mathbf{v}, \Psi),(\mathbf{v}, \Psi))=0 .
$$

\subsection{Existence and uniqueness results. Now let}

$$
\begin{aligned}
& \hat{a}((\mathbf{u}, \mathbf{B}),(\mathbf{v}, \Psi),(\mathbf{w}, \boldsymbol{\Phi})) \\
&:= a((\mathbf{u}, \mathbf{B}),(\mathbf{v}, \Psi),(\mathbf{w}, \boldsymbol{\Phi}))+a_{1}\left((\mathbf{v}, \Psi),\left(\mathbf{u}_{0}, \mathbf{B}_{0}\right),(\mathbf{w}, \boldsymbol{\Phi})\right) \\
&+a_{1}\left(\left(\mathbf{u}_{0}, \mathbf{B}_{0}\right),(\mathbf{v}, \Psi),(\mathbf{w}, \boldsymbol{\Phi})\right) \\
&= a_{0}((\mathbf{v}, \Psi),(\mathbf{w}, \boldsymbol{\Phi}))+a_{1}((\mathbf{u}, \mathbf{B}),(\mathbf{v}, \Psi),(\mathbf{w}, \boldsymbol{\Phi})) \\
&+a_{1}\left((\mathbf{v}, \Psi),\left(\mathbf{u}_{0}, \mathbf{B}_{0}\right),(\mathbf{w}, \boldsymbol{\Phi})\right)+a_{1}\left(\left(\mathbf{u}_{0}, \mathbf{B}_{0}\right),(\mathbf{v}, \Psi),(\mathbf{w}, \boldsymbol{\Phi})\right)
\end{aligned}
$$

and

$$
\widehat{F}((\mathbf{v}, \Psi)):=F((\mathbf{v}, \Psi))-a\left(\left(\mathbf{u}_{0}, \mathbf{B}_{0}\right),\left(\mathbf{u}_{0}, \mathbf{B}_{0}\right),(\mathbf{v}, \Psi)\right),
$$

where $\mathbf{u}_{0}$ and $\mathbf{B}_{0}$ are as above, i.e., as in (4.1) and (4.2). We restate the reduced problem (4.3) and (4.4) in the following form: find $(\hat{\mathbf{u}}, \widehat{\mathbf{B}}) \in \mathscr{W}_{0 n}(\Omega)$ 
and $p \in L_{0}^{2}(\Omega)$ such that

$$
\begin{gathered}
\hat{a}((\hat{\mathbf{u}}, \widehat{\mathbf{B}}),(\hat{\mathbf{u}}, \widehat{\mathbf{B}}),(\mathbf{v}, \Psi))+b((\mathbf{v}, \Psi), p) \\
=\widehat{F}((\mathbf{v}, \Psi)) \quad \forall(\mathbf{v}, \Psi) \in \mathscr{W}_{0 n}(\boldsymbol{\Omega})
\end{gathered}
$$

and

$$
b((\hat{\mathbf{u}}, \widehat{\mathbf{B}}), \chi)=0 \quad \forall \chi \in L_{0}^{2}(\Omega) .
$$

For the existence of a solution to our problem given by (4.17) and (4.18), we must show that the linear functional $\widehat{F}(\cdot)$ is continuous on $\mathscr{W}_{0 n}(\Omega)$, that the form $\hat{a}(\cdot, \cdot, \cdot)$ is continuous on $\mathscr{W}_{0 n}(\Omega) \times \mathscr{W}_{0 n}(\Omega) \times \mathscr{W}_{0 n}(\Omega)$, and that the form $b(\cdot, \cdot)$ is continuous on $\mathscr{W}_{0 n}(\Omega) \times L_{0}^{2}(\Omega)$ and satisfies the inf-sup condition. In addition, we also will have to show that the space $Z(\Omega) \times \mathbf{H}_{n}^{1}(\Omega)$ is separable and that the form $\hat{a}(\cdot, \cdot, \cdot)$ satisfies certain coercivity and weak continuity requirements that will be made precise. Continuity of $\hat{a}(\cdot, \cdot, \cdot)$ follows trivially from the continuity of the forms $a_{0}(\cdot, \cdot)$ and $a_{1}(\cdot, \cdot, \cdot)$; the continuity of $\widehat{F}(\cdot)$ follows from the continuity of $F(\cdot), a_{0}(\cdot, \cdot)$, and $a_{1}(\cdot, \cdot, \cdot)$ (see (4.5), (4.6), and (4.8)); the continuity of the form $b(\cdot, \cdot)$ follows from (4.7); in addition, we have shown in Lemma 4.2 that $b(\cdot, \cdot)$ satisfies the necessary inf-sup condition. Clearly, the space $Z(\Omega) \times \mathbf{H}_{n}^{1}(\Omega)$ is separable, since it is a subspace of $\mathbf{H}_{0}^{1}(\Omega) \times \mathbf{H}^{1}(\Omega)$. We now must show that, under certain conditions on the data, $\hat{a}(\cdot, \cdot, \cdot)$ is coercive on $\left(\mathbf{Z}(\Omega) \times \mathbf{H}_{n}^{1}(\Omega)\right) \times\left(\mathbf{Z}(\Omega) \times \mathbf{H}_{n}^{1}(\Omega)\right) \times\left(\mathbf{Z}(\Omega) \times \mathbf{H}_{n}^{1}(\Omega)\right)$.

Lemma 4.4. There exists a constant $\alpha>0$ such that whenever

$$
\|\mathbf{g}\|_{1 / 2, \partial \Omega}<\frac{1}{\gamma_{1} \gamma_{3}} \min \left\{\frac{N k_{1}}{M^{2}}, \frac{k_{2}}{\sqrt{2} R_{m}}\right\}
$$

then

$$
\hat{a}((\hat{\mathbf{u}}, \widehat{\mathbf{B}}),(\hat{\mathbf{u}}, \widehat{\mathbf{B}}),(\hat{\mathbf{u}}, \widehat{\mathbf{B}})) \geq \alpha\|(\hat{\mathbf{u}}, \widehat{\mathbf{B}})\|_{\mathscr{W}}^{2} \quad \forall(\hat{\mathbf{u}}, \widehat{\mathbf{B}}) \in \mathbf{Z}(\boldsymbol{\Omega}) \times \mathbf{H}_{n}^{1}(\boldsymbol{\Omega}) .
$$

Proof. Through the use of (3.2), (4.14), and (4.15), one easily finds that

$$
\begin{aligned}
& \hat{a}((\hat{\mathbf{u}}, \widehat{\mathbf{B}}),(\hat{\mathbf{u}}, \widehat{\mathbf{B}}),(\hat{\mathbf{u}}, \widehat{\mathbf{B}}))-a_{0}((\hat{\mathbf{u}}, \widehat{\mathbf{B}}),(\hat{\mathbf{u}}, \widehat{\mathbf{B}})) \\
& \geq-\left|a_{1}\left((\hat{\mathbf{u}}, \widehat{\mathbf{B}}),\left(\mathbf{u}_{0}, \mathbf{B}_{0}\right),(\hat{\mathbf{u}}, \widehat{\mathbf{B}})\right)\right| \\
& =-\mid \int_{\Omega}\left\{\frac{1}{N} \hat{\mathbf{u}} \cdot \nabla \mathbf{u}_{0} \cdot \hat{\mathbf{u}}-\frac{1}{R_{m}}\left(\nabla \times \mathbf{B}_{0}\right) \times \widehat{\mathbf{B}} \cdot \hat{\mathbf{u}}\right\} d \mathbf{x} \\
& \quad+\int_{\Omega}\left\{\frac{1}{R_{m}}(\nabla \times \widehat{\mathbf{B}}) \times \widehat{\mathbf{B}} \cdot \mathbf{u}_{0}\right\} d \mathbf{x} \mid,
\end{aligned}
$$

which certainly holds for all $(\hat{\mathbf{u}}, \widehat{\mathbf{B}}) \in \mathbf{Z}(\Omega) \times \mathbf{H}_{n}^{1}(\Omega)$. From (4.2) we have that 
$\nabla \times \mathbf{B}_{0}=0$. By substituting (4.10) into the above expression, we find that

$$
\begin{aligned}
\hat{a}((\hat{\mathbf{u}}, \widehat{\mathbf{B}}),(\hat{\mathbf{u}}, \widehat{\mathbf{B}}),(\hat{\mathbf{u}}, \widehat{\mathbf{B}})) \\
\geq \frac{k_{1}}{M^{2}}\|\hat{\mathbf{u}}\|_{1}^{2}+\frac{k_{2}}{R_{m}^{2}}\|\widehat{\mathbf{B}}\|_{1}^{2} \\
\quad-\frac{1}{N}\left|\int_{\Omega} \hat{\mathbf{u}} \cdot \nabla \mathbf{u}_{0} \cdot \hat{\mathbf{u}} d \mathbf{x}\right|-\frac{1}{R_{m}}\left|\int_{\Omega}(\nabla \times \widehat{\mathbf{B}}) \times \widehat{\mathbf{B}} \cdot \mathbf{u}_{0} d \mathbf{x}\right| \\
\geq\left(\frac{k_{1}}{M^{2}}-\frac{\gamma_{3}}{N}\left\|\mathbf{u}_{0}\right\|_{1}\right)\|\hat{\mathbf{u}}\|_{1}^{2}+\left(\frac{k_{2}}{R_{m}^{2}}-\frac{\sqrt{2} \gamma_{3}}{R_{m}}\left\|\mathbf{u}_{0}\right\|_{1}\right)\|\widehat{\mathbf{B}}\|_{1}^{2} \\
\geq\left(\frac{k_{1}}{M^{2}}-\frac{\gamma_{1} \gamma_{3}}{N}\|\mathbf{g}\|_{1 / 2, \partial \Omega}\right)\|\hat{\mathbf{u}}\|_{1}^{2}+\left(\frac{k_{2}}{R_{m}^{2}}-\frac{\sqrt{2} \gamma_{1} \gamma_{3}}{R_{m}}\|\mathbf{g}\|_{1 / 2, \partial \Omega}\right)\|\widehat{\mathbf{B}}\|_{1}^{2},
\end{aligned}
$$

where we have used the bounds from (4.6) and the bound for $\left\|\mathbf{u}_{0}\right\|_{1}$ from (4.1). Clearly, (4.19) then implies that (4.20) is valid with

$$
\alpha=\min \left\{\frac{k_{1}}{M^{2}}-\frac{\gamma_{1} \gamma_{3}}{N}\|\mathbf{g}\|_{1 / 2, \partial \Omega}, \frac{k_{2}}{R_{m}^{2}}-\frac{\sqrt{2} \gamma_{1} \gamma_{3}}{R_{m}}\|\mathbf{g}\|_{1 / 2, \partial \Omega}\right\} .
$$

It is interesting to note that the restriction (4.19) on the data $\mathbf{g}$ for the velocity can be removed for two-dimensional problems. Indeed, the result of Lemma 4.4 is valid in $\mathbb{R}^{2}$, for any data $\mathbf{g}$. Since here we are interested in three-dimensional problems, we do not consider the two-dimensional case any further.

The last ingredient needed to prove the existence of a solution to (4.17)(4.18) is the following lemma.

\section{Lemma 4.5. The map}

$$
(\hat{\mathbf{u}}, \widehat{\mathbf{B}}) \in \mathbf{Z}(\Omega) \times \mathbf{H}_{n}^{1}(\Omega) \mapsto \hat{a}((\hat{\mathbf{u}}, \widehat{\mathbf{B}}),(\hat{\mathbf{u}}, \widehat{\mathbf{B}}),(\mathbf{v}, \Psi))
$$

is weakly sequentially continuous on $\mathbf{Z}(\Omega) \times \mathbf{H}_{n}^{1}(\Omega)$; i.e.,

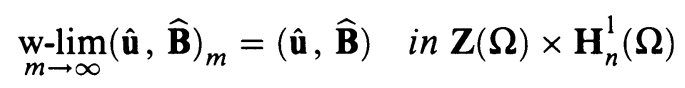

implies

$$
\begin{aligned}
\lim _{m \rightarrow \infty} & \hat{a}\left((\hat{\mathbf{u}}, \widehat{\mathbf{B}})_{m},(\hat{\mathbf{u}}, \widehat{\mathbf{B}})_{m},(\mathbf{v}, \Psi)\right) \\
\quad= & \hat{a}((\hat{\mathbf{u}}, \widehat{\mathbf{B}}),(\hat{\mathbf{u}}, \widehat{\mathbf{B}}),(\mathbf{v}, \Psi)) \quad \forall(\mathbf{v}, \Psi) \in \mathbf{Z}(\Omega) \times \mathbf{H}_{n}^{1}(\Omega) .
\end{aligned}
$$

(Here w-lim denotes the weak limit; see [7].)

Proof. If $(\hat{\mathbf{u}}, \widehat{\mathbf{B}})_{m} \rightarrow(\hat{\mathbf{u}}, \widehat{\mathbf{B}})$ in $\mathbf{Z}(\boldsymbol{\Omega}) \times \mathbf{H}_{n}^{1}(\boldsymbol{\Omega})$ (here $\rightarrow$ denotes weak convergence), then it follows that $\hat{\mathbf{u}}_{m}-\hat{\mathbf{u}}$ in $\mathbf{Z}(\Omega)$, that $\widehat{\mathbf{B}}_{m} \rightarrow \widehat{\mathbf{B}}$ in $\mathbf{H}_{n}^{1}(\Omega)$, and that $\left\{\hat{\mathbf{u}}_{m}\right\}$ and $\left\{\widehat{\mathbf{B}}_{m}\right\}$ are bounded in $\mathbf{Z}(\Omega)$ and $\mathbf{H}_{n}^{1}(\Omega)$, respectively. Since $\mathbf{H}^{1}(\Omega) \hookrightarrow \hookrightarrow \mathbf{L}^{4}(\Omega)$ (here $\hookrightarrow \hookrightarrow$ denotes compact imbedding), we have that $\hat{\mathbf{u}}_{m} \rightarrow \hat{\mathbf{u}}$ and $\widehat{\mathbf{B}}_{m} \rightarrow \widehat{\mathbf{B}}$ in $\mathbf{L}^{4}(\Omega)$ (here $\rightarrow$ denotes strong convergence), and also that $D^{\gamma} \hat{\mathbf{u}}_{m} \rightarrow D^{\gamma} \hat{\mathbf{u}}$ and $D^{\gamma} \widehat{\mathbf{B}}_{m} \rightarrow D^{\gamma} \widehat{\mathbf{B}}$ in $\mathbf{L}^{2}(\Omega)$ for all $|\gamma| \leq 1$. 
Therefore,

$$
\begin{aligned}
& \left|\hat{a}\left((\hat{\mathbf{u}}, \widehat{\mathbf{B}})_{m},(\hat{\mathbf{u}}, \widehat{\mathbf{B}})_{m},(\mathbf{v}, \Psi)\right)-\hat{a}((\hat{\mathbf{u}}, \widehat{\mathbf{B}}),(\hat{\mathbf{u}}, \widehat{\mathbf{B}}),(\mathbf{v}, \Psi))\right| \\
& =\mid a\left((\hat{\mathbf{u}}, \widehat{\mathbf{B}})_{m},(\hat{\mathbf{u}}, \widehat{\mathbf{B}})_{m},(\mathbf{v}, \Psi)\right)-a((\hat{\mathbf{u}}, \widehat{\mathbf{B}}),(\hat{\mathbf{u}}, \widehat{\mathbf{B}}),(\mathbf{v}, \Psi)) \\
& +a_{1}\left((\hat{\mathbf{u}}, \widehat{\mathbf{B}})_{m},\left(\mathbf{u}_{0}, \mathbf{B}_{0}\right),(\mathbf{v}, \Psi)\right)-a_{1}\left((\hat{\mathbf{u}}, \widehat{\mathbf{B}}),\left(\mathbf{u}_{0}, \mathbf{B}_{0}\right),(\mathbf{v}, \Psi)\right) \\
& +a_{1}\left(\left(\mathbf{u}_{0}, \mathbf{B}_{0}\right),(\hat{\mathbf{u}}, \widehat{\mathbf{B}})_{m},(\mathbf{v}, \Psi)\right)-a_{1}\left(\left(\mathbf{u}_{0}, \mathbf{B}_{0}\right),(\hat{\mathbf{u}}, \widehat{\mathbf{B}}),(\mathbf{v}, \Psi)\right) \mid \\
& =\mid a_{0}\left((\hat{\mathbf{u}}, \widehat{\mathbf{B}})_{m},(\mathbf{v}, \Psi)\right)-a_{0}((\hat{\mathbf{u}}, \widehat{\mathbf{B}}),(\mathbf{v}, \Psi)) \\
& +a_{1}\left((\hat{\mathbf{u}}, \widehat{\mathbf{B}})_{m},(\hat{\mathbf{u}}, \widehat{\mathbf{B}})_{m},(\mathbf{v}, \Psi)\right)-a_{1}((\hat{\mathbf{u}}, \widehat{\mathbf{B}}),(\hat{\mathbf{u}}, \widehat{\mathbf{B}}),(\mathbf{v}, \Psi)) \\
& +a_{1}\left((\hat{\mathbf{u}}, \widehat{\mathbf{B}})_{m},\left(\mathbf{u}_{0}, \mathbf{B}_{0}\right),(\mathbf{v}, \Psi)\right)-a_{1}\left((\hat{\mathbf{u}}, \widehat{\mathbf{B}}),\left(\mathbf{u}_{0}, \mathbf{B}_{0}\right),(\mathbf{v}, \Psi)\right) \\
& +a_{1}\left(\left(\mathbf{u}_{0}, \mathbf{B}_{0}\right),(\hat{\mathbf{u}}, \widehat{\mathbf{B}})_{m},(\mathbf{v}, \Psi)\right)-a_{1}\left(\left(\mathbf{u}_{0}, \mathbf{B}_{0}\right),(\hat{\mathbf{u}}, \widehat{\mathbf{B}}),(\mathbf{v}, \Psi)\right) \\
& =\mid a_{0}\left((\hat{\mathbf{u}}, \widehat{\mathbf{B}})_{m}-(\hat{\mathbf{u}}, \widehat{\mathbf{B}}),(\mathbf{v}, \Psi)\right)+a_{1}\left((\hat{\mathbf{u}}, \widehat{\mathbf{B}})_{m}-(\hat{\mathbf{u}}, \hat{\mathbf{B}}),(\hat{\mathbf{u}}, \hat{\mathbf{B}})_{m},(\mathbf{v}, \Psi)\right) \\
& +a_{1}\left((\hat{\mathbf{u}}, \widehat{\mathbf{B}}),(\hat{\mathbf{u}}, \widehat{\mathbf{B}})_{m}-(\hat{\mathbf{u}}, \widehat{\mathbf{B}}),(\mathbf{v}, \Psi)\right) \\
& +a_{1}\left((\hat{\mathbf{u}}, \widehat{\mathbf{B}})_{m}-(\hat{\mathbf{u}}, \widehat{\mathbf{B}}),\left(\mathbf{u}_{0}, \mathbf{B}_{0}\right),(\mathbf{v}, \Psi)\right) \\
& +a_{1}\left(\left(\mathbf{u}_{0}, \mathbf{B}_{0}\right),(\hat{\mathbf{u}}, \widehat{\mathbf{B}})_{m}-(\hat{\mathbf{u}}, \widehat{\mathbf{B}}),(\mathbf{v}, \Psi)\right) \\
& \leq \frac{1}{M^{2}}\left|\int_{\Omega}\left(\nabla \hat{\mathbf{u}}_{m}-\nabla \hat{\mathbf{u}}\right): \nabla \mathbf{v} d \mathbf{x}\right|+\frac{1}{R_{m}^{2}}\left|\int_{\Omega}\left(\nabla \times \widehat{\mathbf{B}}_{m}-\nabla \times \widehat{\mathbf{B}}\right) \cdot(\nabla \times \Psi) d \mathbf{x}\right| \\
& +\frac{1}{R_{m}^{2}}\left|\int_{\Omega}\left(\nabla \cdot \widehat{\mathbf{B}}_{m}-\nabla \cdot \widehat{\mathbf{B}}\right)(\nabla \cdot \Psi) d \mathbf{x}\right|+\frac{1}{N}\left\|\hat{\mathbf{u}}_{m}-\hat{\mathbf{u}}\right\|_{\mathbf{L}^{4}}\left\|\hat{\mathbf{u}}_{m}\right\|_{1}\|\mathbf{v}\|_{\mathbf{L}^{4}} \\
& +\frac{\sqrt{2}}{R_{m}}\left[\left\|\widehat{\mathbf{B}}_{m}\right\|_{1}\left\|\widehat{\mathbf{B}_{m}}-\widehat{\mathbf{B}}\right\|_{\mathbf{L}^{4}}\|\mathbf{v}\|_{\mathbf{L}^{4}}+\|\Psi\|_{1}\left\|\widehat{\mathbf{B}}_{m}-\widehat{\mathbf{B}}\right\|_{\mathbf{L}^{4}}\left\|\hat{\mathbf{u}}_{m}\right\|_{\mathbf{L}^{4}}\right] \\
& +\frac{1}{N}\left|\int_{\Omega} \hat{\mathbf{u}} \cdot\left(\nabla \hat{\mathbf{u}}_{m}-\nabla \hat{\mathbf{u}}\right) \cdot \mathbf{v} d \mathbf{x}\right|+\frac{1}{R_{m}}\left|\int_{\Omega}\left(\nabla \times \widehat{\mathbf{B}}_{m}-\nabla \times \widehat{\mathbf{B}}\right) \cdot(\widehat{\mathbf{B}} \times \mathbf{v}) d \mathbf{x}\right| \\
& +\frac{\sqrt{2}}{R_{m}}\|\Psi\|_{1}\|\widehat{\mathbf{B}}\|_{\mathbf{L}^{4}}\left\|\hat{\mathbf{u}}_{m}-\hat{\mathbf{u}}\right\|_{\mathbf{L}^{4}}+\frac{1}{N}\left\|\hat{\mathbf{u}}_{m}-\hat{\mathbf{u}}\right\|_{\mathbf{L}^{4}}\left\|\mathbf{u}_{0}\right\|_{1}\|\mathbf{v}\|_{\mathbf{L}^{4}} \\
& +\frac{\sqrt{2}}{R_{m}}\left[\left\|\mathbf{B}_{0}\right\|_{1}\left\|\widehat{\mathbf{B}_{m}}-\widehat{\mathbf{B}}\right\|_{\mathbf{L}^{4}}\|\mathbf{v}\|_{\mathbf{L}^{4}}+\|\Psi\|_{1}\left\|\widehat{\mathbf{B}}_{m}-\widehat{\mathbf{B}}\right\|_{\mathbf{L}^{4}}\left\|\mathbf{u}_{0}\right\|_{\mathbf{L}^{4}}\right] \\
& +\frac{1}{N}\left|\int_{\Omega} \mathbf{u}_{0} \cdot\left(\nabla \hat{\mathbf{u}}_{m}-\nabla \hat{\mathbf{u}}\right) \cdot \mathbf{v} d \mathbf{x}\right| \\
& +\frac{1}{R_{m}}\left|\int_{\Omega}\left(\nabla \times \widehat{\mathbf{B}}_{m}-\nabla \times \widehat{\mathbf{B}}\right) \cdot\left(\mathbf{B}_{0} \times \mathbf{v}\right) d \mathbf{x}\right| \\
& +\frac{\sqrt{2}}{R_{m}}\|\Psi\|_{1}\left\|\mathbf{B}_{0}\right\|_{\mathbf{L}^{4}}\left\|\hat{\mathbf{u}}_{m}-\hat{\mathbf{u}}\right\|_{\mathbf{L}^{4}} \\
& \rightarrow 0 \quad \text { as } m \rightarrow \infty \text {. }
\end{aligned}
$$

Lemmas 4.1-4.4 lead to the following existence theorem.

Theorem 4.6. Given $\mathbf{f}, q$, and $\mathbf{k}$ satisfying (2.13), (2.15), and (2.16), respectively, and $\mathbf{g}$ satisfying (2.14) and (4.19), there exists at least one $((\mathbf{u}, \mathbf{B}), p)$ 
satisfying (3.6)-(3.8). Moreover,

$$
\begin{aligned}
\|(\mathbf{u}, \mathbf{B})\|_{\mathscr{W}} \leq & \frac{1}{\alpha}\left[\|\mathbf{f}\|_{-1}+\frac{1}{R_{m}}\|\mathbf{k}\|_{-1 / 2, \partial \Omega}+\frac{\gamma_{1} \gamma_{2} \gamma_{3}}{\sqrt{2} R_{m}}\|q\|_{1 / 2, \partial \Omega}^{2}\right. \\
& \left.\quad+\gamma_{1} \gamma_{3}\left(\frac{\gamma_{1}}{N}+\frac{\gamma_{2}}{\sqrt{2} R_{m}}\right)\|\mathbf{g}\|_{1 / 2, \partial \Omega}^{2}\right] \\
& +\gamma_{2}\left(1+\frac{5}{\alpha R_{m}^{2}}\right)\|q\|_{1 / 2, \partial \Omega}+\gamma_{1}\left(1+\frac{1}{\alpha M^{2}}\right)\|\mathbf{g}\|_{1 / 2, \partial \Omega}
\end{aligned}
$$

and

$$
\begin{aligned}
\|p\|_{0} \leq \frac{1}{\beta}[\max & \left\{\frac{1}{M^{2}}, \frac{5}{R_{m}^{2}}\right\}\|(\mathbf{u}, \mathbf{B})\|_{\mathscr{W}} \\
+\sqrt{2} \gamma_{3} \max & \left\{\frac{1}{N}, \frac{\sqrt{2}}{R_{m}}\right\}\|(\mathbf{u}, \mathbf{B})\|_{\mathscr{W}}^{2} \\
+ & \left.\|\mathbf{f}\|_{-1}+\frac{1}{R_{m}}\|\mathbf{k}\|_{-1 / 2, \partial \Omega}\right] .
\end{aligned}
$$

Proof. The existence of a $((\hat{\mathbf{u}}, \widehat{\mathbf{B}}), p) \in \mathbf{Z}(\Omega) \times \mathbf{H}_{n}^{1}(\Omega) \times L_{0}^{2}(\Omega)$ satisfying (4.17) and (4.18) follows easily from Lemmas 4.1-4.5; see [7]. Moreover, we also have [7]

Then, by (4.16), we have that

$$
\|(\hat{\mathbf{u}}, \widehat{\mathbf{B}})\|_{\mathscr{W}} \leq \frac{1}{\alpha}\left[\sup _{(\mathbf{v}, \Psi) \in \mathscr{W}_{0 n}(\Omega)} \frac{\widehat{F}((\mathbf{v}, \Psi))}{\|(\mathbf{v}, \Psi)\|_{\mathscr{W}}}\right] .
$$

$$
\begin{aligned}
\|(\hat{\mathbf{u}}, \widehat{\mathbf{B}})\|_{\mathscr{W}} \leq \frac{1}{\alpha}[ & \sup _{(\mathbf{v}, \Psi) \in \mathscr{W}_{0 n}(\Omega)} \frac{|F((\mathbf{v}, \Psi))|}{\|(\mathbf{v}, \Psi)\|_{\mathscr{W}}} \\
& \left.+\sup _{(\mathbf{v}, \Psi) \in \mathscr{W}_{0 n}(\Omega)} \frac{\left|a\left(\left(\mathbf{u}_{0}, \mathbf{B}_{0}\right),\left(\mathbf{u}_{0}, \mathbf{B}_{0}\right),(\mathbf{v}, \Psi)\right)\right|}{\|(\mathbf{v}, \Psi)\|_{\mathscr{W}}}\right] .
\end{aligned}
$$

Through the use of $(3.5)$ to write $a(\cdot, \cdot, \cdot)$ in terms of $a_{0}(\cdot, \cdot)$ and $a_{1}(\cdot, \cdot, \cdot)$, (4.2) and the continuity conditions (4.5), (4.6), and (4.8), one easily finds from (4.24) that

$$
\begin{aligned}
\|(\hat{\mathbf{u}}, \widehat{\mathbf{B}})\|_{\mathscr{W}} \leq \frac{1}{\alpha}\left[\|\mathbf{f}\|_{-1}+\frac{1}{R_{m}}\|\mathbf{k}\|_{-1 / 2, \partial \Omega}\right. & +\frac{1}{M^{2}}\left\|\mathbf{u}_{0}\right\|_{1}+\frac{5}{R_{m}^{2}}\left\|\mathbf{B}_{0}\right\|_{1} \\
& \left.+\frac{\gamma_{3}}{N}\left\|\mathbf{u}_{0}\right\|_{1}^{2}+\frac{\sqrt{2} \gamma_{3}}{R_{m}}\left\|\mathbf{u}_{0}\right\|_{1}\left\|\mathbf{B}_{0}\right\|_{1}\right] .
\end{aligned}
$$

But $\mathbf{u}=\hat{\mathbf{u}}+\mathbf{u}_{0}$ and $\mathbf{B}=\widehat{\mathbf{B}}+\mathbf{B}_{0}$, so that the triangle inequality and (4.1), (4.2), i.e., $\left\|\mathbf{u}_{0}\right\|_{1} \leq \gamma_{1}\|\mathbf{g}\|_{1 / 2, \partial \Omega}$ and $\left\|\mathbf{B}_{0}\right\|_{1} \leq \gamma_{2}\|q\|_{1 / 2, \partial \Omega}$, yields (4.22). Finally, from (3.5) and (3.7) we have

$$
\begin{aligned}
b((\mathbf{v}, \Psi), p)= & -a_{0}((\mathbf{u}, \mathbf{B}),(\mathbf{v}, \Psi))-a_{1}((\mathbf{u}, \mathbf{B}),(\mathbf{u}, \mathbf{B}),(\mathbf{v}, \Psi)) \\
& +F((\mathbf{v}, \Psi)) \quad \forall(\mathbf{v}, \Psi) \in \mathscr{W}_{0 n}(\boldsymbol{\Omega}) .
\end{aligned}
$$


Thus, given $(\mathbf{u}, \mathbf{B}) \in \mathscr{W}(\Omega)$, it follows from (4.9) that there exists a unique $p \in L_{0}^{2}(\Omega)$ satisfying (3.7). Moreover, using (4.5), (4.6), (4.8), and (4.9), we easily arrive at (4.23).

It should be noted that there is no restriction on the size of $q$ or $\mathbf{k}$, the data for $\left.(\mathbf{B} \cdot \mathbf{n})\right|_{\partial \Omega}$ and $\left.(\mathbf{E} \times \mathbf{n})\right|_{\partial \Omega}$ on the boundary, respectively. Thus, if $\mathbf{g}=0$, i.e., the boundary $\partial \Omega$ is a solid wall and a no-slip condition is satisfied at the wall, we have existence regardless of the size of $q$ or $\mathbf{k}$.

For the uniqueness of the solution to the problem (3.6)-(3.8), we have the following theorem.

Theorem 4.7. There exists at most one solution $((\mathbf{u}, \mathbf{B}), p) \in \mathscr{W}(\Omega) \times L_{0}^{2}(\Omega)$ to the problem (3.6)-(3.8) such that

$$
\|(\mathbf{u}, \mathbf{B})\|_{\mathscr{W}}<\frac{1}{\sqrt{2} \gamma_{3}} \min \left\{\frac{k_{1}}{M^{2}}, \frac{k_{2}}{R_{m}^{2}}\right\} / \max \left\{\frac{1}{N}, \frac{\sqrt{2}}{R_{m}}\right\} .
$$

Moreover, if the data $\mathbf{f}, \mathbf{g}, q$, and $\mathbf{k}$ satisfy (4.19), the hypotheses of Theorem 4.6, and

$$
\begin{aligned}
\frac{1}{\alpha}\left[\|\mathbf{f}\|_{-1}\right. & +\frac{1}{R_{m}}\|\mathbf{k}\|_{-1 / 2, \partial \Omega}+\frac{\gamma_{1} \gamma_{2} \gamma_{3}}{\sqrt{2} R_{m}}\|q\|_{1 / 2, \partial \Omega}^{2} \\
& \left.+\gamma_{1} \gamma_{3}\left(\frac{\gamma_{1}}{N}+\frac{\gamma_{2}}{\sqrt{2} R_{m}}\right)\|\mathbf{g}\|_{1 / 2, \partial \Omega}^{2}\right] \\
& +\gamma_{2}\left(1+\frac{5}{\alpha R_{m}^{2}}\right)\|q\|_{1 / 2, \partial \Omega}+\gamma_{1}\left(1+\frac{1}{\alpha M^{2}}\right)\|\mathbf{g}\|_{1 / 2, \partial \Omega} \\
< & \frac{1}{\sqrt{2} \gamma_{3}} \min \left\{\frac{k_{1}}{M^{2}}, \frac{k_{2}}{R_{m}^{2}}\right\} / \max \left\{\frac{1}{N}, \frac{\sqrt{2}}{R_{m}}\right\},
\end{aligned}
$$

then the problem (3.6)-(3.8) has exactly one solution $((\mathbf{u}, \mathbf{B}), p) \in \mathscr{W}(\Omega) \times$ $L_{0}^{2}(\Omega)$.

Proof. Suppose $((\mathbf{u}, \mathbf{B}), p),((\mathbf{w}, \boldsymbol{\Phi}), s) \in \mathscr{W}(\boldsymbol{\Omega}) \times L_{0}^{2}(\Omega)$ are two, nonidentical solutions of (3.6)-(3.8). Then $(\mathbf{u}-\mathbf{w}, \mathbf{B}-\boldsymbol{\Phi}) \in \mathscr{W}_{0 n}(\Omega)$. In fact, from (3.8),

$$
b((\mathbf{u}-\mathbf{w}, \mathbf{B}-\boldsymbol{\Phi}), \chi)=b((\mathbf{u}, \mathbf{B}), \chi)-b((\mathbf{w}, \boldsymbol{\Phi}), \chi)=0 \quad \forall \chi \in L_{0}^{2}(\boldsymbol{\Omega}),
$$

so that $(\mathbf{u}-\mathbf{w}, \mathbf{B}-\boldsymbol{\Phi}) \in \mathbf{Z}(\Omega) \times \mathbf{H}_{n}^{1}(\Omega)$. Since both candidate solutions satisfy (3.7), we have

$$
a((\mathbf{u}, \mathbf{B}),(\mathbf{u}, \mathbf{B}),(\mathbf{v}, \Psi))+b((\mathbf{v}, \Psi), p)=F((\mathbf{v}, \Psi)) \quad \forall(\mathbf{v}, \Psi) \in \mathscr{W}_{0 n}(\boldsymbol{\Omega})
$$

and

$$
a((\mathbf{w}, \boldsymbol{\Phi}),(\mathbf{w}, \boldsymbol{\Phi}),(\mathbf{v}, \boldsymbol{\Psi}))+b((\mathbf{v}, \Psi), s)=F((\mathbf{v}, \Psi)) \quad \forall(\mathbf{v}, \Psi) \in \mathscr{W}_{0 n}(\boldsymbol{\Omega}) .
$$

Upon taking the difference of the last two equations and then setting $(\mathbf{v}, \Psi)=$ $(\mathbf{u}-\mathbf{w}, \mathbf{B}-\boldsymbol{\Phi})$, we have, using (3.5), (4.11), and the definition of $\mathbf{Z}(\Omega)$, that

$$
\begin{aligned}
& a_{0}((\mathbf{u}-\mathbf{w}, \mathbf{B}-\boldsymbol{\Phi}),(\mathbf{u}-\mathbf{w}, \mathbf{B}-\boldsymbol{\Phi})) \\
& \quad+a_{1}((\mathbf{u}-\mathbf{w}, \mathbf{B}-\boldsymbol{\Phi}),(\mathbf{u}, \mathbf{B}),(\mathbf{u}-\mathbf{w}, \mathbf{B}-\boldsymbol{\Phi}))=0 .
\end{aligned}
$$


Then (4.6) and (4.10) yield

$$
\begin{aligned}
\frac{k_{1}}{M^{2}}\|\mathbf{u}-\mathbf{w}\|_{1}^{2}+\frac{k_{2}}{R_{m}^{2}}\|\mathbf{B}-\boldsymbol{\Phi}\|_{1}^{2}-\frac{\gamma_{3}}{N}\|\mathbf{u}\|_{1}\|\mathbf{u}-\mathbf{w}\|_{1}^{2} \\
-\frac{\sqrt{2} \gamma_{3}}{R_{m}}\left[\|\mathbf{B}\|_{1}\|\mathbf{u}-\mathbf{w}\|_{1}\|\mathbf{B}-\boldsymbol{\Phi}\|_{1}+\|\mathbf{u}\|_{1}\|\mathbf{B}-\boldsymbol{\Phi}\|_{1}^{2}\right] \leq 0
\end{aligned}
$$

or

$$
\begin{aligned}
& \left(\frac{k_{1}}{M^{2}}-\frac{\gamma_{3}}{N}\|\mathbf{u}\|_{1}-\frac{\gamma_{3}}{\sqrt{2} R_{m}}\|\mathbf{B}\|_{1}\right)\|\mathbf{u}-\mathbf{w}\|_{1}^{2} \\
& \quad+\left(\frac{k_{2}}{R_{m}^{2}}-\frac{\sqrt{2} \gamma_{3}}{R_{m}}\|\mathbf{u}\|_{1}-\frac{\gamma_{3}}{\sqrt{2} R_{m}}\|\mathbf{B}\|_{1}\right)\|\mathbf{B}-\mathbf{\Phi}\|_{1}^{2} \leq 0 .
\end{aligned}
$$

From (4.25) we have

$$
\frac{\gamma_{3}}{N}\|\mathbf{u}\|_{1}+\frac{\gamma_{3}}{\sqrt{2} R_{m}}\|\mathbf{B}\|_{1} \leq \sqrt{2} \gamma_{3} \max \left\{\frac{1}{N}, \frac{\sqrt{2}}{R_{m}}\right\}\|(\mathbf{u}, \mathbf{B})\|_{\mathscr{W}}<\frac{k_{1}}{M^{2}}
$$

and

$$
\frac{\sqrt{2} \gamma_{3}}{R_{m}}\|\mathbf{u}\|_{1}+\frac{\gamma_{3}}{\sqrt{2} R_{m}}\|\mathbf{B}\|_{1} \leq \sqrt{2} \gamma_{3} \max \left\{\frac{1}{N}, \frac{\sqrt{2}}{R_{m}}\right\}\|(\mathbf{u}, \mathbf{B})\|_{\mathscr{W}}<\frac{k_{2}}{R_{m}^{2}} .
$$

The combination of (4.27) $-(4.29)$ easily yields $\|\mathbf{u}-\mathbf{w}\|_{1}=0$ and $\|\mathbf{B}-\boldsymbol{\Phi}\|_{1}=0$, contradicting the assumptions that $\mathbf{u} \neq \mathbf{w}$ and $\mathbf{B} \neq \boldsymbol{\Phi}$. Once $(\mathbf{u}, \mathbf{B})$ is uniquely determined, the uniqueness of $p \in L_{0}^{2}(\Omega)$ follows; see Theorem 4.6. Now, if (4.19) is satisfied, $\alpha$ given by (4.21) is positive and by Theorem 4.6 , there exists a $((\mathbf{u}, \mathbf{B}), p)$ satisfying (4.22). Then the latter and (4.26) imply that (4.25) is true so that this solution is unique.

We remark that in order for condition (4.26) to hold, all the data $\mathbf{f}, \mathbf{g}, q$, and $\mathbf{k}$ must be sufficiently small.

\section{Alternative BOUNDARY CONDITIONS}

We now consider the boundary conditions (2.8), (2.11), and (2.12). The governing system is now given by $(2.17)-(2.21)$ and

$$
\left.(\mathbf{B} \times \mathbf{n})\right|_{\partial \Omega}=\mathbf{q} \quad \text { with } \mathbf{q} \cdot \mathbf{n}=0,
$$

where again we require the data to have the following regularity: $\mathbf{f}, \mathbf{g}$ should satisfy (2.13) and (2.14), respectively, and $\mathbf{q}$ should satisfy $\mathbf{q} \in \mathbf{H}^{1 / 2}(\partial \Omega)$. The compatibility condition in $(5.1)$, i.e., $\mathbf{q} \cdot \mathbf{n}=0$, results from the fact that (obviously) $\left.(\mathbf{B} \times \mathbf{n})\right|_{\partial \Omega}$, and therefore $\mathbf{q}$, lies in the tangent plane to the boundary $\partial \Omega$.

Let $(\mathbf{u}, \mathbf{B}),(\mathbf{v}, \Psi),(\mathbf{w}, \boldsymbol{\Phi}) \in \mathscr{W}_{0 \tau}(\Omega)$ and $\chi \in L_{0}^{2}(\Omega)$. Then we define the forms $a_{0}((\mathbf{u}, \mathbf{B}),(\mathbf{v}, \Psi)), a_{1}((\mathbf{u}, \mathbf{B}),(\mathbf{v}, \Psi),(\mathbf{w}, \boldsymbol{\Phi}))$, and $b((\mathbf{v}, \Psi), \chi)$ as before (see $(3.1)-(3.3))$, and we now define $F(\cdot)$ by

$$
F((\mathbf{v}, \Psi)):=\langle\mathbf{f}, \mathbf{v}\rangle_{\Omega} \text {. }
$$


Consider the following problem: find

$$
\begin{gathered}
(\mathbf{u}, \mathbf{B}) \in\left\{\mathbf{w} \in \mathbf{H}^{1}(\Omega):\left.\mathbf{w}\right|_{\partial \Omega}=\mathbf{g}\right\} \times\left\{\boldsymbol{\Phi} \in \mathbf{H}^{1}(\Omega): \boldsymbol{\Phi} \times\left.\mathbf{n}\right|_{\partial \Omega}=\mathbf{q}\right\} \\
\text { and } p \in L_{0}^{2}(\Omega)
\end{gathered}
$$

such that

(5.4) $a((\mathbf{u}, \mathbf{B}),(\mathbf{u}, \mathbf{B}),(\mathbf{v}, \Psi))+b((\mathbf{v}, \Psi), p)=F((\mathbf{v}, \Psi)) \quad \forall(\mathbf{v}, \Psi) \in \mathscr{W}_{0 \tau}(\boldsymbol{\Omega})$

and

$$
b((\mathbf{u}, \mathbf{B}), \chi)=0 \quad \forall \chi \in L_{0}^{2}(\Omega) .
$$

In order to show that solutions of (5.3)-(5.5) are weak solutions of (2.17)-(2.21) and (5.1), i.e., that (5.3) $-(5.5)$ is a weak formulation of (2.17) $-(2.21)$ and (5.1), we proceed as in Proposition 3.1, with the following lemma replacing Lemma 3.2 .

Lemma 5.1. If $\mathbf{B} \in \mathbf{H}^{1}(\Omega)$, there exists a scalar $b \in H^{2}(\Omega)$ such that

$$
\nabla \cdot \nabla b=\nabla \cdot \mathbf{B} \text { and }\left.b\right|_{\partial \Omega}=0 \text {. }
$$

Moreover, $\nabla b \in \mathbf{H}_{\tau}^{1}(\Omega)$.

Proof. Since $\mathbf{B} \in \mathbf{H}^{1}(\Omega)$, we have that $\nabla \cdot \mathbf{B} \in L^{2}(\Omega)$. The result follows easily, since it is known that (5.6) has a solution $b \in H^{2}(\Omega)$ provided $\Omega$ is bounded and of class $C^{1,1}$, or $\Omega$ is a bounded convex polyhedron, $\Omega \subset \mathbb{R}^{3}$ (see [7] or [8]). Now since $\left.b\right|_{\partial \Omega}=0$, the tangential derivative of $b$ on the boundary vanishes, i.e., $\left.(\nabla b \times \mathbf{n})\right|_{\partial \Omega}=0$ (see [7]), and thus we have that $\nabla b \in \mathbf{H}_{\tau}^{1}(\Omega)$.

In order to derive existence and uniqueness results, we again proceed as in $\S 4$, beginning with the splitting $\mathbf{u}=\hat{\mathbf{u}}+\mathbf{u}_{0}$, where $\mathbf{u}_{0}$ satisfies $(4.1)$ and $\hat{\mathbf{u}} \in \mathbf{H}_{0}^{1}(\Omega)$. We also let $\mathbf{B}_{0}$ satisfy

$$
\begin{array}{ll}
\mathbf{B}_{0} \in \mathbf{H}^{1}(\boldsymbol{\Omega}), & \nabla \cdot \mathbf{B}_{0}=0, \quad \nabla \times \mathbf{B}_{0}=0, \\
\left.\left(\mathbf{B}_{0} \times \mathbf{n}\right)\right|_{\partial \Omega}=\mathbf{q}, & \left\|\mathbf{B}_{0}\right\|_{1} \leq \gamma_{2}\|\mathbf{q}\|_{1 / 2, \partial \Omega}
\end{array}
$$

for some positive constant $\gamma_{2}<\infty$. We then set $\mathbf{B}=\widehat{\mathbf{B}}+\mathbf{B}_{0}$, so that $\widehat{\mathbf{B}} \epsilon$ $\mathbf{H}_{\tau}^{1}(\Omega)$. Finding $\mathbf{B}_{0}$ satisfying (5.7) is more problematical than the analogous problem for the first set of boundary conditions; i.e., having $\mathbf{B}_{0}$ satisfy (4.2). In the general case, $\mathbf{q}$ must satisfy some additional compatibility conditions. Assume that, in addition to (5.1), $\mathbf{q}$ satisfies $\mathbf{n} \times \mathbf{q} \in \mathbf{H}^{1 / 2}(\partial \Omega)$; obviously $(\mathbf{n} \times \mathbf{q}) \cdot \mathbf{n}=0$, and thus

$$
\int_{\partial \Omega}(\mathbf{n} \times \mathbf{q}) \cdot \mathbf{n} d \mathbf{x}=0 .
$$

Hence, there exists a function $\mathbf{B}_{1} \in \mathbf{H}^{1}(\Omega)$ with

$$
\nabla \cdot \mathbf{B}_{1}=0,\left.\quad \mathbf{B}_{1}\right|_{\partial \Omega}=\mathbf{n} \times \mathbf{q}, \quad\left\|\mathbf{B}_{1}\right\|_{1} \leq C\|\mathbf{n} \times \mathbf{q}\|_{1 / 2, \partial \Omega} .
$$

Note that

$$
\left.\left(\mathbf{B}_{1} \times \mathbf{n}\right)\right|_{\partial \Omega}=(\mathbf{n} \times \mathbf{q}) \times \mathbf{n}=\mathbf{q}-(\mathbf{q} \cdot \mathbf{n}) \mathbf{n}=\mathbf{q},
$$


because of the compatibility condition (5.1) on $\mathbf{q}$, i.e., $\mathbf{q} \cdot \mathbf{n}=0$. If in addition,

$$
\left.\int_{\partial \Omega} \mathbf{q} \cdot \nabla \phi\right|_{\partial \Omega} d \mathbf{x}=0 \quad \forall \phi \in H^{2}(\Omega),
$$

we get that $\left.\left[\left(\nabla \times \mathbf{B}_{1}\right) \cdot \mathbf{n}\right]\right|_{\partial \boldsymbol{\Omega}}=0$, so there exists (see [7]) $\mathbf{B}_{2} \in \mathbf{H}_{\tau}^{1}(\boldsymbol{\Omega})$ such that

$$
\nabla \times \mathbf{B}_{2}=\nabla \times \mathbf{B}_{1}, \quad \nabla \cdot \mathbf{B}_{2}=0, \quad\left\|\mathbf{B}_{2}\right\|_{1} \leq C\left\|\mathbf{B}_{1}\right\|_{1} .
$$

Setting $\mathbf{B}_{0}=\mathbf{B}_{1}-\mathbf{B}_{2},(5.7)$ is clearly satisfied.

Now the rest of the analyses for the existence and uniqueness continues similarly to the one for the other boundary conditions (see $\S 4$ ); the major differences are replacing the space $\mathbf{H}_{n}^{1}(\Omega)$ by $\mathbf{H}_{\tau}^{1}(\Omega)$ and now defining $\widehat{F}((\mathbf{v}, \Psi))=$ $\langle\mathbf{f}, \mathbf{v}\rangle_{\Omega}-a\left(\left(\mathbf{u}_{0}, \mathbf{B}_{0}\right),\left(\mathbf{u}_{0}, \mathbf{B}_{0}\right),(\mathbf{v}, \Psi)\right)$. Of course, the exact form of the condition (4.26) for uniqueness and of the bounds (4.22) and (4.23) will change to reflect the different nature of the data for the problem.

Note that if the boundary is smooth, e.g., $C^{2}$, then slightly stronger results may be obtained by a vector potential formulation of $\mathbf{B}_{0}$.

If the additional condition (5.8) does not hold, in general there does not exist a $\mathbf{B}_{0}$ with $\nabla \times \mathbf{B}_{0}=0$ which satisfies the boundary conditions. However, one can still carry out the analyses if the data $\mathbf{q}$ is "sufficiently small". Indeed, one can always find a $\mathbf{B}_{0} \in \mathbf{H}^{1}(\Omega)$ such that $\nabla \cdot \mathbf{B}_{0}=0$ in $\Omega,\left.\left(\mathbf{B}_{0} \times \mathbf{n}\right)\right|_{\partial \Omega}=\mathbf{q}$, and $\left\|\mathbf{B}_{0}\right\|_{1} \leq \gamma_{2}\|\mathbf{q}\|_{1 / 2, \partial \Omega}$, where, of course, we require $\mathbf{q} \cdot \mathbf{n}=0$ on $\partial \Omega$. Then, if $\mathbf{q}$ is small, so will $\nabla \times \mathbf{B}_{0}$ be small, and again a lemma analogous to Lemma 4.4 can be proved. The subsequent analyses proceed as in $\S 4$.

\section{APPROXIMATION}

6.1. The approximate problem. We now want to consider approximating solutions to our problem. In order to keep the exposition simple, we restrict our attention to convex polyhedral domains. We start by choosing families of finitedimensional spaces $\mathbf{X}^{h}(\Omega) \subset \mathbf{H}^{1}(\Omega), \mathbf{Y}^{h}(\Omega) \subset \mathbf{H}^{1}(\Omega)$, and $S_{0}^{h}(\Omega) \subset L_{0}^{2}(\Omega)$, parametrized by a parameter $h$ such that $0<h<1$. We then define

$$
\begin{gathered}
\mathbf{X}_{0}^{h}(\Omega):=\mathbf{X}^{h}(\Omega) \cap \mathbf{H}_{0}^{1}(\Omega), \quad \mathbf{Y}_{n}^{h}(\Omega):=\mathbf{Y}^{h}(\Omega) \cap \mathbf{H}_{n}^{1}(\Omega), \\
\mathbf{Y}_{\tau}^{h}(\Omega):=\mathbf{Y}^{h}(\Omega) \cap \mathbf{H}_{\tau}^{1}(\Omega),
\end{gathered}
$$

with corresponding norms induced by the norms on $\mathbf{H}^{1}(\Omega)$ and $L_{0}^{2}(\Omega)$. Next, we define the product spaces

$$
\begin{aligned}
& \mathscr{W}^{h}(\Omega):=\mathbf{X}^{h}(\Omega) \times \mathbf{Y}^{h}(\Omega), \\
& \mathscr{W}_{0 n}^{h}(\Omega):=\mathbf{X}_{0}^{h}(\Omega) \times \mathbf{Y}_{n}^{h}(\Omega), \\
& \mathscr{W}_{0 \tau}^{h}(\Omega):=\mathbf{X}_{0}^{h}(\Omega) \times \mathbf{Y}_{\tau}^{h}(\Omega),
\end{aligned}
$$

with corresponding norms induced by the norm on $\mathscr{W}(\Omega)$. We also define the space

$$
\mathbf{Z}^{h}(\Omega):=\left\{\mathbf{w}^{h} \in \mathbf{X}_{0}^{h}(\Omega): \int_{\Omega} \chi^{h} \nabla \cdot \mathbf{w}^{h} d \mathbf{x}=0 \forall \chi^{h} \in S_{0}^{h}(\Omega)\right\}
$$


Note that, in general, $\mathbf{Z}^{h}(\Omega) \not \subset \mathbf{Z}(\Omega)$. A measure of the "angle" between the spaces $\mathbf{Z}(\Omega)$ and $\mathbf{Z}^{h}(\Omega)$ (see $[9,13]$ ) is given by

$$
\Theta:=\sup _{\substack{\mathbf{z}^{h} \in \mathbf{Z}^{h}(\Omega) \\\left\|\mathbf{z}^{h}\right\|_{1}=1}} \inf _{\mathbf{z} \in \mathbf{Z}(\Omega)}\left\|\mathbf{z}-\mathbf{z}^{h}\right\|_{1} .
$$

Note that $0 \leq \Theta \leq 1$. A consequence of $\mathbf{Z}^{h}(\Omega) \not \subset \mathbf{Z}(\Omega)$ is that the form $a_{1}(\cdot, \cdot, \cdot)$ defined by $(3.2)$ does not satisfy the antisymmetry property (4.11) whenever $\mathbf{u} \in \mathbf{Z}^{h}(\Omega)$. In order to preserve this useful antisymmetry property over the subspaces, we introduce the form (see [6] or [17])

$$
\begin{aligned}
\tilde{a}_{1}((\mathbf{u}, \mathbf{B}),(\mathbf{v}, \boldsymbol{\Psi}),(\mathbf{w}, \boldsymbol{\Phi})) \\
:=\int_{\Omega} \frac{1}{2 N}[\mathbf{u} \cdot \nabla \mathbf{v} \cdot \mathbf{w}-\mathbf{u} \cdot \nabla \mathbf{w} \cdot \mathbf{v}] d \mathbf{x} \\
\quad-\int_{\Omega} \frac{1}{R_{m}}[(\nabla \times \Psi) \times \mathbf{B} \cdot \mathbf{w}-(\nabla \times \boldsymbol{\Phi}) \times \mathbf{B} \cdot \mathbf{v}] d \mathbf{x} \\
=\frac{1}{2}\left[a_{1}((\mathbf{u}, \mathbf{B}),(\mathbf{v}, \boldsymbol{\Psi}),(\mathbf{w}, \boldsymbol{\Phi}))-a_{1}((\mathbf{u}, \mathbf{B}),(\mathbf{w}, \boldsymbol{\Phi}),(\mathbf{v}, \Psi))\right] .
\end{aligned}
$$

Using the divergence theorem, it is easily verified that

$$
\tilde{a}_{1}((\mathbf{u}, \mathbf{B}),(\mathbf{v}, \Psi),(\mathbf{w}, \boldsymbol{\Phi}))=a_{1}((\mathbf{u}, \mathbf{B}),(\mathbf{v}, \Psi),(\mathbf{w}, \boldsymbol{\Phi}))
$$

on $\left(\mathbf{Z}(\Omega) \times \mathbf{H}^{1}(\Omega)\right) \times \mathscr{W}(\Omega) \times \mathscr{W}(\Omega)$. In addition, we now have that

$$
\tilde{a}_{1}((\mathbf{u}, \mathbf{B}),(\mathbf{v}, \Psi),(\mathbf{w}, \boldsymbol{\Phi}))=-\tilde{a}_{1}((\mathbf{u}, \mathbf{B}),(\mathbf{w}, \boldsymbol{\Phi}),(\mathbf{v}, \Psi))
$$

on all of $\mathscr{W}(\Omega) \times \mathscr{W}(\Omega) \times \mathscr{W}(\Omega)$, and in particular, this antisymmetry property holds on the finite-dimensional subspaces, e.g., even when $\mathbf{u} \in \mathbf{X}^{h}(\Omega)$.

We now modify the form $a(\cdot, \cdot, \cdot)$ defined in (3.5) into the form $\tilde{a}(\cdot, \cdot, \cdot)$ defined by

$$
\begin{aligned}
\tilde{a}((\mathbf{u}, \mathbf{B}),(\mathbf{v}, \Psi),(\mathbf{w}, \boldsymbol{\Phi})):= & a_{0}((\mathbf{v}, \Psi),(\mathbf{w}, \boldsymbol{\Phi})) \\
& +\tilde{a}_{1}((\mathbf{u}, \mathbf{B}),(\mathbf{v}, \Psi),(\mathbf{w}, \boldsymbol{\Phi})) .
\end{aligned}
$$

Owing to (3.8) (respectively, (5.5)) and (6.2), it makes no difference whether one uses $a(\cdot, \cdot, \cdot)$ or $\tilde{a}(\cdot, \cdot, \cdot)$ in (3.7) (respectively, (5.4)). In fact, all of the results of $\S \S 3-5$ remain valid if one uses, throughout those sections, $\tilde{a}_{1}(\cdot, \cdot, \cdot)$ and $\tilde{a}(\cdot, \cdot, \cdot)$ instead of $a_{1}(\cdot, \cdot, \cdot)$ and $a(\cdot, \cdot, \cdot)$.

Next, we approximate $\mathbf{g}$ and $q$ or $\mathbf{q}$ by $\mathbf{g}^{h}$ and $q^{h}$ or $\mathbf{q}^{h}$, which belong to the restriction to the boundary of elements of $\mathbf{X}^{h}(\Omega)$, to the restriction to the boundary of normal components of $\mathbf{Y}^{h}(\Omega)$, and to the restriction to the boundary of tangential components of $\mathbf{Y}^{h}(\Omega)$, respectively. There are various ways to choose these approximations to the boundary conditions. For example, they may be chosen to be the interpolants in the boundary spaces of the corresponding functions, or they may be chosen to be some projection of the given data onto the boundary spaces. For now, we assume that we have available approximations $\left.\mathbf{g}^{h} \in \mathbf{X}^{h}(\Omega)\right|_{\partial \Omega}$ and $q^{h} \in\left\{\left.\left(\mathbf{v}^{h} \cdot \mathbf{n}\right)\right|_{\partial \Omega}: \mathbf{v}^{h} \in \mathbf{Y}^{h}(\boldsymbol{\Omega})\right\}$ or 
$\mathbf{q}^{h} \in\left\{\left.\left(\mathbf{n} \times \mathbf{v}^{h}\right)\right|_{\partial \boldsymbol{\Omega}}: \mathbf{v}^{h} \in \mathbf{Y}^{h}(\boldsymbol{\Omega})\right\}$ to $\mathbf{g}$ and $q$ or $\mathbf{q}$, respectively. Note that for polyhedral domains, $\mathbf{g}^{h}, q^{h}$, and $\mathbf{q}^{h}$ are piecewise polynomials on the boundary whenever the underlying finite element spaces $\mathbf{X}^{h}(\Omega)$ and $\mathbf{Y}^{h}(\Omega)$ are themselves piecewise polynomial spaces. (This is due to the fact that for polyhedral domains the components of the normal to the boundary are piecewise constant functions.)

The discrete problem we consider, for the boundary conditions $(2.8)-(2.10)$, is given as follows: find

$$
\begin{gathered}
\left(\mathbf{u}^{h}, \mathbf{B}^{h}\right) \in\left\{\mathbf{v}^{h} \in \mathbf{X}^{h}(\Omega):\left.\mathbf{v}^{h}\right|_{\partial \Omega}=\mathbf{g}^{h}\right\} \times\left\{\boldsymbol{\Phi}^{h} \in \mathbf{Y}^{\dot{h}}(\Omega):\left.\left(\boldsymbol{\Phi}^{h} \cdot \mathbf{n}\right)\right|_{\partial \Omega}=q^{h}\right\} \\
\text { and } p^{h} \in S_{0}^{h}(\Omega)
\end{gathered}
$$

such that

$$
\begin{gathered}
\tilde{a}\left(\left(\mathbf{u}^{h}, \mathbf{B}^{h}\right),\left(\mathbf{u}^{h}, \mathbf{B}^{h}\right),\left(\mathbf{v}^{h}, \Psi^{h}\right)\right)+b\left(\left(\mathbf{v}^{h}, \Psi^{h}\right), p^{h}\right) \\
=F\left(\left(\mathbf{v}^{h}, \Psi^{h}\right)\right) \quad \forall\left(\mathbf{v}^{h}, \Psi^{h}\right) \in \mathscr{W}_{0 n}^{h}(\Omega), \\
b\left(\left(\mathbf{u}^{h}, \mathbf{B}^{h}\right), \chi^{h}\right)=0 \quad \forall \chi^{h} \in S_{0}^{h}(\Omega),
\end{gathered}
$$

where $F(\cdot)$ and $b(\cdot, \cdot)$ are defined by (3.4) and (3.3), respectively.

For the boundary conditions (2.8), (2.11), and (2.12), the approximate problem is given as follows: find

$$
\begin{gathered}
\left(\mathbf{u}^{h}, \mathbf{B}^{h}\right) \in\left\{\mathbf{v}^{h} \in \mathbf{X}^{h}(\Omega):\left.\mathbf{v}^{h}\right|_{\partial \Omega}=\mathbf{g}^{h}\right\} \times\left\{\boldsymbol{\Phi}^{h} \in \mathbf{Y}^{h}(\Omega):\left.\left(\boldsymbol{\Phi}^{h} \times \mathbf{n}\right)\right|_{\partial \Omega}=\mathbf{q}^{h}\right\} \\
\text { and } p^{h} \in S_{0}^{h}(\Omega),
\end{gathered}
$$

such that (6.6) is satisfied for all $\left(\mathbf{v}^{h}, \Psi^{h}\right) \in \mathscr{W}_{0 \tau}^{h}(\Omega)$ and (6.7) is satisfied, where $F(\cdot)$ is now defined by $(5.2)$.

6.2. Existence and uniqueness results for the approximate problem. Let us consider the discrete problem given by (6.5)-(6.7) corresponding to the boundary conditions $(2.8)-(2.10)$. The derivation of existence and uniqueness results for this problem closely mimics that of $\S 4$ for the continuous problem (3.6)-(3.8).

We begin by restating, in the present context, Lemmas 4.1 and 4.3 , and part of Lemma 4.2.

Lemma 6.1. The bilinear forms $a_{0}(\cdot, \cdot)$ and $b(\cdot, \cdot)$, the trilinear form $\tilde{a}_{1}(\cdot, \cdot, \cdot)$, and the linear functional $F(\cdot)$ are continuous on $\mathscr{W}^{h}(\Omega) \times \mathscr{W}^{h}(\Omega), \mathscr{W}^{h}(\Omega) \times$ $S_{0}^{h}(\Omega), \mathscr{W}^{h}(\Omega) \times \mathscr{W}^{h}(\Omega) \times \mathscr{W}^{h}(\Omega)$, and $\mathbf{X}_{0}^{h}(\Omega) \times \mathbf{Y}^{h}(\Omega)$, respectively. Furthermore, the form $a_{0}(\cdot, \cdot)$ is coercive on $\mathscr{W}_{0 n}^{h}(\Omega) \times \mathscr{W}_{0 n}^{h}(\Omega)$ and, on $\mathscr{W}^{h}(\Omega) \times$ $\mathscr{W}^{h}(\Omega) \times \mathscr{W}^{h}(\Omega)$, the form $\tilde{a}_{1}(\cdot, \cdot, \cdot)$ is antisymmetric in its last two arguments, i.e., (6.3) is satisfied.

Proof. The continuity properties are an obvious consequence of Lemma 4.1 and, for $\tilde{a}_{1}(\cdot, \cdot, \cdot)$, of $(6.1)$ and Lemma 4.1. Since $\mathscr{W}_{0 n}^{h}(\Omega) \subset \mathscr{W}_{0 n}(\Omega)$, the coercivity result for $a_{0}(\cdot, \cdot)$ is an obvious consequence of Lemma 4.2. The antisymmetry property $(6.3)$ is satisfied by construction. 
The constants appearing in (4.5)-(4.8) and (4.10) carry over to the present setting.

The inf-sup condition $(4.9)$ for $b(\cdot, \cdot)$ is not automatically satisfied over the subspaces $\mathscr{W}_{0 n}^{h}(\Omega), S_{0}^{h}(\Omega)$. This condition turns out, in fact, to be a constraint on the finite-dimensional subspaces $\mathbf{X}_{0}^{h}(\Omega)$ and $S_{0}^{h}(\Omega)$ that can be used in order to have stable and accurate approximations. Thus, we assume that $\mathbf{X}_{0}^{h}(\Omega)$ and $S_{0}^{h}(\Omega)$ have been chosen so that the inf-sup condition is satisfied on the finite-dimensional spaces; i.e., that there exists a constant $\beta^{h}>0$ such that

$$
\inf _{\chi^{h} \in S_{0}^{h}(\Omega)} \sup _{\left(\mathbf{u}^{h}, \mathbf{B}^{h}\right) \in \mathscr{W}_{0}^{h}(\Omega)} \frac{b\left(\left(\mathbf{u}^{h}, \mathbf{B}^{h}\right), \chi^{h}\right)}{\left\|\left(\mathbf{u}^{h}, \mathbf{B}^{h}\right)\right\|_{\mathscr{W}}\left\|\chi^{h}\right\|_{0}} \geq \beta^{h} .
$$

This constraint is exactly the one necessary for the analogous discretization of the Navier-Stokes equations to yield meaningful approximations. Thus, a variety of pairs of finite element spaces that satisfy (6.9) have been devised and analyzed; see the discussion and references in $[7,9,16]$. There is no constraint on the spaces $\mathbf{Y}_{n}^{h}(\Omega)$, so in order to approximate the velocity and pressure, we can use the spaces that have been used traditionally for the Navier-Stokes equations; to approximate the magnetic field we can use any appropriate subspace of $\mathbf{H}^{1}(\Omega)$. When guided by the error estimates derived below, it is convenient and efficient to choose $\mathbf{Y}^{h}(\Omega)=\mathbf{X}^{h}(\Omega)$, i.e., the underlying finite element spaces for the magnetic and velocity fields are the same.

Next, we need to define extensions $\mathbf{u}_{0}^{h} \in \mathbf{X}^{h}(\Omega)$ and $\mathbf{B}_{0}^{h} \in \mathbf{Y}^{h}(\Omega)$ of the discrete data $\mathbf{g}^{h}$ and $q^{h}$, respectively. We emphasize at the outset that these extensions are used only to derive existence and uniqueness results, and are not explicitly needed in order to compute the solution of the approximate problem (6.5)-(6.7). We want these extensions to satisfy, on these subspaces, relations analogous to (4.1) and (4.2).

Given $\mathbf{g}^{h}$, we seek $\mathbf{u}_{0}^{h} \in \mathbf{X}^{h}(\Omega)$ such that $\left.\mathbf{u}_{0}^{h}\right|_{\partial \Omega}=\mathbf{g}^{h}$,

$$
\int_{\Omega}\left(\nabla \cdot \mathbf{u}_{0}^{h}\right) \chi^{h} d \mathbf{x}=0 \quad \forall \chi^{h} \in S_{0}^{h}(\Omega)
$$

and $\left\|\mathbf{u}_{0}^{h}\right\|_{1} \leq \gamma_{1}^{h}\left\|\mathbf{g}^{h}\right\|_{1 / 2, \partial \Omega}$. The existence of such a $\mathbf{u}_{0}^{h}$ can be demonstrated as follows. First, given $\left.\mathbf{g}^{h} \in \mathbf{X}^{h}(\Omega)\right|_{\partial \Omega} \subset \mathbf{H}^{1 / 2}(\partial \Omega)$, we can easily find a $\mathbf{u}_{1}^{h} \in$ $\mathbf{X}^{h}(\Omega)$ such that $\left.\mathbf{u}_{1}^{h}\right|_{\partial \Omega}=\mathbf{g}^{h}$ and $\left\|\mathbf{u}_{1}^{h}\right\|_{1} \leq C\left\|\mathbf{g}^{h}\right\|_{1 / 2, \partial \Omega}$. Then, we let $\mathbf{u}_{2}^{h} \in$ $\mathbf{X}_{0}^{h}(\Omega)$ satisfy

$$
\int_{\Omega}\left(\nabla \cdot \mathbf{u}_{2}^{h}\right) \chi^{h} d \mathbf{x}=-\int_{\Omega}\left(\nabla \cdot \mathbf{u}_{1}^{h}\right) \chi^{h} d \mathbf{x} \quad \forall \chi^{h} \in S_{0}^{h}(\Omega) .
$$

The existence of such a $\mathbf{u}_{2}^{h}$ follows from (4.7) and (6.9); moreover, these also imply that there exists a $\mathbf{u}_{2}^{h}$ such that $\left\|\mathbf{u}_{2}^{h}\right\|_{1} \leq\left(1 / \beta^{h}\right)\left\|\nabla \cdot \mathbf{u}_{1}^{h}\right\|_{0} \leq\left(\sqrt{3} / \beta^{h}\right)\left\|\mathbf{u}_{1}^{h}\right\|_{1} \leq$ $\left(\sqrt{3} C / \beta^{h}\right)\left\|\mathbf{g}^{h}\right\|_{1 / 2, \partial \Omega}$. Then $\mathbf{u}_{0}^{h}:=\mathbf{u}_{1}^{h}+\mathbf{u}_{2}^{h}$ has the desired properties, including (6.10). 
To construct an extension $\mathbf{B}_{0}^{h}$, we proceed as follows. Given $q^{h}$, we find $\mathbf{B}_{0}^{h} \in \mathbf{Y}^{h}(\boldsymbol{\Omega})$ that satisfies $\left.\left(\mathbf{B}_{0}^{h} \cdot \mathbf{n}\right)\right|_{\partial \boldsymbol{\Omega}}=q^{h}$ and

$$
\int_{\Omega}\left[\left(\nabla \times \mathbf{B}_{0}^{h}\right) \cdot\left(\nabla \times \Psi^{h}\right)+\left(\nabla \cdot \mathbf{B}_{0}^{h}\right)\left(\nabla \cdot \Psi^{h}\right)\right] d \mathbf{x}=0 \quad \forall \Psi^{h} \in \mathbf{Y}_{n}^{h}(\Omega) .
$$

It is not difficult to show, using the methods of [5], that such a $\mathbf{B}_{0}^{h}$ exists and moreover, that $\left\|\mathbf{B}_{0}^{h}\right\|_{1} \leq \gamma_{2}^{h}\left\|q^{h}\right\|_{1 / 2, \partial \Omega}$ and

$$
\left\|\mathbf{B}_{0}-\mathbf{B}_{0}^{h}\right\|_{1} \leq C \inf _{\substack{\left.\Phi^{h} \in \mathbf{Y}^{h}(\Omega) \\\left(\boldsymbol{\Phi}^{h} \cdot \mathbf{n}\right)\right|_{\partial \boldsymbol{\Omega}}=q^{h}}}\left\|\mathbf{B}_{0}-\boldsymbol{\Phi}^{h}\right\|_{1},
$$

where $\mathbf{B}_{0}$ is defined in (4.2). It can also be shown, again using the methods of [5], that if $q^{h}$ is chosen as either the interpolant or the $L^{2}$-projection of $q$ in the restriction of the normal components of $\mathbf{Y}^{h}(\Omega)$ to the boundary $\partial \Omega$, then the right-hand side of (6.11) can be made as small as one wishes by choosing $h$ sufficiently small. Thus, (4.2) and (6.11) imply that $\left\|\nabla \times \mathbf{B}_{0}^{h}\right\|_{0}$ and $\left\|\nabla \cdot \mathbf{B}_{0}^{h}\right\|_{0}$ can be made arbitrarily small as well.

To summarize, we have constructed extensions $\mathbf{u}_{0}^{h}$ and $\mathbf{B}_{0}^{h}$ of the data $\mathbf{g}^{h}$ and $q^{h}$, respectively, which satisfy, for any $\varepsilon>0$ and for sufficiently small $h$, (6.10),

$$
\mathbf{u}_{0}^{h} \in \mathbf{X}^{h}(\Omega),\left.\quad \mathbf{u}_{0}^{h}\right|_{\partial \Omega}=\mathbf{g}^{h}, \quad\left\|\mathbf{u}_{0}^{h}\right\|_{1} \leq \gamma_{1}^{h}\left\|\mathbf{g}^{h}\right\|_{1 / 2, \partial \Omega},
$$

and

$$
\begin{gathered}
\mathbf{B}_{0}^{h} \in \mathbf{Y}^{h}(\Omega),\left.\quad\left(\mathbf{B}_{0}^{h} \cdot \mathbf{n}\right)\right|_{\partial \Omega}=q^{h}, \quad\left\|\mathbf{B}_{0}^{h}\right\|_{1} \leq \gamma_{2}^{h}\left\|q^{h}\right\|_{1 / 2, \partial \Omega}, \\
\left\|\nabla \times \mathbf{B}_{0}^{h}\right\|_{0} \leq \varepsilon, \quad \text { and }\left\|\nabla \cdot \mathbf{B}_{0}^{h}\right\|_{0} \leq \varepsilon .
\end{gathered}
$$

It should be noted that the above construction processes also yield that $\gamma_{1}^{h}$ and $\gamma_{2}^{h}$ may be bounded from above uniformly in $h$ whenever $\beta^{h}$ can be bounded from below uniformly in $h$. Indeed, we may essentially take $\gamma_{1}^{h}=\gamma_{1}$ and $\gamma_{2}^{h}=\gamma_{2}$.

Having constructed the extensions $\mathbf{u}_{0}^{h}$ and $\mathbf{B}_{0}^{h}$, we proceed as in $\S 4$. We let $\mathbf{u}^{h}=\mathbf{u}_{0}^{h}+\hat{\mathbf{u}}^{h}$ and $\mathbf{B}^{h}=\mathbf{B}_{0}^{h}+\widehat{\mathbf{B}}^{h}$, so that $\hat{\mathbf{u}}^{h} \in \mathbf{X}_{0}^{h}(\Omega)$ and $\widehat{\mathbf{B}}^{h} \in \mathbf{Y}_{n}^{h}(\Omega)$. In view of (6.12) and (6.13), this leads us to the following discrete problem with homogeneous boundary conditions: find $\left(\hat{\mathbf{u}}^{h}, \widehat{\mathbf{B}}^{h}\right) \in \mathscr{W}_{0 n}^{h}(\Omega)$ and $p^{h} \in S_{0}^{h}(\Omega)$ such that

$$
\begin{gathered}
\hat{a}_{h}\left(\left(\hat{\mathbf{u}}^{h}, \widehat{\mathbf{B}}^{h}\right),\left(\hat{\mathbf{u}}^{h}, \widehat{\mathbf{B}}^{h}\right),\left(\mathbf{v}^{h}, \Psi^{h}\right)\right)+b\left(\left(\mathbf{v}^{h}, \Psi^{h}\right), p^{h}\right) \\
=\widehat{F}_{h}\left(\left(\mathbf{v}^{h}, \Psi^{h}\right)\right) \quad \forall\left(\mathbf{v}^{h}, \Psi^{h}\right) \in \mathscr{W}_{0 n}^{h}(\boldsymbol{\Omega})
\end{gathered}
$$

and

$$
b\left(\left(\hat{\mathbf{u}}^{h}, \widehat{\mathbf{B}}^{h}\right), \chi^{h}\right)=0 \quad \forall \chi^{h} \in S_{0}^{h}(\Omega),
$$


where

$$
\widehat{F}_{h}\left(\left(\mathbf{v}^{h}, \Psi^{h}\right)\right):=F\left(\left(\mathbf{v}^{h}, \Psi^{h}\right)\right)-\tilde{a}\left(\left(\mathbf{u}_{0}^{h}, \mathbf{B}_{0}^{h}\right),\left(\mathbf{u}_{0}^{h}, \mathbf{B}_{0}^{h}\right),\left(\mathbf{v}^{h}, \Psi^{h}\right)\right)
$$

and

$$
\begin{aligned}
\hat{a}_{h}\left(\left(\mathbf{u}^{h}, \mathbf{B}^{h}\right),\left(\mathbf{v}^{h}, \Psi^{h}\right),\left(\mathbf{w}^{h}, \boldsymbol{\Phi}^{h}\right)\right):= & \tilde{a}\left(\left(\mathbf{u}^{h}, \mathbf{B}^{h}\right),\left(\mathbf{v}^{h}, \Psi^{h}\right),\left(\mathbf{w}^{h}, \boldsymbol{\Phi}^{h}\right)\right) \\
& +\tilde{a}_{1}\left(\left(\mathbf{v}^{h}, \Psi^{h}\right),\left(\mathbf{u}_{0}^{h}, \mathbf{B}_{0}^{h}\right),\left(\mathbf{w}^{h}, \boldsymbol{\Phi}^{h}\right)\right) \\
& +\tilde{a}_{1}\left(\left(\mathbf{u}_{0}^{h}, \mathbf{B}_{0}^{h}\right),\left(\mathbf{v}^{h}, \Psi^{h}\right),\left(\mathbf{w}^{h}, \boldsymbol{\Phi}^{h}\right)\right) .
\end{aligned}
$$

We are now in a position to state results for the discrete problem analogous to those found in Lemmas 4.4-4.5 for the continuous problem.

Lemma 6.2. For sufficiently small $h$, there exists a constant $\alpha^{h}>0$ such that whenever

$$
\left\|\mathbf{g}^{h}\right\|_{1 / 2, \partial \Omega}<\frac{1}{\gamma_{1}^{h} \gamma_{3}} \min \left\{\frac{N k_{1}}{M^{2}}, \frac{k_{2}}{\sqrt{2} R_{m}}\right\} \text {, }
$$

then

$$
\begin{aligned}
& \hat{a}_{h}\left(\left(\mathbf{u}^{h}, \mathbf{B}^{h}\right),\left(\mathbf{u}^{h}, \mathbf{B}^{h}\right),\left(\mathbf{u}^{h}, \mathbf{B}^{h}\right)\right) \\
& \quad \geq \alpha^{h}\left\|\left(\mathbf{u}^{h}, \mathbf{B}^{h}\right)\right\|_{\mathscr{W}}^{2} \quad \forall\left(\mathbf{u}^{h}, \mathbf{B}^{h}\right) \in \mathbf{Z}^{h}(\Omega) \times \mathbf{Y}_{n}^{h}(\Omega) .
\end{aligned}
$$

Furthermore, the map

$$
\left(\mathbf{u}^{h}, \mathbf{B}^{h}\right) \in \mathbf{Z}^{h}(\Omega) \times \mathbf{Y}_{n}^{h}(\Omega) \rightarrow \hat{a}_{h}\left(\left(\mathbf{u}^{h}, \mathbf{B}^{h}\right),\left(\mathbf{u}^{h}, \mathbf{B}^{h}\right),\left(\mathbf{v}^{h}, \Psi^{h}\right)\right)
$$

is weakly sequentially continuous on $\mathbf{Z}^{h}(\Omega) \times \mathbf{Y}_{n}^{h}(\Omega)$.

Proof. The proof follows exactly the proofs of Lemmas 4.4 and 4.5 , the only exception being that since $\nabla \times \mathbf{B}_{0}^{h} \neq 0$, we must choose $h$ sufficiently small so that we can appropriately hide any terms involving $\nabla \times \mathbf{B}_{0}^{h}$.

Again, for reasonable choices of finite element spaces and for sufficiently small $h$, the constant $\alpha^{h}$ may be bounded independently of $h$ and, in fact, we may essentially choose $\alpha^{h}=\alpha$. Armed with Lemmas 6.1 and 6.2, we may now state an existence and uniqueness result for the solution of the discrete problem (6.5)-(6.7).

Theorem 6.3. Let $\left.\mathbf{g}^{h} \in \mathbf{X}^{h}(\Omega)\right|_{\partial \Omega}$ satisfy (6.14), $\mathbf{f}$ and $\mathbf{k}$ satisfy (2.13) and (2.16), and $q^{h} \in\left\{\left.\left(\Psi^{h} \cdot \mathbf{n}\right)\right|_{\partial \Omega}: \Psi^{h} \in \mathbf{Y}^{h}(\Omega)\right\}$, and assume (6.9) holds. Then there exists a solution $\left(\left(\mathbf{u}^{h}, \mathbf{B}^{h}\right), p^{h}\right) \in \mathscr{W}^{h}(\Omega) \times S_{0}^{h}(\Omega)$ of $(6.5)-(6.7)$. Moreover, the estimates (4.22) and (4.23) hold with $\mathbf{u}, \mathbf{B}, p, \mathbf{g}, q, \gamma_{1}, \gamma_{2}, \alpha$, and $\beta$ replaced by $\mathbf{u}^{h}, \mathbf{B}^{h}, p^{h}, \mathbf{g}^{h}, q^{h}, \gamma_{1}^{h}, \gamma_{2}^{h}, \alpha^{h}$, and $\beta^{h}$, respectively. Moreover, there is at most one solution satisfying (4.25) (with the above mentioned replacements). Finally, if (4.26) (again with the appropriate replacements) holds, then the problem (6.5)-(6.7) has exactly one solution.

Proof. The proof proceeds exactly as those for Theorems 4.6 and 4.7. 
A similar existence and uniqueness result may be derived for the solution of $(6.6)-(6.8),\left(\mathscr{W}_{0 n}^{h}(\Omega)\right.$ replaced by $\mathscr{W}_{0 \tau}^{h}(\Omega)$ in $\left.(6.6)\right)$, corresponding to the boundary conditions $(2.8),(2.11)$, and (2.12).

6.3. Error estimates. We now turn to the derivation of an estimate for the difference between the solution of the approximate problem (6.5)-(6.7) and of the continuous problem (3.6)-(3.8). Again, we consider in detail the case of the boundary conditions $(2.8)-(2.10)$. We also only treat the case where both the continuous and discrete problems have a unique solution. We define the set $\mathscr{W}_{1}^{h}(\Omega)$ by

$$
\mathscr{W}_{1}^{h}(\Omega):=\mathbf{W}_{1}^{h}(\Omega) \times \mathbf{Y}_{1}^{h}(\Omega)
$$

where

$$
\begin{gathered}
\mathbf{W}_{1}^{h}(\Omega):=\left\{\mathbf{w}^{h} \in \mathbf{X}^{h}(\Omega):\left.\mathbf{w}^{h}\right|_{\partial \Omega}=\mathbf{g}^{h}\right\}, \\
\mathbf{Y}_{1}^{h}(\Omega):=\left\{\boldsymbol{\Phi}^{h} \in \mathbf{Y}^{h}(\boldsymbol{\Omega}):\left.\left(\boldsymbol{\Phi}^{h} \cdot \mathbf{n}\right)\right|_{\partial \Omega}=q^{h}\right\} .
\end{gathered}
$$

Then the basic error estimate is given by the following theorem.

Theorem 6.4. Let $((\mathbf{u}, \mathbf{B}), p)$ and $\left(\left(\mathbf{u}^{h}, \mathbf{B}^{h}\right), p^{h}\right)$ denote the solutions of the problems (3.6)-(3.8) and (6.5)-(6.7), respectively. Let the hypotheses of Theorems 4.6, 4.7, and 6.3 be valid. Then, there exist positive constants $C_{i}<\infty$, $i=1, \ldots, 4$, such that

$$
\begin{aligned}
\left\|(\mathbf{u}, \mathbf{B})-\left(\mathbf{u}^{h}, \mathbf{B}^{h}\right)\right\|_{\mathscr{W}} \leq & C_{1} \inf _{\left(\mathbf{v}^{h}, \Psi^{h}\right) \in \mathscr{W}_{1}^{h}(\Omega)}\left\|(\mathbf{u}, \mathbf{B})-\left(\mathbf{v}^{h}, \Psi^{h}\right)\right\|_{\mathscr{W}} \\
& +C_{2} \Theta \inf _{s^{h} \in S_{0}^{h}(\Omega)}\left\|p-s^{h}\right\|_{0}
\end{aligned}
$$

and

(6.16) $\left\|p-p^{h}\right\|_{0} \leq C_{3} \inf _{\left(\mathbf{v}^{h}, \Psi^{h}\right) \in \mathscr{W}_{1}^{h}(\Omega)}\left\|(\mathbf{u}, \mathbf{B})-\left(\mathbf{v}^{h}, \Psi^{h}\right)\right\|_{\mathscr{W}}+C_{4} \inf _{s^{h} \in S_{0}^{h}(\Omega)}\left\|p-s^{h}\right\|_{0}$.

Proof. Let $\left(\mathbf{w}^{h}, \boldsymbol{\Phi}^{h}\right)$ be an arbitrary element of $\mathscr{W}_{2}^{h}(\Omega):=\mathbf{W}_{2}^{h}(\Omega) \times \mathbf{Y}_{1}^{h}(\Omega)$, where

$$
\mathbf{W}_{2}^{h}(\Omega):=\left\{\mathbf{w}^{h} \in \mathbf{W}_{1}^{h}(\Omega): b\left(\left(\mathbf{w}^{h}, \boldsymbol{\Phi}^{h}\right), \chi^{h}\right)=0 \forall \chi^{h} \in S_{0}^{h}(\Omega)\right\} .
$$

Then clearly, $\left(\mathbf{u}^{h}-\mathbf{w}^{h}, \mathbf{B}^{h}-\boldsymbol{\Phi}^{h}\right) \in \mathscr{W}_{0 n}^{h}(\Omega)$ and, in fact, since

$$
\begin{aligned}
b\left(\left(\mathbf{u}^{h}-\mathbf{w}^{h}, \mathbf{B}^{h}-\boldsymbol{\Phi}^{h}\right), \chi^{h}\right) & =b\left(\left(\mathbf{u}^{h}, \mathbf{B}^{h}\right), \chi^{h}\right)-b\left(\left(\mathbf{w}^{h}, \boldsymbol{\Phi}^{h}\right), \chi^{h}\right) \\
& =0 \quad \forall \chi^{h} \in S_{0}^{h}(\Omega),
\end{aligned}
$$

we have that $\left(\mathbf{u}^{h}-\mathbf{w}^{h}, \mathbf{B}^{h}-\boldsymbol{\Phi}^{h}\right) \in \mathbf{Z}^{h}(\Omega) \times \mathbf{Y}_{n}^{h}(\Omega)$. For the exact solution $(\mathbf{u}, \mathbf{B})$ we also have, from (3.7), (6.2), and (6.4), that

$$
\begin{aligned}
& a_{0}\left((\mathbf{u}, \mathbf{B}),\left(\mathbf{v}^{h}, \Psi^{h}\right)\right)+\tilde{a}_{1}\left((\mathbf{u}, \mathbf{B}),(\mathbf{u}, \mathbf{B}),\left(\mathbf{v}^{h}, \Psi^{h}\right)\right)+b\left(\left(\mathbf{v}^{h}, \Psi^{h}\right), p\right) \\
& =F\left(\left(\mathbf{v}^{h}, \Psi^{h}\right)\right) \quad \forall\left(\mathbf{v}^{h}, \Psi^{h}\right) \in \mathscr{W}_{0 n}^{h}(\Omega) .
\end{aligned}
$$


For the approximate solution $\left(\mathbf{u}^{h}, \mathbf{B}^{h}\right)$ we have, from (6.4) and (6.6),

$$
\begin{aligned}
a_{0}\left(\left(\mathbf{u}^{h}, \mathbf{B}^{h}\right),\left(\mathbf{v}^{h}, \Psi^{h}\right)\right)+\tilde{a}_{1}\left(\left(\mathbf{u}^{h}, \mathbf{B}^{h}\right),\left(\mathbf{u}^{h}, \mathbf{B}^{h}\right),\left(\mathbf{v}^{h}, \Psi^{h}\right)\right) & \\
& +b\left(\left(\mathbf{v}^{h}, \Psi^{h}\right), p^{h}\right) \\
= & F\left(\left(\mathbf{v}^{h}, \Psi^{h}\right)\right) \quad \forall\left(\mathbf{v}^{h}, \Psi^{h}\right) \in \mathscr{W}_{0 n}^{h}(\Omega) .
\end{aligned}
$$

Subtraction of (6.18) from (6.17) yields

$$
\begin{aligned}
& a_{0}\left((\mathbf{u}, \mathbf{B})-\left(\mathbf{u}^{h}, \mathbf{B}^{h}\right),\left(\mathbf{v}^{h}, \Psi^{h}\right)\right)+\tilde{a}_{1}\left((\mathbf{u}, \mathbf{B})-\left(\mathbf{u}^{h}, \mathbf{B}^{h}\right),(\mathbf{u}, \mathbf{B}),\left(\mathbf{v}^{h}, \Psi^{h}\right)\right) \\
& \quad+\tilde{a}_{1}\left(\left(\mathbf{u}^{h}, \mathbf{B}^{h}\right),(\mathbf{u}, \mathbf{B})-\left(\mathbf{u}^{h}, \mathbf{B}^{h}\right),\left(\mathbf{v}^{h}, \Psi^{h}\right)\right)+b\left(\left(\mathbf{v}^{h}, \Psi^{h}\right), p-p^{h}\right) \\
& \quad=0 \quad \forall\left(\mathbf{v}^{h}, \Psi^{h}\right) \in \mathscr{W}_{0 n}^{h}(\Omega) .
\end{aligned}
$$

Then, with $s^{h} \in S_{0}^{h}(\Omega)$ arbitrary, we have

$$
\begin{aligned}
& a_{0}\left(\left(\mathbf{w}^{h}, \boldsymbol{\Phi}^{h}\right)-\left(\mathbf{u}^{h}, \mathbf{B}^{h}\right),\left(\mathbf{v}^{h}, \Psi^{h}\right)\right) \\
& \quad+\tilde{a}_{1}\left(\left(\mathbf{w}^{h}, \boldsymbol{\Phi}^{h}\right)-\left(\mathbf{u}^{h}, \mathbf{B}^{h}\right),(\mathbf{u}, \mathbf{B}),\left(\mathbf{v}^{h}, \Psi^{h}\right)\right) \\
& \quad+\tilde{a}_{1}\left(\left(\mathbf{u}^{h}, \mathbf{B}^{h}\right),\left(\mathbf{w}^{h}, \boldsymbol{\Phi}^{h}\right)-\left(\mathbf{u}^{h}, \mathbf{B}^{h}\right),\left(\mathbf{v}^{h}, \Psi^{h}\right)\right) \\
& \quad+b\left(\left(\mathbf{v}^{h}, \Psi^{h}\right), s^{h}-p^{h}\right) \\
& \quad+a_{0}\left(\left(\mathbf{w}^{h}, \boldsymbol{\Phi}^{h}\right)-(\mathbf{u}, \mathbf{B}),\left(\mathbf{v}^{h}, \Psi^{h}\right)\right) \\
& \quad+\tilde{a}_{1}\left(\left(\mathbf{w}^{h}, \boldsymbol{\Phi}^{h}\right)-(\mathbf{u}, \mathbf{B}),(\mathbf{u}, \mathbf{B}),\left(\mathbf{v}^{h}, \Psi^{h}\right)\right) \\
& \quad+\tilde{a}_{1}\left(\left(\mathbf{u}^{h}, \mathbf{B}^{h}\right),\left(\mathbf{w}^{h}, \boldsymbol{\Phi}^{h}\right)-(\mathbf{u}, \mathbf{B}),\left(\mathbf{v}^{h}, \Psi^{h}\right)\right) \\
& \quad+b\left(\left(\mathbf{v}^{h}, \Psi^{h}\right), s^{h}-p\right) \quad \forall\left(\mathbf{v}^{h}, \Psi^{h}\right) \in \mathscr{W}_{0 n}^{h}(\Omega), \\
& \quad \quad\left(\mathbf{w}^{h}, \boldsymbol{\Phi}^{h}\right) \in \mathscr{W}_{2}^{h}(\Omega), s^{h} \in S_{0}^{h}(\Omega) .
\end{aligned}
$$

Next, set $\left(\mathbf{v}^{h}, \Psi^{h}\right)=\left(\mathbf{w}^{h}, \boldsymbol{\Phi}^{h}\right)-\left(\mathbf{u}^{h}, \mathbf{B}^{h}\right)$ and let $\mathbf{z} \in \mathbf{Z}(\Omega)$ be arbitrary. Then, using (6.3) and the definition of the subspaces $Z(\Omega)$ and $Z^{h}(\Omega)$, we have from (6.19)

$$
\begin{aligned}
a_{0}\left(\left(\mathbf{w}^{h}, \boldsymbol{\Phi}^{h}\right)-\left(\mathbf{u}^{h}, \mathbf{B}^{h}\right),\left(\mathbf{w}^{h}, \boldsymbol{\Phi}^{h}\right)-\left(\mathbf{u}^{h}, \mathbf{B}^{h}\right)\right) \\
\quad+\tilde{a}_{1}\left(\left(\mathbf{w}^{h}, \boldsymbol{\Phi}^{h}\right)-\left(\mathbf{u}^{h}, \mathbf{B}^{h}\right),(\mathbf{u}, \mathbf{B}),\left(\mathbf{w}^{h}, \boldsymbol{\Phi}^{h}\right)-\left(\mathbf{u}^{h}, \mathbf{B}^{h}\right)\right) \\
=a_{0}\left(\left(\mathbf{w}^{h}, \boldsymbol{\Phi}^{h}\right)-(\mathbf{u}, \mathbf{B}),\left(\mathbf{w}^{h}, \boldsymbol{\Phi}^{h}\right)-\left(\mathbf{u}^{h}, \mathbf{B}^{h}\right)\right) \\
\quad+\tilde{a}_{1}\left(\left(\mathbf{w}^{h}, \boldsymbol{\Phi}^{h}\right)-(\mathbf{u}, \mathbf{B}),(\mathbf{u}, \mathbf{B}),\left(\mathbf{w}^{h}, \boldsymbol{\Phi}^{h}\right)-\left(\mathbf{u}^{h}, \mathbf{B}^{h}\right)\right) \\
\quad+\tilde{a}_{1}\left(\left(\mathbf{u}^{h}, \mathbf{B}^{h}\right),\left(\mathbf{w}^{h}, \boldsymbol{\Phi}^{h}\right)-(\mathbf{u}, \mathbf{B}),\left(\mathbf{w}^{h}, \boldsymbol{\Phi}^{h}\right)-\left(\mathbf{u}^{h}, \mathbf{B}^{h}\right)\right) \\
\quad+b\left(\left(\mathbf{w}^{h}, \boldsymbol{\Phi}^{h}\right)-\left(\mathbf{u}^{h}, \mathbf{B}^{h}\right)-(\mathbf{z}, 0), s^{h}-p\right) .
\end{aligned}
$$

The right-hand side of (6.20) may be estimated from above using the continuity properties (4.5)-(4.7). The left-hand side may be estimated from below using 
(4.6) and (4.10). Thus, one easily finds that

$$
\begin{aligned}
{[\min } & \left.\left\{\frac{k_{1}}{M^{2}}, \frac{k_{2}}{R_{m}^{2}}\right\}-\sqrt{2} \gamma_{3} \max \left\{\frac{1}{N}, \frac{\sqrt{2}}{R_{m}}\right\}\|(\mathbf{u}, \mathbf{B})\|_{\mathscr{W}}\right] \\
& \cdot\left\|\left(\mathbf{w}^{h}, \boldsymbol{\Phi}^{h}\right)-\left(\mathbf{u}^{h}, \mathbf{B}^{h}\right)\right\|_{\mathscr{W}} \\
\leq & {\left[\max \left\{\frac{1}{M^{2}}, \frac{5}{R_{m}^{2}}\right\}+\sqrt{2} \gamma_{3} \max \left\{\frac{1}{N}, \frac{\sqrt{2}}{R_{m}}\right\}\right.} \\
\cdot & \left.\cdot\left(\|(\mathbf{u}, \mathbf{B})\|_{\mathscr{W}}+\left\|\left(\mathbf{u}^{h}, \mathbf{B}^{h}\right)\right\|_{\mathscr{W}}\right)\right] \\
& \left.\mathbf{\Phi}^{h}\right)-(\mathbf{u}, \mathbf{B})\left\|_{\mathscr{W}}+\sqrt{3}\left(\frac{\left\|\mathbf{w}^{h}-\mathbf{u}^{h}-\mathbf{z}\right\|_{1}}{\left\|\mathbf{w}^{h}-\mathbf{u}^{h}\right\|_{1}}\right)\right\| s^{h}-p \|_{0} .
\end{aligned}
$$

Now, using the definition of $\Theta$, we have, since $\mathbf{w}^{h}-\mathbf{u}^{h} \in \mathbf{Z}^{h}(\Omega)$,

$$
\inf _{\mathbf{z} \in \mathbf{Z}(\Omega)} \frac{\left\|\mathbf{w}^{h}-\mathbf{u}^{h}-\mathbf{z}\right\|_{1}}{\left\|\mathbf{w}^{h}-\mathbf{u}^{h}\right\|_{1}} \leq \boldsymbol{\theta} .
$$

Furthermore, from (4.25) we have

$$
\alpha_{1}:=\min \left\{\frac{k_{1}}{M^{2}}, \frac{k_{2}}{R_{m}^{2}}\right\}-\sqrt{2} \gamma_{3} \max \left\{\frac{1}{N}, \frac{\sqrt{2}}{R_{m}}\right\}\|(\mathbf{u}, \mathbf{B})\|_{\mathscr{W}}>0 .
$$

Then, taking the infimum of (6.21) over $\mathbf{z} \in \mathbf{Z}(\Omega)$, and using (6.22), (6.23), and the triangle inequality, we obtain

$$
\begin{array}{r}
\left\|(\mathbf{u}, \mathbf{B})-\left(\mathbf{u}^{h}, \mathbf{B}^{h}\right)\right\|_{\mathscr{W}} \\
\leq \frac{\sqrt{3}}{\alpha_{1}} \Theta\left\|p-s^{h}\right\|_{0}+\left(1+\bar{C}_{1}\right)\left\|(\mathbf{u}, \mathbf{B})-\left(\mathbf{w}^{h}, \boldsymbol{\Phi}^{h}\right)\right\|_{\mathscr{W}} \\
\forall s^{h} \in S_{0}^{h}(\Omega),\left(\mathbf{w}^{h}, \boldsymbol{\Phi}^{h}\right) \in \mathscr{W}_{2}^{h}(\Omega),
\end{array}
$$

where

$$
\begin{aligned}
\bar{C}_{1}=\frac{1}{\alpha_{1}}\left[\max \left\{\frac{1}{M^{2}}, \frac{5}{R_{m}^{2}}\right\}+\sqrt{2} \gamma_{3} \max \left\{\frac{1}{N}, \frac{\sqrt{2}}{R_{m}}\right\}\right. \\
\left.\cdot\left(\|(\mathbf{u}, \mathbf{B})\|_{\mathscr{W}}+\left\|\left(\mathbf{u}^{h}, \mathbf{B}^{h}\right)\right\|_{\mathscr{W}}\right)\right] .
\end{aligned}
$$

Now, taking the infimum of (6.24) over $s^{h} \in S_{0}^{h}(\Omega)$ and $\left(\mathbf{w}^{h}, \boldsymbol{\Phi}^{h}\right) \in \mathscr{W}_{2}^{h}(\Omega)$, we have

$$
\begin{aligned}
\left\|(\mathbf{u}, \mathbf{B})-\left(\mathbf{u}^{h}, \mathbf{B}^{h}\right)\right\|_{\mathscr{W}} \leq & \frac{\sqrt{3}}{\alpha_{1}} \Theta \inf _{s^{h} \in S_{0}^{h}(\Omega)}\left\|p-s^{h}\right\|_{0}+\left(1+\bar{C}_{1}\right) \\
& \cdot \inf _{\left(\mathbf{w}^{h}, \boldsymbol{\Phi}^{h}\right) \in \mathscr{W}_{2}^{h}(\Omega)}\left\|(\mathbf{u}, \mathbf{B})-\left(\mathbf{w}^{h}, \boldsymbol{\Phi}^{h}\right)\right\|_{\mathscr{W}} .
\end{aligned}
$$


But, if (6.9) is satisfied, we can show that

$$
\begin{aligned}
& \inf _{\left(\mathbf{w}^{h}, \Phi^{h}\right) \in \mathscr{W}_{2}^{h}(\boldsymbol{\Omega})}\left\|(\mathbf{u}-\mathbf{B})-\left(\mathbf{w}^{h}, \boldsymbol{\Phi}^{h}\right)\right\|_{\mathscr{W}} \\
& \quad \leq\left(1+\frac{\sqrt{3}}{\beta^{h}}\right) \inf _{\left(\mathbf{v}^{h}, \Psi^{h}\right) \in \mathscr{W}_{1}^{h}(\boldsymbol{\Omega})}\left\|(\mathbf{u}, \mathbf{B})-\left(\mathbf{v}^{h}, \Psi^{h}\right)\right\|_{\mathscr{W}} .
\end{aligned}
$$

To show that (6.26) holds, let $\mathbf{v}^{h} \in \mathbf{W}_{1}^{h}(\Omega)$ be arbitrary. It is well known, e.g., see [6] or [7], that (6.9) implies that there exists a unique $\hat{\mathbf{z}}^{h} \in \mathbf{Z}^{h}(\Omega)^{\perp}$ such that

$$
b\left(\left(\hat{\mathbf{z}}^{h}, \mathbf{0}\right), \chi^{h}\right)=b\left(\left(\mathbf{u}-\mathbf{v}^{h}, \mathbf{0}\right), \chi^{h}\right) \quad \forall \chi^{h} \in S_{0}^{h}(\mathbf{\Omega})
$$

and

$$
\left\|\hat{\mathbf{z}}^{h}\right\|_{1} \leq \frac{\sqrt{3}}{\beta^{h}}\left\|\mathbf{u}-\mathbf{v}^{h}\right\|_{1} .
$$

Now, let $\mathbf{w}^{h}=\hat{\mathbf{z}}^{h}+\mathbf{v}^{h}$. We then have that $\mathbf{w}^{h} \in \mathbf{X}^{h}(\Omega)$ satisfies $\left.\mathbf{w}^{h}\right|_{\partial \Omega}=\left.\mathbf{v}^{h}\right|_{\partial \Omega}=$ $\mathbf{g}^{h}$ and

$$
\begin{aligned}
b\left(\left(\mathbf{w}^{h}, \boldsymbol{\Phi}^{h}\right), \chi^{h}\right) & =b\left(\left(\mathbf{w}^{h}, \mathbf{0}\right), \chi^{h}\right)=b\left(\left(\hat{\mathbf{z}}^{h}+\mathbf{v}^{h}, \mathbf{0}\right), \chi^{h}\right) \\
& =b\left((\mathbf{u}, \mathbf{0}), \chi^{h}\right)=0 \quad \forall \chi^{h} \in S_{0}^{h}(\Omega),
\end{aligned}
$$

so that $\mathbf{w}^{h} \in \mathbf{w}_{2}^{h}(\Omega)$. Moreover,

$$
\left\|\mathbf{u}-\mathbf{w}^{h}\right\|_{1} \leq\left\|\mathbf{u}-\mathbf{v}^{h}\right\|_{1}+\left\|\hat{\mathbf{z}}^{h}\right\|_{1} \leq\left(1+\frac{\sqrt{3}}{\beta^{h}}\right)\left\|\mathbf{u}-\mathbf{v}^{h}\right\|_{1} .
$$

Since $\mathbf{v}^{h}$ is arbitrary, it follows that

$$
\inf _{\mathbf{w}^{h} \in \mathbf{W}_{2}^{h}(\Omega)}\left\|\mathbf{u}-\mathbf{w}^{h}\right\|_{1} \leq\left(1+\frac{\sqrt{3}}{\beta^{h}}\right) \inf _{\mathbf{v}^{h} \in \mathbf{W}_{1}^{h}(\Omega)}\left\|\mathbf{u}-\mathbf{v}^{h}\right\|_{1} .
$$

Then,

$$
\begin{aligned}
& \inf _{\left(\mathbf{w}^{h}, \boldsymbol{\Phi}^{h}\right) \in \mathscr{W}_{2}^{h}(\Omega)}\left\|(\mathbf{u}, \mathbf{B})-\left(\mathbf{w}^{h}, \boldsymbol{\Phi}^{h}\right)\right\|_{\mathscr{W}}^{2} \\
& \quad=\inf _{\mathbf{w}^{h} \in \mathbf{W}_{2}^{h}(\Omega)}\left\|\mathbf{u}-\mathbf{w}^{h}\right\|_{1}^{2}+\inf _{\boldsymbol{\Phi}^{h} \in \mathbf{Y}_{1}^{h}(\Omega)}\left\|\mathbf{B}-\boldsymbol{\Phi}^{h}\right\|_{1}^{2} \\
& \leq\left(1+\frac{\sqrt{3}}{\beta^{h}}\right)^{2}\left[\inf _{\mathbf{v}^{h} \in \mathbf{W}_{1}^{h}(\Omega)}\left\|\mathbf{u}-\mathbf{v}^{h}\right\|_{1}^{2}+\inf _{\Psi^{h} \in \mathbf{Y}_{1}^{h}(\Omega)}\left\|\mathbf{B}-\Psi^{h}\right\|_{1}^{2}\right] \\
& \quad=\left(1+\frac{\sqrt{3}}{\beta^{h}}\right)^{2} \inf _{\left(\mathbf{v}^{h}, \Psi^{h}\right) \in \mathscr{W}_{1}^{h}(\Omega)}\left\|(\mathbf{u}, \mathbf{B})-\left(\mathbf{v}^{h}, \Psi^{h}\right)\right\|_{\mathscr{W}}^{2},
\end{aligned}
$$

so that $(6.26)$ follows.

Substitution of (6.26) into (6.25) then yields (6.15) with

$$
C_{1}=\left(1+\bar{C}_{1}\right)\left(1+\sqrt{3} / \beta^{h}\right) \text { and } C_{2}=\sqrt{3} / \alpha_{1} .
$$


For an arbitrary $s^{h} \in S_{0}^{h}(\Omega)$, we have from (6.17) and (6.18),

$$
\begin{array}{r}
b\left(\left(\mathbf{v}^{h}, \Psi^{h}\right), s^{h}-p^{h}\right)=b\left(\left(\mathbf{v}^{h}, \Psi^{h}\right), s^{h}-p\right)-a_{0}\left((\mathbf{u}, \mathbf{B})-\left(\mathbf{u}^{h}, \mathbf{B}^{h}\right),\left(\mathbf{v}^{h}, \Psi^{h}\right)\right) \\
-\tilde{a}_{1}\left((\mathbf{u}, \mathbf{B})-\left(\mathbf{u}^{h}, \mathbf{B}^{h}\right),(\mathbf{u}, \mathbf{B}),\left(\mathbf{v}^{h}, \Psi^{h}\right)\right) \\
-\tilde{a}_{1}\left(\left(\mathbf{u}^{h}, \mathbf{B}^{h}\right),(\mathbf{u}, \mathbf{B})-\left(\mathbf{u}^{h}, \mathbf{B}^{h}\right),\left(\mathbf{v}^{h}, \Psi^{h}\right)\right) \\
\forall\left(\mathbf{v}^{h}, \Psi^{h}\right) \in \mathscr{W}_{0 n}^{h}(\mathbf{\Omega}) .
\end{array}
$$

Using the continuity of $a_{0}(\cdot, \cdot)$ and $\tilde{a}_{1}(\cdot, \cdot, \cdot)$ and the inf-sup condition (6.9) then yields

$$
\begin{array}{r}
\left\|s^{h}-p^{h}\right\|_{0} \leq \frac{1}{\beta^{h}}\left[\sqrt{3}\left\|s^{h}-p\right\|_{0}+\left(\max \left\{\frac{1}{M^{2}}, \frac{5}{R_{m}^{2}}\right\}+\sqrt{2} \gamma_{3} \max \left\{\frac{1}{N}, \frac{\sqrt{2}}{R_{m}}\right\}\right.\right. \\
\left.\left.\cdot\left[\|(\mathbf{u}, \mathbf{B})\|_{\mathscr{W}}+\left\|\left(\mathbf{u}^{h}, \mathbf{B}^{h}\right)\right\|_{\mathscr{W}}\right]\right)\left\|(\mathbf{u}-\mathbf{B})-\left(\mathbf{u}^{h}, \mathbf{B}^{h}\right)\right\|_{\mathscr{W}}\right] .
\end{array}
$$

Then, using the triangle inequality, (6.15), and by taking appropriate infima, we are easily led to (6.16) with $C_{3}=\bar{C}_{3} C_{1} / \beta^{h}$ and $C_{4}=1+\left(\bar{C}_{3} C_{2} \Theta / \beta^{h}\right)+\sqrt{3} / \beta^{h}$, where

$$
\bar{C}_{3}=\max \left\{\frac{1}{M^{2}}, \frac{5}{R_{m}^{2}}\right\}+\sqrt{2} \gamma_{3} \max \left\{\frac{1}{N}, \frac{\sqrt{2}}{R_{m}}\right\}\left(\|(\mathbf{u}, \mathbf{B})\|_{\mathscr{W}}+\left\|\left(\mathbf{u}^{h}, \mathbf{B}^{h}\right)\right\|_{\mathscr{W}}\right) .
$$

One may obtain an upper bound for $\bar{C}_{1}$ and $\bar{C}_{3}$ through the use of (4.22) and the corresponding bound for $\left(\mathbf{u}^{h}, \mathbf{B}^{h}\right)$.

We remark that the error estimates (6.15) and (6.16) are optimal with respect to the graph norms employed. The right-hand side of these estimates involve approximation-theoretic terms using spaces constrained to satisfy boundary conditions. For a discussion of the approximation theory in such spaces, see [5]. Of course, similar estimates can be derived in the context of the alternative boundary conditions (2.8), (2.11), and (2.12).

Theorem 6.4 provides an estimate for the velocity and magnetic field error measured in the $\mathbf{H}^{1}(\Omega)$-norm. We now turn to obtaining estimates for these errors in the $\mathbf{L}^{2}(\Omega)$-norm. Such estimates are obtained using standard "duality arguments" with substantial modifications to account for the inhomogeneous essential boundary conditions. For the use of such procedures for the NavierStokes equations with homogeneous boundary conditions, one may consult [6] or [7]; for the inhomogeneous case, see [5].

The key ingredient in obtaining $\mathbf{L}^{2}(\Omega)$-norm error estimates is to introduce the following dual problem. We seek $(\mathbf{w}, \boldsymbol{\Phi}) \in \mathscr{W}_{0 n}(\Omega)$ and $s \in L_{0}^{2}(\Omega)$ such that

$$
\begin{aligned}
a_{0}((\mathbf{v}, \Psi),(\mathbf{w}, \boldsymbol{\Phi}))+\tilde{a}_{1}((\mathbf{u}, \mathbf{B}),(\mathbf{v}, \Psi),(\mathbf{w}, \boldsymbol{\Phi})) \\
\quad+\tilde{a}_{1}((\mathbf{v}, \boldsymbol{\Psi}),(\mathbf{u}, \mathbf{B}),(\mathbf{w}, \boldsymbol{\Phi}))+b((\mathbf{v}, \Psi), s) \\
=\left((\mathbf{u}, \mathbf{B})-\left(\mathbf{u}^{h}, \mathbf{B}^{h}\right),(\mathbf{v}, \boldsymbol{\Psi})\right) \quad \forall(\mathbf{v}, \Psi) \in \mathscr{W}_{0 n}(\Omega)
\end{aligned}
$$


and

$$
b((\mathbf{w}, \boldsymbol{\Phi}), \psi)=0 \quad \forall \psi \in L_{0}^{2}(\Omega),
$$

where $(\cdot, \cdot)$ denotes the inner product in $\mathbf{L}^{2}(\Omega)$. The left-hand side of (6.27)(6.28) is merely the adjoint of the operator on the left-hand side of (3.7)-(3.8) with $a_{1}(\cdot, \cdot, \cdot)$ replaced by $\tilde{a}_{1}(\cdot, \cdot, \cdot)$, linearized about $(\mathbf{u}, \mathbf{B})$. If we assume the hypotheses of Theorem 4.7 , then one may easily deduce that $(6.27)-(6.28)$ has a unique solution.

We assume that the solution of (6.27)-(6.28) satisfies $\mathbf{w} \in \mathbf{H}^{2}(\Omega), \Phi \in$ $\mathbf{H}^{2}(\Omega)$, and $s \in H^{1}(\Omega)$, and moreover, that there exists a constant $C>0$ such that

$$
\|(\mathbf{W}, \boldsymbol{\Phi})\|_{\mathbf{H}^{2} \times \mathbf{H}^{2}}+\|s\|_{H^{1}} \leq C\left\|(\mathbf{u}, \mathbf{B})-\left(\mathbf{u}^{h}, \mathbf{B}^{h}\right)\right\|_{\mathbf{L}^{2} \times \mathbf{L}^{2}} \text {. }
$$

This additional regularity and a priori estimate require smoothness assumptions about $(\mathbf{u}, \mathbf{B})$ and the domain $\Omega$. On convex polyhedral domains, this additional regularity for $(\mathbf{u}, \mathbf{B})$ has not been shown; indeed, even for the uncoupled Navier-Stokes equations with inhomogeneous velocity boundary conditions, it is not known, under general conditions, that velocity solutions on convex polyhedral domains belong to $\mathbf{H}^{2}(\Omega)$. Furthermore, even if this additional regularity of $(\mathbf{u}, \mathbf{B})$ were known, we would still be faced, on convex polyhedral domains, with the unsolved problem of the regularity of solutions of the linear systems such as (6.27)-(6.28) with corresponding nonstandard boundary conditions such as homogeneous versions of (2.21)-(2.23). However, it seems reasonable to assume, under certain conditions, that (6.29) is valid, even for convex polyhedral domains.

We can now derive our $\mathbf{L}^{2}(\Omega)$-norm estimates.

Theorem 6.5. Let the hypothesis of Theorem 6.4 hold. Let $((\mathbf{w}, \boldsymbol{\Phi}), s)$ denote the solution of (6.27)-(6.28), and assume that (6.29) holds. Then there exists a constant $C>0$, independent of $h$, such that

$$
\left\|(\mathbf{u}, \mathbf{B})-\left(\mathbf{u}^{h}, \mathbf{B}^{h}\right)\right\|_{\mathbf{L}^{2} \times \mathbf{L}^{2}}
$$

Proof. Subtraction of (6.18) from (6.17) yields

$$
\begin{aligned}
a_{0}\left((\mathbf{u}, \mathbf{B})-\left(\mathbf{u}^{h}, \mathbf{B}^{h}\right),\left(\mathbf{v}^{h}, \Psi^{h}\right)\right) \\
\quad+\tilde{a}_{1}\left((\mathbf{u}, \mathbf{B})-\left(\mathbf{u}^{h}, \mathbf{B}^{h}\right),(\mathbf{u}, \mathbf{B}),\left(\mathbf{v}^{h}, \Psi^{h}\right)\right) \\
\quad+\tilde{a}_{1}\left((\mathbf{u}, \mathbf{B}),(\mathbf{u}, \mathbf{B})-\left(\mathbf{u}^{h}, \mathbf{B}^{h}\right),\left(\mathbf{v}^{h}, \Psi^{h}\right)\right) \\
\quad-\tilde{a}_{1}\left((\mathbf{u}, \mathbf{B})-\left(\mathbf{u}^{h}, \mathbf{B}^{h}\right),(\mathbf{u}, \mathbf{B})-\left(\mathbf{u}^{h}, \mathbf{B}^{h}\right),\left(\mathbf{v}^{h}, \Psi^{h}\right)\right) \\
\quad+b\left(\left(\mathbf{v}^{h}, \Psi^{h}\right), p-p^{h}\right)=0 \quad \forall\left(\mathbf{v}^{h}, \Psi^{h}\right) \in \mathscr{W}_{0 n}^{h}(\Omega) .
\end{aligned}
$$


Subtraction of (6.7) from (3.8) yields

$$
b\left((\mathbf{u}, \mathbf{B})-\left(\mathbf{u}^{h}, \mathbf{B}^{h}\right), \phi^{h}\right)=0 \quad \forall \phi^{h} \in S_{0}^{h}(\Omega) .
$$

Recall the splitting $\mathbf{u}=\hat{\mathbf{u}}+\mathbf{u}_{0}, \mathbf{B}=\widehat{\mathbf{B}}+\mathbf{B}_{0}, \mathbf{u}^{h}=\hat{\mathbf{u}}^{h}+\mathbf{u}_{0}^{h}$, and $\mathbf{B}^{h}=\widehat{\mathbf{B}}^{h}+\mathbf{B}_{0}^{h}$.

Now in (6.27), set

$$
(\mathbf{v}, \boldsymbol{\Psi})=(\mathbf{u}, \mathbf{B})-\left(\mathbf{u}^{h}, \mathbf{B}^{h}\right)-\left(\mathbf{u}_{0}, \mathbf{B}_{0}\right)+\left(\mathbf{u}_{0}^{h}, \mathbf{B}_{0}^{h}\right) \in \mathscr{W}_{0 n},
$$

and in (6.28) set

$$
\psi=p-p^{h} \in L_{0}^{2}(\Omega) .
$$

Combining the result with (6.31) and (6.32) yields

$$
\begin{aligned}
\|(\mathbf{u}, \mathbf{B}) & -\left(\mathbf{u}^{h}, \mathbf{B}^{h}\right) \|_{\mathbf{L}^{2} \times \mathbf{L}^{2}}^{2} \\
= & a_{0}\left((\mathbf{u}, \mathbf{B})-\left(\mathbf{u}^{h}, \mathbf{B}^{h}\right),(\mathbf{w}, \mathbf{\Phi})-\left(\mathbf{v}^{h}, \Psi^{h}\right)\right) \\
& +\tilde{a}_{1}\left((\mathbf{u}, \mathbf{B})-\left(\mathbf{u}^{h}, \mathbf{B}^{h}\right),(\mathbf{u}, \mathbf{B}),(\mathbf{w}, \boldsymbol{\Phi})-\left(\mathbf{v}^{h}, \Psi^{h}\right)\right) \\
& +\tilde{a}_{1}\left((\mathbf{u}, \mathbf{B}),(\mathbf{u}, \mathbf{B})-\left(\mathbf{u}^{h}, \mathbf{B}^{h}\right),(\mathbf{w}, \boldsymbol{\Phi})-\left(\mathbf{v}^{h}, \Psi^{h}\right)\right) \\
& +\tilde{a}_{1}\left((\mathbf{u}, \mathbf{B})-\left(\mathbf{u}^{h}, \mathbf{B}^{h}\right),(\mathbf{u}, \mathbf{B})-\left(\mathbf{u}^{h}, \mathbf{B}^{h}\right),\left(\mathbf{v}^{h}, \Psi^{h}\right)\right) \\
& +b\left((\mathbf{u}, \mathbf{B})-\left(\mathbf{u}^{h}, \mathbf{B}^{h}\right), s-\phi^{h}\right) \\
& +b\left((\mathbf{w}, \boldsymbol{\Phi})-\left(\mathbf{v}^{h}, \Psi^{h}\right), p-p^{h}\right) \\
& +\left(\left(\mathbf{u}_{0}, \mathbf{B}\right)_{0}-\left(\mathbf{u}_{0}^{h}, \mathbf{B}_{0}^{h}\right),(\mathbf{u}, \mathbf{B})-\left(\mathbf{u}^{h}, \mathbf{B}^{h}\right)\right) \\
& -a_{0}\left(\left(\mathbf{u}_{0}, \mathbf{B}_{0}\right)-\left(\mathbf{u}_{0}^{h}, \mathbf{B}_{0}^{h}\right),(\mathbf{w}, \boldsymbol{\Phi})\right) \\
& -\tilde{a}_{1}\left(\left(\mathbf{u}_{0}, \mathbf{B}_{0}\right)-\left(\mathbf{u}_{0}^{h}, \mathbf{B}_{0}^{h}\right),(\mathbf{u}, \mathbf{B}),(\mathbf{w}, \boldsymbol{\Phi})\right) \\
& -\tilde{a}_{1}\left((\mathbf{u}, \mathbf{B}),\left(\mathbf{u}_{0}, \mathbf{B}_{0}\right)-\left(\mathbf{u}_{0}^{h}, \mathbf{B}_{0}^{h}\right),(\mathbf{w}, \boldsymbol{\Phi})\right) \\
& -b\left(\left(\mathbf{u}_{0}, \mathbf{B}_{0}\right)-\left(\mathbf{u}_{0}^{h}, \mathbf{B}_{0}^{h}\right), s\right) \quad \forall\left(\mathbf{v}^{h}, \Psi^{h}\right) \in \mathscr{W}_{0 n}^{h}(\Omega), \phi^{h} \in S_{0}^{h}(\boldsymbol{\Omega}) .
\end{aligned}
$$

One easily sees, by integrating by parts, that $(6.27)-(6.28)$ imply that

$$
\begin{gathered}
-\frac{1}{M^{2}} \Delta \mathbf{w}+\frac{1}{N}\left[\mathbf{w} \cdot(\nabla \mathbf{u})^{T}-\mathbf{u} \cdot \nabla \mathbf{w}\right]+\frac{1}{R_{m}}(\nabla \times \boldsymbol{\Phi}) \times \mathbf{B}+\nabla s=\mathbf{u}-\mathbf{u}^{h} \text { in } \Omega, \\
\frac{1}{R_{m}^{2}}[\nabla \times(\nabla \times \boldsymbol{\Phi})-\nabla(\nabla \cdot \boldsymbol{\Phi})] \\
+\frac{1}{R_{m}}[(\nabla \times \mathbf{B}) \times \mathbf{w}-(\nabla \times \boldsymbol{\Phi}) \times \mathbf{u}-\nabla \times(\mathbf{B} \times \mathbf{w})]=\mathbf{B}-\mathbf{B}^{h} \text { in } \Omega, \\
\quad \nabla \cdot \mathbf{w}=0 \text { in } \Omega, \\
\left.\mathbf{w}\right|_{\partial \Omega}=0,\left.\quad(\mathbf{B} \cdot \mathbf{n})\right|_{\partial \Omega}=0,\left.\quad\left(\frac{1}{R_{m}}(\nabla \times \mathbf{\Phi}) \times \mathbf{n}+\mathbf{w} \times \mathbf{B} \times \mathbf{n}\right)\right|_{\partial \Omega}=0 \text { on } \partial \Omega,
\end{gathered}
$$


where these equations hold in $\mathbf{H}^{-1}(\Omega), \mathbf{H}^{-1}(\Omega), L^{2}(\Omega), \mathbf{H}^{1 / 2}(\partial \Omega), H^{1 / 2}(\partial \Omega)$, and $\mathbf{H}^{-1 / 2}(\partial \Omega)$, respectively. On the other hand, since $(\mathbf{w}, \Phi) \in \mathbf{H}^{2}(\Omega) \times$ $\mathbf{H}^{2}(\Omega)$ and $s \in H^{1}(\Omega)$, all the terms in the first two equations are in $\mathbf{L}^{2}(\Omega)$, so that these equations hold in $\mathbf{L}^{2}(\Omega)$ as well. Likewise, the last four equations hold in $H^{1}(\Omega), \mathbf{H}^{3 / 2}(\partial \Omega), H^{3 / 2}(\partial \Omega)$, and $\mathbf{H}^{1 / 2}(\partial \Omega)$. Then, multiplying the first equation by $\left(\mathbf{u}_{0}-\mathbf{u}_{0}^{h}\right)$ and the second by $\left(\mathbf{B}_{0}-\mathbf{B}_{0}^{h}\right)$, adding the results, integrating by parts, and using the boundary conditions on $\mathbf{w}$ and $\Phi$, we obtain

$$
\begin{aligned}
& \left(\left(\mathbf{u}_{0}, \mathbf{B}_{0}\right)-\left(\mathbf{u}_{0}^{h}, \mathbf{B}_{0}^{h}\right),(\mathbf{u}, \mathbf{B})-\left(\mathbf{u}^{h}, \mathbf{B}^{h}\right)\right) \\
& \quad-a_{0}\left(\left(\mathbf{u}_{0}, \mathbf{B}_{0}\right)-\left(\mathbf{u}_{0}^{h}, \mathbf{B}_{0}^{h}\right),(\mathbf{w}, \boldsymbol{\Phi})\right) \\
& \quad-\tilde{a}_{1}\left(\left(\mathbf{u}_{0}, \mathbf{B}_{0}\right)-\left(\mathbf{u}_{0}^{h}, \mathbf{B}_{0}^{h}\right),(\mathbf{u}, \mathbf{B}),(\mathbf{w}, \mathbf{\Phi})\right) \\
& \quad-\tilde{a}_{1}\left((\mathbf{u}, \mathbf{B}),\left(\mathbf{u}_{0}, \mathbf{B}_{0}\right)-\left(\mathbf{u}_{0}^{h}, \mathbf{B}_{0}^{h}\right),(\mathbf{w}, \boldsymbol{\Phi})\right) \\
& \quad-b\left(\left(\mathbf{u}_{0}, \mathbf{B}_{0}\right)-\left(\mathbf{u}_{0}^{h}, \mathbf{B}_{0}^{h}\right), s\right) \\
& =\int_{\partial \Omega}\left\{-\frac{1}{M^{2}} \mathbf{n} \cdot \nabla \mathbf{w} \cdot\left(\mathbf{u}_{0}-\mathbf{u}_{0}^{h}\right)+s\left(\mathbf{u}_{0}-\mathbf{u}_{0}^{h}\right) \cdot \mathbf{n}\right. \\
& \left.\quad-\frac{1}{R_{m}^{2}} \nabla \cdot \boldsymbol{\Phi}\left(\mathbf{B}_{0}-\mathbf{B}_{0}^{h}\right) \cdot \mathbf{n}\right\} d \mathbf{x} .
\end{aligned}
$$

Recall that $\left.\mathbf{u}_{0}\right|_{\partial \Omega}=\mathbf{g},\left.\left(\mathbf{B}_{0} \cdot \mathbf{n}\right)\right|_{\partial \Omega}=q,\left.\mathbf{u}_{0}^{h}\right|_{\partial \Omega}=\mathbf{g}^{h}$, and $\left.\left(\mathbf{B}_{0}^{h} \cdot \mathbf{n}\right)\right|_{\partial \Omega}=q^{h}$. Substituting these into (6.34) and then the latter into (6.33), we obtain

$$
\begin{aligned}
\|(\mathbf{u}, \mathbf{B}) & -\left(\mathbf{u}^{h}, \mathbf{B}^{h}\right) \|_{\mathbf{L}^{2} \times \mathbf{L}^{2}}^{2} \\
= & a_{0}\left((\mathbf{u}, \mathbf{B})-\left(\mathbf{u}^{h}, \mathbf{B}^{h}\right),(\mathbf{w}, \mathbf{\Phi})-\left(\mathbf{v}^{h}, \Psi^{h}\right)\right) \\
& +\tilde{a}_{1}\left((\mathbf{u}, \mathbf{B})-\left(\mathbf{u}^{h}, \mathbf{B}^{h}\right),(\mathbf{u}, \mathbf{B}),(\mathbf{w}, \boldsymbol{\Phi})-\left(\mathbf{v}^{h}, \Psi^{h}\right)\right) \\
& +\tilde{a}_{1}\left((\mathbf{u}, \mathbf{B}),(\mathbf{u}, \mathbf{B})-\left(\mathbf{u}^{h}, \mathbf{B}^{h}\right),(\mathbf{w}, \boldsymbol{\Phi})-\left(\mathbf{v}^{h}, \Psi^{h}\right)\right) \\
& +b\left((\mathbf{u}, \mathbf{B})-\left(\mathbf{u}^{h}, \mathbf{B}^{h}\right), s-\phi^{h}\right)+b\left((\mathbf{w}, \mathbf{\Phi})-\left(\mathbf{v}^{h}, \Psi^{h}\right), p-p^{h}\right) \\
& -\tilde{a}_{1}\left((\mathbf{u}, \mathbf{B})-\left(\mathbf{u}^{h}, \mathbf{B}^{h}\right),(\mathbf{u}, \mathbf{B})-\left(\mathbf{u}^{h}, \mathbf{B}^{h}\right),(\mathbf{w}, \boldsymbol{\Phi})-\left(\mathbf{v}^{h}, \Psi^{h}\right)\right) \\
& +\tilde{a}_{1}\left((\mathbf{u}, \mathbf{B})-\left(\mathbf{u}^{h}, \mathbf{B}^{h}\right),(\mathbf{u}, \mathbf{B})-\left(\mathbf{u}^{h}, \mathbf{B}^{h}\right),(\mathbf{w}, \boldsymbol{\Phi})\right) \\
& +\int_{\partial \Omega}\left\{-\frac{1}{M^{2}} \mathbf{n} \cdot \nabla \mathbf{w} \cdot\left(\mathbf{g}-\mathbf{g}^{h}\right)+s\left(\mathbf{g}-\mathbf{g}^{h}\right) \cdot \mathbf{n}-\frac{1}{R_{m}^{2}}(\nabla \cdot \boldsymbol{\Phi})\left(q-q^{h}\right)\right\} d \mathbf{x} \\
& \forall\left(\mathbf{v}^{h}, \Psi^{h}\right) \in \mathscr{W}_{0 n}^{h}(\Omega), \phi^{h} \in S_{0}^{h}(\Omega) .
\end{aligned}
$$

Using the various continuity properties of the forms (see $\S 4.2$ ), we then have 
that

$$
\begin{aligned}
\|(\mathbf{u}, \mathbf{B})- & \left(\mathbf{u}^{h}, \mathbf{B}^{h}\right) \|_{\mathbf{L}^{2} \times \mathbf{L}^{2}}^{2} \\
\leq & {\left[\max \left\{\frac{1}{M^{2}}, \frac{5}{R_{m}^{2}}\right\}\left\|(\mathbf{u}, \mathbf{B})-\left(\mathbf{u}^{h}, \mathbf{B}^{h}\right)\right\|_{\mathscr{W}}\right.} \\
& +\sqrt{2} \max \left\{\frac{\gamma_{3}}{N}, \frac{\sqrt{2} \gamma_{3}}{R_{m}}\right\}\left(2\|(\mathbf{u}, \mathbf{B})\|_{\mathscr{W}}+\left\|(\mathbf{u}, \mathbf{B})-\left(\mathbf{u}^{h}, \mathbf{B}^{h}\right)\right\|_{\mathscr{W}}\right) \\
& \left.\cdot\left\|(\mathbf{u}, \mathbf{B})-\left(\mathbf{u}^{h}, \mathbf{B}^{h}\right)\right\|_{\mathscr{W}}+\sqrt{3}\left\|p-p^{h}\right\|_{0}\right]\left\|(\mathbf{w}, \mathbf{\Phi})-\left(\mathbf{v}^{h}, \Psi^{h}\right)\right\|_{\mathscr{W}} \\
+ & \sqrt{2} \max \left\{\frac{\gamma_{3}}{N}, \frac{\sqrt{2} \gamma_{3}}{R_{m}}\right\}\left\|(\mathbf{u}, \mathbf{B})-\left(\mathbf{u}^{h}, \mathbf{B}^{h}\right)\right\|_{\mathscr{W}}^{2}\|(\mathbf{w}, \mathbf{\Phi})\|_{\mathscr{W}} \\
+ & \sqrt{3}\left\|(\mathbf{u}, \mathbf{B})-\left(\mathbf{u}^{h}, \mathbf{B}^{h}\right)\right\|_{\mathscr{W}}\left\|s-\phi^{h}\right\|_{0} \\
+ & \frac{1}{M^{2}}\|\nabla \mathbf{w} \cdot \mathbf{n}\|_{1 / 2, \partial \Omega}\left\|\mathbf{g}-\mathbf{g}^{h}\right\|_{-1 / 2, \partial \Omega} \\
+ & \|s\|_{1 / 2, \partial \Omega}\left\|\mathbf{g} \cdot \mathbf{n}-\mathbf{g}^{h} \cdot \mathbf{n}\right\|_{-1 / 2, \partial \Omega} \\
+ & \frac{1}{R_{m}}\|\nabla \cdot \mathbf{\Phi}\|_{1 / 2, \partial \Omega}\left\|q-q^{h}\right\|_{-1 / 2, \partial \Omega}
\end{aligned}
$$

Now, from approximation theory and (6.29), we have that

$$
\begin{gathered}
\inf _{\left(\mathbf{v}^{h}, \Psi^{h}\right) \in \mathscr{W}_{0 n}^{h}(\Omega)}\left\|(\mathbf{w}, \boldsymbol{\Phi})-\left(\mathbf{v}^{h}, \mathbf{\Psi}^{h}\right)\right\|_{\mathscr{W}} \leq C h\|(\mathbf{w}, \boldsymbol{\Phi})\|_{\mathbf{H}^{2} \times \mathbf{H}^{2}} \\
\quad \leq C h\left\|(\mathbf{u}, \mathbf{B})-\left(\mathbf{u}^{h}, \mathbf{B}^{h}\right)\right\|_{\mathbf{L}^{2} \times \mathbf{L}^{2}} \\
\inf _{\phi^{h} \in S_{0}^{h}(\Omega)}\left\|s-\phi^{h}\right\|_{0} \leq C h\|s\|_{1} \leq C h\left\|(\mathbf{u}, \mathbf{B})-\left(\mathbf{u}^{h}, \mathbf{B}^{h}\right)\right\|_{\mathbf{L}^{2} \times \mathbf{L}^{2}}, \\
\|(\mathbf{w}, \boldsymbol{\Phi})\|_{\mathscr{W}} \leq\|(\mathbf{w}, \boldsymbol{\Phi})\|_{\mathbf{H}^{2} \times \mathbf{H}^{2}} \leq C\left\|(\mathbf{u}, \mathbf{B})-\left(\mathbf{u}^{h}, \mathbf{B}^{h}\right)\right\|_{\mathbf{L}^{2} \times \mathbf{L}^{2}}, \\
\|\nabla \mathbf{w} \cdot \mathbf{n}\|_{1 / 2, \partial \Omega} \leq C\|\mathbf{w}\|_{2} \leq C\left\|(\mathbf{u}, \mathbf{B})-\left(\mathbf{u}^{h}, \mathbf{B}^{h}\right)\right\|_{\mathbf{L}^{2} \times \mathbf{L}^{2}}, \\
\|s\|_{1 / 2, \partial \Omega} \leq C\|s\|_{1} \leq C\left\|(\mathbf{u}, \mathbf{B})-\left(\mathbf{u}^{h}, \mathbf{B}^{h}\right)\right\|_{\mathbf{L}^{2} \times \mathbf{L}^{2}}, \\
\|\nabla \cdot \mathbf{w}\|_{1 / 2, \partial \Omega} \leq C\|\mathbf{w}\|_{2} \leq C\left\|(\mathbf{u}, \mathbf{B})-\left(\mathbf{u}^{h}, \mathbf{B}^{h}\right)\right\|_{\mathbf{L}^{2} \times \mathbf{L}^{2}}
\end{gathered}
$$

Combining these inequalities with (6.35) then yields (6.30).

Whether or not the estimate (6.30) provides an improvement over the estimate (6.15) depends on the last three terms on the right-hand side of (6.30). For a discussion of estimates for $\left\|\mathbf{g}-\mathbf{g}^{h}\right\|_{1 / 2, \partial \Omega}$ and $\left\|q-q^{h}\right\|_{-1 / 2, \partial \Omega}$, see [5]; 
for the last term, one also has to consider the smoothness of $\mathbf{n}$. In any case, the last three terms in (6.30) are at least $O\left(h^{1 / 2}\right)$ and in many situations are $O(h)$. Thus, estimate (6.30) provides at least an $O\left(h^{1 / 2}\right)$, and perhaps an $O(h)$ improvement over the estimate (6.15).

\section{SOLUTION METHODS}

We describe three possible iterative schemes for solving the nonlinear algebraic equations which result from discretization. We describe the methods in the context of the continuous problem; the results hold as well for the discrete problems.

In the first scheme we find, at each iteration,

$$
\begin{gathered}
(\mathbf{u}, \mathbf{B})_{m+1} \in\left\{\mathbf{w} \in \mathbf{H}^{1}(\Omega):\left.\mathbf{w}\right|_{\partial \Omega}=\mathbf{g}\right\} \times\left\{\boldsymbol{\Phi} \in \mathbf{H}^{1}(\Omega):\left.(\boldsymbol{\Phi} \cdot \mathbf{n})\right|_{\partial \Omega}=q\right\} \\
\text { and } p_{m+1} \in L_{0}^{2}(\Omega)
\end{gathered}
$$

such that

$$
\begin{gathered}
\tilde{a}\left((\mathbf{u}, \mathbf{B})_{m},(\mathbf{u}, \mathbf{B})_{m+1},(\mathbf{v}, \Psi)\right)+b\left((\mathbf{v}, \Psi), p_{m+1}\right) \\
=F((\mathbf{v}, \Psi)) \quad \forall(\mathbf{v}, \Psi) \in \mathscr{W}_{0 n}(\Omega)
\end{gathered}
$$

and

$$
b\left((\mathbf{u}, \mathbf{B})_{m+1}, \chi\right)=0 \quad \forall \chi \in L_{0}^{2}(\Omega) .
$$

This iteration scheme has the advantage that it converges for any initial guess whenever one can guarantee the uniqueness of the solutions; its disadvantage is that it results in a large coupled system of linear equations.

In the second iteration scheme we find, at each iteration,

$$
\begin{gathered}
(\mathbf{u}, \mathbf{B})_{m+1} \in\left\{\mathbf{w} \in \mathbf{H}^{1}(\Omega):\left.\mathbf{w}\right|_{\partial \Omega}=\mathbf{g}\right\} \times\left\{\boldsymbol{\Phi} \in \mathbf{H}^{1}(\Omega):\left.(\boldsymbol{\Phi} \cdot \mathbf{n})\right|_{\partial \Omega}=q\right\} \\
\text { and } p_{m+1} \in L_{0}^{2}(\Omega)
\end{gathered}
$$

such that

$$
\begin{aligned}
& a_{0}\left((\mathbf{u}, \mathbf{B})_{m+1},(\mathbf{v}, \Psi)\right)+b\left((\mathbf{v}, \Psi), p_{m+1}\right) \\
& \quad=F((\mathbf{v}, \Psi))-\tilde{a}_{1}\left((\mathbf{u}, \mathbf{B})_{m},(\mathbf{u}, \mathbf{B})_{m},(\mathbf{v}, \Psi)\right) \quad \forall(\mathbf{v}, \Psi) \in \mathscr{W}_{0 n}(\Omega)
\end{aligned}
$$

and

$$
b\left((\mathbf{u}, \mathbf{B})_{m+1}, \chi\right)=0 \quad \forall \chi \in L_{0}^{2}(\Omega) .
$$

This iteration scheme has the advantage that the resulting linear equations for the velocity field and the magnetic field decouple. Moreover, for the magnetic field the equations for the components of this field also uncouple. In this case, the computation reduces to solving several much smaller systems of linear equations. The disadvantage of this iterative scheme is that it converges only for initial guesses that are sufficiently close to the solution and that it requires some additional constraints on the data. In addition, convergence is slower, in general, than that of the first scheme. 
A third iterative scheme is Newton's method, i.e., we find

$$
\begin{aligned}
(\mathbf{u}, \mathbf{B})_{m+1} \in\left\{\mathbf{w} \in \mathbf{H}^{1}(\Omega)\right. & \left.:\left.\mathbf{w}\right|_{\partial \Omega}=\mathbf{g}\right\} \times\left\{\boldsymbol{\Phi} \in \mathbf{H}^{1}(\Omega):\left.(\boldsymbol{\Phi} \cdot \mathbf{n})\right|_{\partial \Omega}=q\right\} \\
& \text { and } p_{m+1} \in L_{0}^{2}(\Omega)
\end{aligned}
$$

such that

$$
\begin{aligned}
& a_{0}\left((\mathbf{u}, \mathbf{B})_{m+1},(\mathbf{v}, \Psi)\right)+\tilde{a}_{1}\left((\mathbf{u}, \mathbf{B})_{m},(\mathbf{u}, \mathbf{B})_{m+1},(\mathbf{v}, \Psi)\right) \\
& \quad+b\left((\mathbf{v}, \Psi), p_{m+1}\right)+\tilde{a}_{1}\left((\mathbf{u}, \mathbf{B})_{m+1},(\mathbf{u}, \mathbf{B})_{m},(\mathbf{v}, \Psi)\right) \\
& =F((\mathbf{v}, \Psi))+\tilde{a}_{1}\left((\mathbf{u}, \mathbf{B})_{m},(\mathbf{u}, \mathbf{B})_{m},(\mathbf{v}, \Psi)\right) \quad \forall(\mathbf{v}, \Psi) \in \mathscr{W}_{0 n}(\Omega)
\end{aligned}
$$

and

$$
b\left((\mathbf{u}, \mathbf{B})_{m+1}, \chi\right)=0 \quad \forall \chi \in L_{0}^{2}(\Omega) .
$$

The advantage of Newton's method is its guaranteed local and quadratic convergence, at least when uniqueness can be guaranteed. On the other hand, it converges only for sufficiently accurate initial guesses.

With regard to the three proposed schemes, we have the following results. The first and third of these follow by what are now standard arguments; see, e.g., [6,7], or [12]. Note that the assumption (7.10) is more restrictive than is the condition (4.26).

Proposition 7.1. If the assumptions of Theorem 4.6 and condition (4.26) hold, then the iteration scheme (7.1)-(7.3) is well defined and converges for any initial iterate $(\mathbf{u}, \mathbf{B})_{0} \in \mathscr{W}(\boldsymbol{\Omega})$.

Proposition 7.2. The iteration scheme (7.4)-(7.6) is well defined and converges provided the assumptions of Theorem 4.6 hold and the initial iterate $(\mathbf{u}, \mathbf{B})_{0} \in$ $\mathscr{W}(\Omega)$ satisfies

$$
\begin{aligned}
\frac{1}{\alpha}\left[\|\mathbf{f}\|_{-1}\right. & +\frac{1}{R_{m}}\|\mathbf{k}\|_{-1 / 2, \partial \Omega}+\gamma_{1} \gamma_{3}\left(\frac{\gamma_{1}}{N}+\frac{\gamma_{2}}{\sqrt{2} R_{m}}\right)\|\mathbf{g}\|_{1 / 2, \partial \Omega}^{2} \\
& \left.+\frac{\gamma_{1} \gamma_{2} \gamma_{3}}{\sqrt{2} R_{m}}\|q\|_{1 / 2, \partial \Omega}^{2}\right] \\
+ & \gamma_{1}\left(1+\frac{1}{\alpha M^{2}}\right)\|\mathbf{g}\|_{1 / 2, \partial \Omega}+\gamma_{2}\left(1+\frac{5}{\alpha R_{m}^{2}}\right)\|q\|_{1 / 2, \partial \Omega} \\
+ & \frac{1}{2}\left\|(\mathbf{u}, \mathbf{B})-(\mathbf{u}, \mathbf{B})_{0}\right\|_{\mathscr{W}} \\
< & \frac{1}{2} \min \left\{\frac{k_{1}}{M^{2}}, \frac{k_{2}}{R_{m}^{2}}\right\} / \sqrt{2} \gamma_{3} \max \left\{\frac{1}{N}, \frac{\sqrt{2}}{R_{m}}\right\} .
\end{aligned}
$$


Proof. One easily finds that

$$
\begin{aligned}
a_{0}\left((\mathbf{u}, \mathbf{B})-(\mathbf{u}, \mathbf{B})_{m+1},(\mathbf{u}, \mathbf{B})-(\mathbf{u}, \mathbf{B})_{m+1}\right) \\
=\tilde{a}_{1}\left((\mathbf{u}, \mathbf{B})-(\mathbf{u}, \mathbf{B})_{m},(\mathbf{u}, \mathbf{B})-(\mathbf{u}, \mathbf{B})_{m},(\mathbf{u}, \mathbf{B})_{\left.-(\mathbf{u}, \mathbf{B})_{m+1}\right)}\right) \\
\quad-\tilde{a}_{1}\left((\mathbf{u}, \mathbf{B}),(\mathbf{u}, \mathbf{B})-(\mathbf{u}, \mathbf{B})_{m},(\mathbf{u}, \mathbf{B})-(\mathbf{u}, \mathbf{B})_{m+1}\right) \\
\quad-\tilde{a}_{1}\left((\mathbf{u}, \mathbf{B})-(\mathbf{u}, \mathbf{B})_{m},(\mathbf{u}, \mathbf{B}),(\mathbf{u}, \mathbf{B})-(\mathbf{u}, \mathbf{B})_{m+1}\right) .
\end{aligned}
$$

Using (4.6) and (4.10), we get

$$
\begin{aligned}
\|(\mathbf{u}, \mathbf{B}) & -(\mathbf{u}, \mathbf{B})_{m+1} \|_{\mathscr{W}} \\
\leq & \sqrt{2} \gamma_{3} \max \left\{\frac{1}{N}, \frac{\sqrt{2}}{R_{m}}\right\} / \min \left\{\frac{k_{1}}{M^{2}}, \frac{k_{2}}{R_{m}^{2}}\right\} \\
\cdot & {\left[2\left\|(\mathbf{u}, \mathbf{B})-(\mathbf{u}, \mathbf{B})_{m}\right\|_{\mathscr{W}}\|(\mathbf{u}, \mathbf{B})\|_{\mathscr{W}}+\left\|(\mathbf{u}, \mathbf{B})-(\mathbf{u}, \mathbf{B})_{m}\right\|_{\mathscr{W}}^{2}\right], }
\end{aligned}
$$

and using the a priori estimate (4.22), we obtain

$$
\begin{aligned}
\|(\mathbf{u}, \mathbf{B}) & -(\mathbf{u}, \mathbf{B})_{m+1} \|_{\mathscr{W}} \\
\leq & \sqrt{2} \gamma_{3} \max \left\{\frac{1}{N}, \frac{\sqrt{2}}{R_{m}}\right\} / \min \left\{\frac{k_{1}}{M^{2}}, \frac{k_{2}}{R_{m}^{2}}\right\} \\
& {\left[2 \left[\frac { 1 } { \alpha } \left(\|\mathbf{f}\|_{-1}+\frac{1}{R_{m}}\|\mathbf{k}\|_{-1 / 2, \partial \Omega}+\gamma_{1} \gamma_{3}\left(\frac{\gamma_{1}}{N}+\frac{\gamma_{2}}{\sqrt{2} R_{m}}\right)\|\mathbf{g}\|_{1 / 2, \partial \Omega}^{2}\right.\right.\right.} \\
& \left.+\frac{\gamma_{1} \gamma_{2} \gamma_{3}}{\sqrt{2} R_{m}}\|q\|_{1 / 2, \partial \Omega}^{2}\right) \\
\left.+\gamma_{1}\left(1+\frac{1}{\alpha M^{2}}\right)\|\mathbf{g}\|_{1 / 2, \partial \Omega}+\gamma_{2}\left(1+\frac{5}{\alpha R_{m}^{2}}\right)\|q\|_{1 / 2, \partial \Omega}\right] & \left.+\left\|(\mathbf{u}, \mathbf{B})-(\mathbf{u}, \mathbf{B})_{m}\right\|_{\mathscr{W}}\right]\left\|(\mathbf{u}, \mathbf{B})-(\mathbf{u}, \mathbf{B})_{m}\right\|_{\mathscr{W}} .
\end{aligned}
$$

Finally, using condition (7.10), we have that

$$
\left\|(\mathbf{u}, \mathbf{B})-(\mathbf{u}, \mathbf{B})_{m+1}\right\|_{\mathscr{W}} \leq c\left\|(\mathbf{u}, \mathbf{B})-(\mathbf{u}, \mathbf{B})_{m}\right\|_{\mathscr{W}},
$$

for some constant $c<1$, which gives the required result.

Proposition 7.3. If the assumptions of Theorem 4.6 and condition (4.26) hold, then equations (7.7)-(7.9) have a solution and the iterative scheme defined by them converges quadratically, provided the initial iterate $(\mathbf{u}, \mathbf{B})_{0} \in \mathscr{W}(\Omega)$ 
satisfies

$$
\begin{gathered}
\left\|(\mathbf{u}, \mathbf{B})-(\mathbf{u}, \mathbf{B})_{0}\right\|_{\mathscr{W}} \\
\leq c\left[\min \left\{\frac{k_{1}}{M^{2}}, \frac{k_{2}}{R_{m}^{2}}\right\} / \sqrt{2} \gamma_{3} \max \left\{\frac{1}{N}, \frac{\sqrt{2}}{R_{m}}\right\}\right. \\
-\left[\frac { 1 } { \alpha } \left(\|\mathbf{f}\|_{-1}+\frac{1}{R_{m}}\|\mathbf{k}\|_{-1 / 2, \partial \Omega}+\gamma_{1} \gamma_{3}\left(\frac{\gamma_{1}}{N}+\frac{\gamma_{2}}{\sqrt{2} R_{m}}\right)\|\mathbf{g}\|_{1 / 2, \partial \Omega}^{2}\right.\right. \\
\left.+\frac{\gamma_{1} \gamma_{2} \gamma_{3}}{\sqrt{2} R_{m}}\|q\|_{1 / 2, \partial \Omega}^{2}\right) \\
\left.\left.+\gamma_{1}\left(1+\frac{1}{\alpha M^{2}}\right)\|\mathbf{g}\|_{1 / 2, \partial \Omega}+\gamma_{2}\left(1+\frac{5}{\alpha R_{m}^{2}}\right)\|q\|_{1 / 2, \partial \Omega}\right]\right]
\end{gathered}
$$

for some constant $c<1 / 2$.

\section{BIBLIOGRAPHY}

1. R. A. Adams, Sobolev spaces, Academic Press, New York, 1975.

2. E. V. Chizhonkov, On a system of equations of magnetohydrodynamic type, Soviet Math. Dokl. 30 (1984), 542-545.

3. P. Ciarlet, The finite element method for elliptic problems, North-Holland, 1977.

4. G. Duvaut and J. L. Lions, Inequalities in mechanics and physics, Springer-Verlag, New York, 1976.

5. G. Fix, M. D. Gunzburger, and J. S. Peterson, On finite element approximations of problems having inhomogeneous essential boundary conditions, Comput. Math. Appl. 9 (1983), 687700 .

6. V. Girault and P.-A. Raviart, Finite element approximation of the Navier-Stokes equations, Lecture Notes in Math., vol. 749, Springer-Verlag, New York, 1981.

7. _ Finite element methods for Navier-Stokes equations. Theory and algorithms, SpringerVerlag, New York, 1986.

8. P. Grisvard, Elliptic problems in nonsmooth domains, Pitman, Boston, 1985.

9. M. D. Gunzburger and J. S. Peterson, On conforming finite element methods for the inhomogeneous stationary Navier-Stokes equations, Numer. Math. 42 (1983), 173-194.

10. W. F. Hughes and F. J. Young, The electromagnetodynamics of fluids, Wiley, New York, 1966.

11. J. D. Jackson, Classical electrodynamics, Wiley, New York, 1975.

12. O. A. Karakashian, On a Galerkin-Lagrange multiplier method for the stationary NavierStokes equations, SIAM J. Numer. Anal. 19 (1982), 909-923.

13. R. A. Nicolaides, Existence, uniqueness and approximation for generalized saddle point problems, SIAM J. Numer. Anal. 19 (1982), 349-357.

14. J. S. Peterson, On the finite element approximation of incompressible flows of an electrically conducting fluid, Numer. Methods Partial Differential Equations 4 (1988), 57-68.

15. J. A. Shercliff, A textbook of magnetohydrodynamics, Pergamon Press, Oxford, 1965.

16. R. Stenberg, On some three-dimensional finite elements for incompressible media, Comput. Methods Appl. Mech. Engrg. 63 (1987), 261-269.

17. R. Temam, Navier Stokes equations. Theory and numerical analysis, North-Holland, Amsterdam, 1984.

18. J. S. Walker, Large interaction parameter magnetohydrodynamics and applications in fusion reactor technology, Fluid Mechanics in Energy Conversion (J. Buckmaster, ed.), SIAM, Philadelphia, 1980. 
19. J. S. Walker, Three dimensional MHD flows in rectangular ducts with thin conducting walls and strong transverse nonuniform magnetic fields, Liquid-Metal Flows and Magnetohydrodynamics (H. Branover, P. S. Lykoudis, and A. Yakhot, eds.), AIAA, New York, 1983.

20. N. S. Winowich, Magnetofluidynamic channel flow with a nonuniform magnetic field and conductive walls, Ph. D. thesis, Carnegie Mellon University, Pittsburgh, 1986.

21. N. S. Winowich and W. Hughes, A finite element analysis of two dimensional mhd flow, Liquid-Metal Flows and Magnetohydrodynamics (H. Branover, P. S. Lykoudis, and A. Yakhot, eds.), AIAA, New York, 1983.

Department of Mathematics, Carnegie Mellon University, Pittsburgh, Pennsylvania 15213

Computing and Communications Division, Los Alamos National Laboratory, Los AlaMOS, New MeXico 87545

Current address, M. D. Gunzburger and J. S. Peterson: Department of Mathematics, Virginia Polytechnic Institute \& State University, Blacksburg, Virginia 24061

E-mail address: gunzburger@vtcc1.bitnet

E-mail adress, peterson@vtccl.bitnet

Current address, A. J. Meir: Department of Foundations, Analysis, and Topology, Auburn University, Auburn, Alabama 36849

E-mail address: ameir@auducvax.bitnet 